\title{
$Z$ mediated flavor changing neutral currents with a fourth vectorlike family
}

\author{
A. E. Cárcamo Hernández@ ${ }^{1,2,3, *}$ S. F. King, ${ }^{4, \uparrow}$ and H. Lee $\oplus^{4, \$}$ \\ ${ }^{1}$ Departamento de Física, Universidad Técnica Federico Santa María, Casilla 110-V, Valparaíso, Chile \\ ${ }^{2}$ Centro Científico-Tecnológico de Valparaíso, Casilla 110-V, Valparaíso, Chile \\ ${ }^{3}$ Millennium Institute for Subatomic Physics at High-Energy Frontier (SAPHIR), \\ Fernández Concha 700, Santiago, Chile \\ ${ }^{4}$ School of Physics and Astronomy, University of Southampton, SO17 1BJ Southampton, United Kingdom
}

(Received 28 October 2021; accepted 3 January 2022; published 20 January 2022)

\begin{abstract}
We discuss $Z$ mediated flavor changing neutral currents within a model where the hierarchical quark and lepton masses are explained via a fourth vectorlike family, together with a scalar sector consisting of two Higgs doublets augmented by a gauge singlet scalar field that spontaneously breaks an extra global $U(1)^{\prime}$ symmetry. The $Z$ mediated flavor violating interactions arise from the mixings between the SM fermions and the vectorlike fermions, where the mixing is discussed in an analytic approximation and also exactly numerically. We first discuss charged lepton flavor violating (CLFV) $\tau \rightarrow \mu \gamma, \tau \rightarrow 3 \mu$ and $Z \rightarrow \mu \tau$ decays and find that they cannot significantly constrain the masses of charged vectorlike leptons. However, the $790 \mathrm{GeV}$ mass bound arising from collider searches on vectorlike lepton doublets can set further constraints on the model parameter space. We also consider rare $t \rightarrow c Z$ decays as well as unitarity violation in the CKM mixing in order to constrain the quark sector of the model under consideration.
\end{abstract}

DOI: 10.1103/PhysRevD.105.015021

\section{INTRODUCTION}

A great success of the energy frontier is the discovery of the Higgs particle by ATLAS and CMS collaborations at the Large Hadron Collider (LHC) on 4th July 2012 [1,2]. After that discovery, no new particle has been found so far by the experiments at LHC with $13 \mathrm{TeV}$ proton-proton center of mass energy. This highlights the fact that not just the energy frontier but also the luminosity (intensity) frontier should be considered as of equal importance in the search for physics beyond the Standard Model (SM). For example, one may consider observables mediated by flavor-changing neutral currents (FCNCs), which are quite sensitive to new physics, since such FCNC observables are extremely suppressed in the SM due to the well-known Glashow-Illiopoulos-Maiani (GIM) mechanism. Another example of a highly suppressed process is provided by the branching ratio of $\mu \rightarrow e \gamma$ decay mediated by massive neutrinos at the one-loop level [3]:

$$
\mathrm{BR}(\mu \rightarrow e \gamma) \approx 10^{-55}
$$

\footnotetext{
*antonio.carcamo@usm.cl

†ing@soton.ac.uk

"hl2n18@soton.ac.uk
}

Published by the American Physical Society under the terms of the Creative Commons Attribution 4.0 International license. Further distribution of this work must maintain attribution to the author(s) and the published article's title, journal citation, and DOI. Funded by SCOAP.
The experimentally known sensitivity for the branching ratio of $\mu \rightarrow e \gamma$ is

$$
\mathrm{BR}(\mu \rightarrow e \gamma)_{\mathrm{EXP}}=4.2 \times 10^{-13}
$$

The large gap between the tiny rates of the flavor violating decays predicted by the SM and their experimental upper limits has motivated the construction of many flavor models with extended scalar, quark and leptonic spectrum aimed at enhancing those rates by several orders of magnitude up to an observable level within the reach of the sensitivity of the future experiments. A similar situation occurs for other rare FCNC decays such as, for instance $Z \rightarrow \mu \tau$ and $t \rightarrow c Z$, which are very suppressed in the SM, but in extensions of the SM, can acquire sizeable values, within the reach of the future experimental sensitivity. Although various models with a heavy $Z^{\prime}$ boson have also received a lot of attention by the particle physics community as a new source of FCNCs, its properties, being not fully constrained, do not lead to definite predictions. For this reason we shall restrict ourselves to the SM $Z$ couplings in this paper.

In this paper we focus on the SM Z FCNC interactions induced by tree-level gauge boson exchange in a model in which the fermion sector of the SM is enlarged with a fourth vectorlike family. An interesting feature of this approach is all coupling constants of $Z$ interactions in this work are fixed by the known values of the SM $Z$ gauge boson interactions, together with mixing parameters. 
Our main motivation for adding a fourth vectorlike family is to explain quark and lepton mass hierarchies. We first forbid the SM Yukawa couplings with a global $U(1)^{\prime}$ symmetry, then allow them to be generated effectively via mixing with the fourth vectorlike family, a mechanism somewhat analogous to the seesaw mechanism for neutrino masses. Consequently, the SM charged fermion masses are inversely proportional to the masses of the heavy vectorlike leptons and directly proportional to the product of the couplings of Yukawa interactions that mix SM charged fermions with vectorlike fermions. This implies that a small hierarchy in those couplings can yield a quadratically larger hierarchy in the effective couplings. Combined with a moderate hierarchy in the vectorlike masses, this allows us to naturally explain the SM charged fermion mass hierarchy and to predict the mass scale of vectorlike fermions.

A similar model was discussed in our previous works [4], although with two vectorlike families, but the effect on the $Z$ and $W$ boson couplings was not studied. In our previous work [4], whose purpose was to explain the muon and electron anomalous magnetic moments simultaneously, the main focus was on the 2HDM scalar sector, and the FCNCs arising from the $Z$ and $W$ boson couplings were not considered, since the full mass matrices were not accurately diagonalized, and hence such effects were beyond the approximations used there. By contrast, the main goal of this work is to study the $\mathrm{SM} Z$ and $W$ contributions to the FCNC observables at leading order to constrain the masses of vectorlike fermions, and to explore other possible phenomenological signatures. The SM $W$ contributions to the CKM mixing matrix with the extended quark sector are also studied for the first time in this work. In order for these effects to be considered reliably, the mass matrices of each fermion sector are accurately diagonalized, both numerically and analytically, unlike the previous work where simple approximations were used which masked the effects we consider here. The results in this work are sufficiently accurate to enable the contribution of the $Z$ and $W$ boson couplings to physics beyond the SM to be reliably considered for the first time.

In order to make the results completely transparent, we shall study the $Z$ and $W$ boson couplings in the presence of only one vectorlike family so that mass matrices of this work can be straightforwardly diagonalized using both analytical and numerical methods. Since only one vectorlike fermion family is used, the first generation of SM charged fermions do not acquire masses, which nonetheless is a very good approximation considering the SM fermions belonging to the first family are very light. Consequently, we restrict our attention to the second and third generations of SM fermions, as well as to several observables related to FCNC processes involving the second and third SM families. In our approach, then, the SM is a low effective energy theory arising after integrating out a single heavy fourth vectorlike family. In order to dynamically generate the hierarchical structure of SM fermion masses, the fermionic mass matrices given in [4] as well as the ones obtained in this work must be accurately and completely diagonalized, which, as mentioned above, has not been done previously. The mass matrices for the charged lepton and up-quark sectors share the same structure, whereas the one for the down-type quark sector involves an additional nonzero element in a particular basis, although we later show that the results are basis independent. This reasoning does not apply to the neutrino sector, since this sector is treated independently. This different feature of the downtype mass matrix, in the preferred basis, allows us to achieve all mixings among the three generations of SM fermions even though the first one remains massless, and this leads to a prediction for the Cabbibo-KobayashiMaskawa (CKM) mixing matrix. In addition, due to the mixings between the SM quarks and the vectorlike quarks, the CKM quark mixing matrix originating from the $W$ couplings is not unitary, thus implying the need of relaxing the unitarity condition of the CKM mixing matrix, and we also study this feature.

This paper is organized as follows. In Sec. II we introduce our model to explain the origin of the SM fermion's mass with a fourth vectorlike family. In Sec. III the mass matrices in both quark and lepton sectors are constructed and diagonalized using the mixing formalism. In Sec. IV the $Z$ gauge boson interactions with fermions are determined from the mixing matrices used in the mass matrix diagonalization. Several FCNC observables for both lepton and quark sectors are analyzed in detail in Secs. V and VI, respectively. We state our conclusions in Sec. VII. Several technical details are relegated to the Appendixes. The perturbative analytical diagonalization of the mass matrices for the charged lepton, up type quark and down type quark sectors are discussed in detail in Appendixes A, B, and C, respectively. The comparison between the numerical and approximate analytic diagonalization of the mass matrices for charged leptons and quarks is made in Appendixes D and E, respectively.

\section{AN EXTENDED MODEL WITH A FOURTH VECTORLIKE FAMILY}

The origin of the pattern of SM fermion masses is interesting open question, not addressed by the SM. The mass parameters of the SM have been experimentally determined with good precision, and these experimentally observed mass parameters show a strong hierarchical structure of the SM fermion masses. The most extreme hierarchy is exhibited between the SM neutrino Yukawa coupling of about $10^{-12}$ and the top quark Yukawa coupling of about 1 . Regarding the tiny neutrino masses, many particle physicists regard their masses as most likely explained by the see-saw mechanism rather than by the Yukawa interactions, thus predicting the presence of the heavy right-handed neutrinos. The reason why the see-saw 
TABLE I. Particle assignments under the $S U(3)_{C} \times S U(2)_{L} \times U(1)_{Y} \times U(1)^{\prime}$ symmetry of the extended $2 \mathrm{HDM}$ theory with fourth vectorlike family. The index $i=1,2,3$ denotes the $i$ th SM fermion generation and $k=4$ stands for the fourth vectorlike family.

\begin{tabular}{lcccccccccccccccccccc}
\hline \hline Field & $Q_{i L}$ & $u_{i R}$ & $d_{i R}$ & $L_{i L}$ & $e_{i R}$ & $Q_{k L}$ & $u_{k R}$ & $d_{k R}$ & $L_{k L}$ & $e_{k R}$ & $\nu_{k R}$ & $\tilde{Q}_{k R}$ & $\tilde{u}_{k L}$ & $\tilde{d}_{k L}$ & $\tilde{L}_{k R}$ & $\tilde{e}_{k L}$ & $\tilde{\nu}_{k R}$ & $\phi$ & $H_{u}$ & $H_{d}$ \\
\hline$S U(3)_{C}$ & $\mathbf{3}$ & $\mathbf{3}$ & $\mathbf{3}$ & $\mathbf{1}$ & $\mathbf{1}$ & $\mathbf{3}$ & $\mathbf{3}$ & $\mathbf{3}$ & $\mathbf{1}$ & $\mathbf{1}$ & $\mathbf{1}$ & $\mathbf{3}$ & $\mathbf{3}$ & $\mathbf{3}$ & $\mathbf{1}$ & $\mathbf{1}$ & $\mathbf{1}$ & $\mathbf{1}$ & $\mathbf{1}$ & $\mathbf{1}$ \\
$S U(2)_{L}$ & $\mathbf{2}$ & $\mathbf{1}$ & $\mathbf{1}$ & $\mathbf{2}$ & $\mathbf{1}$ & $\mathbf{2}$ & $\mathbf{1}$ & $\mathbf{1}$ & $\mathbf{2}$ & $\mathbf{1}$ & $\mathbf{1}$ & $\mathbf{2}$ & $\mathbf{1}$ & $\mathbf{1}$ & $\mathbf{2}$ & $\mathbf{1}$ & $\mathbf{1}$ & $\mathbf{1}$ & $\mathbf{2}$ & $\mathbf{2}$ \\
$U(1)_{Y}$ & $\frac{1}{6}$ & $\frac{2}{3}$ & $-\frac{1}{3}$ & $-\frac{1}{2}$ & 1 & $\frac{1}{6}$ & $\frac{2}{3}$ & $-\frac{1}{3}$ & $-\frac{1}{2}$ & -1 & 0 & $\frac{1}{6}$ & $\frac{2}{3}$ & $-\frac{1}{3}$ & $-\frac{1}{2}$ & -1 & 0 & 0 & $\frac{1}{2}$ & $-\frac{1}{2}$ \\
$U(1)^{\prime}$ & 0 & 0 & 0 & 0 & 0 & 1 & -1 & -1 & 1 & -1 & -1 & 1 & -1 & -1 & 1 & -1 & -1 & 1 & -1 & -1 \\
\hline \hline
\end{tabular}

mechanism has received a large amount of attention by the particle physics community is that it provides a dynamical explanation of the tiny active neutrino masses. For a similar reason, it is interesting to speculate about the existence of a dynamical mechanism that produces the masses of all SM fermions via the exchange of heavy fermionic degrees of freedom thus implying that the SM is an effective low energy theory arising from some spontaneous breaking at higher energy scales of a more complete underlying theory. In order to specify a possible candidate of an underlying theory responsible for the generation of the SM fermion mass hierarchy, we shall consider a minimal extension of the SM consistent with the SM current experimental bounds. With this motivation in mind, we enlarge the SM fermion and scalar sectors by including a fourth vectorlike family and an extra $S U(2)$ scalar doublet as well as a scalar singlet, respectively. Furthermore, we extend the SM gauge symmetry by adding a $U(1)^{\prime}$ global symmetry. The particle content of the proposed model is shown in Table I.

Our proposed theory is a minimal extended 2 Higgs doublet model (2HDM) where the SM fermion sector is enlarged by the inclusion of a fourth vectorlike family and the scalar sector is augmented by an extra $S U(2)$ scalar doublet and a singlet flavon and lastly the SM gauge symmetry is extended by the $U(1)^{\prime}$ global symmetry. As this model features the global $U(1)^{\prime}$ symmetry, there is no a neutral $Z^{\prime}$ gauge boson in the particle spectrum. Furthermore, the up-type quarks feature Yukawa interactions with the up-type SM Higgs $H_{u}$, whereas the extra scalar doublet $H_{d}$ couples with the SM down-type quarks and charged leptons. Our proposed model is especially motivated by the hierarchical structure of the SM and, in order to implement this hierarchy, we forbid the SM-type Yukawa interactions by appropriate $U(1)^{\prime}$ charge assignments of the scalar and fermionic fields. Then, for the above specified particle content, the following effective Yukawa interactions arise:

$$
\begin{aligned}
\mathcal{L}_{\text {eff }}^{\text {Yukawa }}= & y_{i k}^{\psi}\left(M_{\psi^{\prime}}^{-1}\right)_{k l} x_{l j}^{\psi^{\prime}}\langle\phi\rangle \bar{\psi}_{i L} \tilde{H} \psi_{j R} \\
& +x_{i k}^{\psi^{\prime}}\langle\phi\rangle\left(M_{\psi}^{-1}\right)_{k l} y_{l j}^{\psi} \bar{\psi}_{i L} \tilde{H} \psi_{j R}+\text { H.c. }
\end{aligned}
$$

where the indices $i, j=1,2,3$ and $k, l=4$ whereas $\psi, \psi^{\prime}=Q, u, d, L, e$ and $M$ means heavy vectorlike mass. The masses of all SM fermions can be explained by this effective Lagrangian of Eq. (3), emphasizing their relative different masses are explained by the factor $\langle\phi\rangle / M \ll 1$ (apart from top quark), except for the neutrinos which requires an independent approach to their mass. Feynman diagrams corresponding for the effective Lagrangian are shown in Fig. 1:

The theory considered in this paper corresponds to the one given in one of our previous works [4], however one vectorlike family is used instead of two so that the mass matrices for both quark and lepton sectors can be diagonalized much more economically than in our previous model of [4] at cost of having massless the first generation SM charged fermions (One of our main purposes is to diagonalize mass matrices for the quark and lepton sectors without any assumptions) and this is actually a good approximation taking into account that the first generation of SM charged fermions are very light.
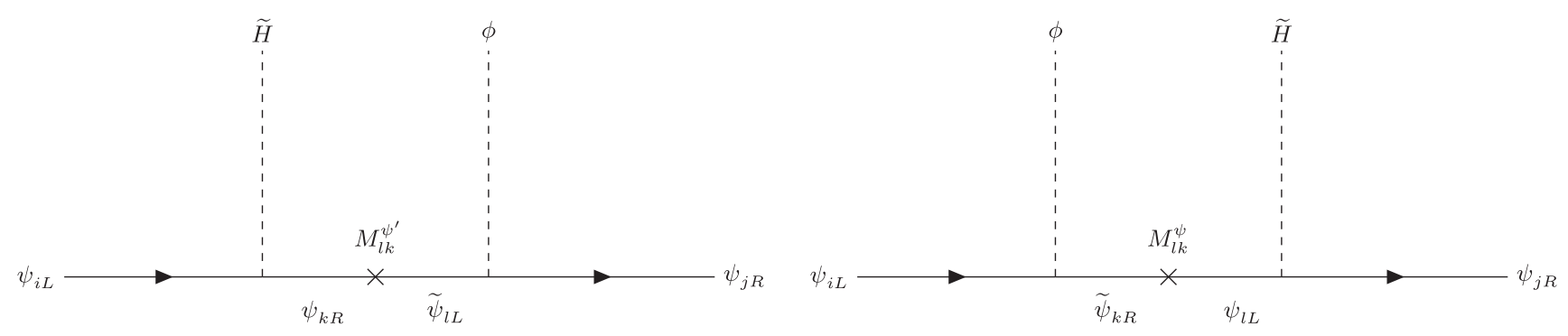

FIG. 1. Feynman diagrams leading to the effective Yukawa interactions, where $\psi, \psi^{\prime}=Q, u, d, L, e$ (neutrinos will be treated separately), $i, j=1,2,3, k, l=4, M_{l k}$ is vectorlike mass and $\tilde{H}=i \sigma_{2} H^{*}, H=H_{u, d}$. 

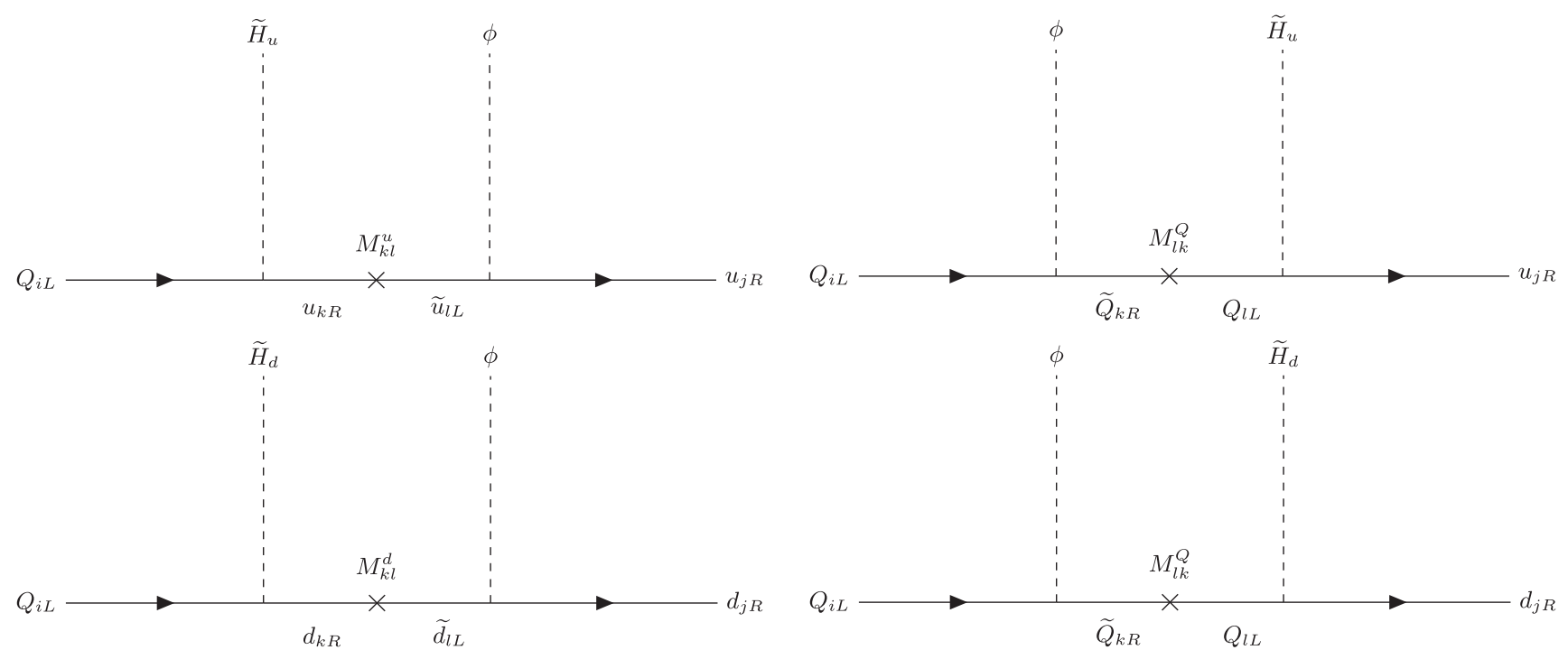

FIG. 2. Feynman diagrams contributing to the up and down type quark's effective Yukawa interactions in the mass insertion formalism. Here $i, j=1,2,3$ and $k, l=4$ and $M_{l k}$ is vectorlike mass.

\section{A. Effective Yukawa interactions for the SM fermions}

The renormalizable interactions of the quark sector in this model are given by:

$$
\begin{aligned}
\mathcal{L}_{q}^{\text {Yukawa }+ \text { Mass }}= & y_{i k}^{u} \bar{Q}_{i L} \tilde{H}_{u} u_{k R}+x_{k i}^{u} \phi \overline{\tilde{u}}_{k L} u_{i R}+x_{i k}^{Q} \phi \bar{Q}_{i L} \tilde{Q}_{k R} \\
& +y_{k i}^{u} \bar{Q}_{k L} \tilde{H}_{u} u_{i R}+y_{i k}^{d} \bar{Q}_{i L} \tilde{H}_{d} d_{k R} \\
& +x_{k i}^{d} \phi \overline{\tilde{d}}_{k L} d_{i R}+y_{k i}^{d} \bar{Q}_{k L} \tilde{H}_{d} d_{i R} \\
& +M_{k l}^{u} \overline{\tilde{u}}_{l L} u_{k R}+M_{k l}^{d} \overline{\tilde{d}}_{l L} d_{k R} \\
& +M_{k l}^{Q} \bar{Q}_{k L} \tilde{Q}_{l R}+\text { H.c. }
\end{aligned}
$$

where $i, j=1,2,3, k, l=4$ and $\tilde{H}=i \sigma_{2} H^{*}$. After the $U(1)^{\prime}$ symmetry is spontaneously broken by the vacuum expectation value (vev) of the singlet flavon $\phi$, and the heavy vectorlike fermions are integrated out, the renormalizable Yukawa terms at higher energy scale give rise to the effective Yukawa interactions which explain the current SM fermion mass hierarchy. The Feynman diagrams corresponding to the effective Yukawa interactions of the quark sector are shown in Fig. 2:

The same approach can be applied to the SM charged lepton sector and the renormalizable charged lepton Yukawa interactions are given by:

$$
\begin{aligned}
\mathcal{L}_{e}^{\text {Yukawa }+ \text { Mass }}= & y_{i k}^{e} \bar{L}_{i L} \tilde{H}_{d} e_{k R}+x_{k i}^{e} \phi \overline{\tilde{e}}_{k L} e_{i R} \\
& +x_{i k}^{L} \phi \bar{L}_{i L} \tilde{L}_{k R}+y_{k i}^{e} \bar{L}_{k L} \tilde{H}_{d} e_{i R} \\
& +M_{k l}^{e} \overline{\tilde{e}}_{l L} e_{k R}+M_{k l}^{L} \bar{L}_{k L} \tilde{L}_{l R}+\text { H.c., }
\end{aligned}
$$

and its following effective Yukawa interactions read off in Fig. 3.

It is possible to generate the masses of all SM charged fermions by the same method relying on effective Yukawa interactions. However, this is not the case for the SM light active neutrinos as they need to be independently treated since the simplest mechanism responsible for generating their tiny masses requires the inclusion of Majorana particles in the leptonic spectrum. In order to make the SM neutrinos massive, we made use of two important
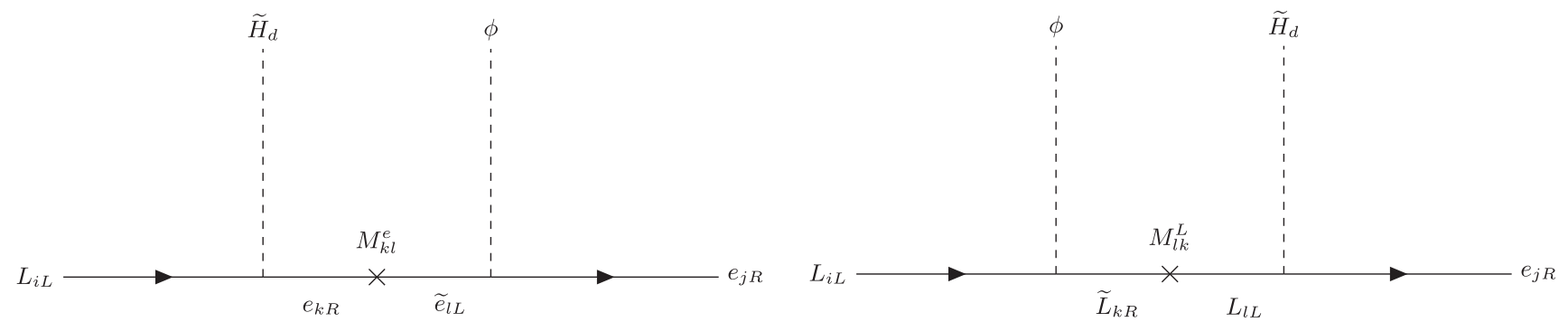

FIG. 3. Feynman diagrams contributing to the charged lepton's effective Yukawa interactions in the mass insertion formalism. Here $i$, $j=1,2,3$ and $k, l=4$ and $M_{l k}$ is vectorlike mass. 


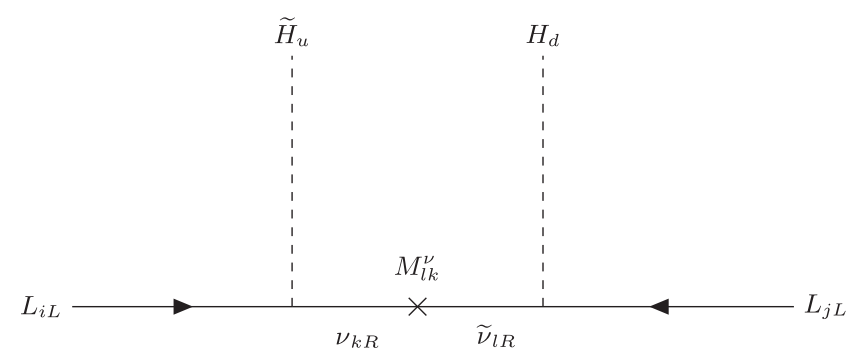

FIG. 4. Type Ib seesaw diagram [4,5] which leads to the effective Yukawa interactions for the Majorana neutrinos in mass insertion formalism, where $i, j=1,2,3$ and $k, l=4$ and $M_{l k}$ is vectorlike mass.

assumptions, one of which is that the SM neutrinos are Majorana particles and the other is they get masses via the type $1 \mathrm{~b}$ seesaw mechanism [4,5] mediated by the heavy vectorlike neutrinos without considering the right-handed neutrinos $\nu_{i R}$. The renormalizable Yukawa interactions for the neutrino sector are given by:

$$
\begin{aligned}
\mathcal{L}_{\nu}^{\text {Yukawa }+ \text { Mass }}= & y_{i k}^{\nu} \bar{L}_{i L} \tilde{H}_{u} \nu_{k R}+x_{i k}^{L} \bar{L}_{i L} H_{d} \overline{\tilde{\nu}}_{k R} \\
& +M_{k l}^{M} \overline{\tilde{\nu}}_{l R} \nu_{k R}+\text { H.c. }
\end{aligned}
$$

It is worth mentioning that the nature of the vectorlike mass appearing in Eq. (6) is that the vectorlike mass is different than the Majorana mass since the particles involved in vectorlike mass terms are different, whereas the ones appearing in a Majorana mass terms does not. However, they share the common feature that both break the lepton number, which is confirmed by checking each lepton number of $\nu_{k R}$ and $\tilde{\nu}_{k R}$ in the two Yukawa interactions of Eq. (6). We call this mechanism "type $1 \mathrm{~b}$ seesaw mechanism" and it can allow a different Yukawa interaction at each vertex as seen in Fig. 4.

Allowing a different Yukawa interaction at each vertex of Fig. 4 means that one of the Yukawa interactions can have a very suppressed coupling constant, which can lower the expected order of magnitude of the right-handed Majorana neutrinos masses of the usual type I seesaw mechanism from $10^{14} \mathrm{GeV}$ up to the $\mathrm{TeV}$ scale. The most relevant features of the model considered in this paper are

(1) It allows a dynamical and natural explanation of the origin of the observed SM fermion mass hierarchy.

(2) The model under consideration is economical in the sense that it includes a common mechanism for generating the masses of the SM charged fermions via effective Yukawa interactions resulting after integrating out the heavy vectorlike fermions.

(3) The expected right-handed neutrinos can have a much smaller mass compared to the ones mediating the usual type I seesaw mechanism, thus allowing to test our model at colliders as well as via charged lepton flavor violating processes.
Now that we have discussed how the SM fermions get massive via the effective Yukawa interactions, the next task is to construct their mass matrices in the flavor basis and then to diagonalize those and this will be discussed in the next section.

\section{EFFECTIVE YUKAWA MATRICES USING A MIXING FORMALISM}

The effective Yukawa interactions discussed in Sec. II give rise to the following mass matrix for fermions written in the flavor basis:

$$
M^{\psi}=\left(\begin{array}{c|ccccc} 
& \psi_{1 R} & \psi_{2 R} & \psi_{3 R} & \psi_{4 R} & \tilde{\psi}_{4 R} \\
\hline \bar{\psi}_{1 L} & 0 & 0 & 0 & y_{14}^{\psi}\left\langle\tilde{H}^{0}\right\rangle & x_{14}^{\psi}\langle\phi\rangle \\
\bar{\psi}_{2 L} & 0 & 0 & 0 & y_{24}^{\psi}\left\langle\tilde{H}^{0}\right\rangle & x_{24}^{\psi}\langle\phi\rangle \\
\bar{\psi}_{3 L} & 0 & 0 & 0 & y_{34}^{\psi \prime}\left\langle\tilde{H}^{0}\right\rangle & x_{34}^{\psi}\langle\phi\rangle \\
\bar{\psi}_{4 L} & y_{41}^{\psi}\left\langle\tilde{H}^{0}\right\rangle & y_{42}^{\psi}\left\langle\tilde{H}^{0}\right\rangle & y_{43}^{\psi}\left\langle\tilde{H}^{0}\right\rangle & 0 & M_{44}^{\psi} \\
\overline{\tilde{\psi}}_{4 L} & x_{41}^{\psi^{\prime}}\langle\phi\rangle & x_{42}^{\psi^{\prime}}\langle\phi\rangle & x_{43}^{\psi^{\prime}}\langle\phi\rangle & M_{44}^{\psi^{\prime}} & 0
\end{array}\right),
$$

where $\psi, \psi^{\prime}=Q, u, d, L, e$ and the zeros in the upper-left $3 \times 3$ block mean that the SM fermions acquire masses only via their mixing with the fourth vectorlike family. The other zeros appearing in the diagonal positions are forbidden by the $U(1)^{\prime}$ charge conservation. This mass matrix was obtained for the first time in [6] and it can reveal the hierarchical structure of the SM since this mass matrix involves three different mass scales $\left\langle H^{0}\right\rangle,\langle\phi\rangle$, and $M$. In order to dynamically reproduce the hierarchical structure of the SM fermion masses, we need to maximally rotate this mass matrix and the resulting maximally rotated mass matrix should be a starting point for our analysis in this work. For the fully rotated mass matrix, the up-quark and charged lepton sectors share the same structure, whereas the down-type quark mass matrix has an additional element since one of the quark doublet rotations was already used in the up quark sector, as it will be shown below. Regarding the diagonalization for each fermion sector, we will employ two methods for comparison; one of which is the numerical SVD diagonalization and the other is the analytical perturbative step-by-step diagonalization. We will make use of the numerical SVD diagonalization for the exact diagonalization as well as for our numerical scans in main body of this work, however it is important to look at the analytical approximated step-by-step diagonalization since it provides an analytical understanding on how the SM $Z$ gauge boson can induce the flavor violating interactions at tree-level and this analytic diagonalization will be covered in Appendix A to C. Lastly, we have found that the analytic diagonalization for each fermion sector is quite close to its numerical result with very small differences and this feature will be discussed in detail in Appendix D to E. 


\section{A. Diagonalizing the charged lepton mass matrix}

After all scalars of our proposed model acquire their vevs $\left(v_{d}=\left\langle H_{d}^{0}\right\rangle\right.$ and $\left.v_{\phi}=\langle\phi\rangle\right)$ from Eq. (7), we obtain the following fully rotated mass matrix for the charged lepton sector

$M^{e}=\left(\begin{array}{c|ccccc} & e_{1 R} & e_{2 R} & e_{3 R} & e_{4 R} & \tilde{L}_{4 R} \\ \hline \bar{L}_{1 L} & 0 & 0 & 0 & 0 & 0 \\ \bar{L}_{2 L} & 0 & 0 & 0 & y_{24}^{e} v_{d} & 0 \\ \bar{L}_{3 L} & 0 & 0 & 0 & y_{34}^{e} v_{d} & x_{34}^{L} v_{\phi} \\ \bar{L}_{4 L} & 0 & 0 & y_{43}^{e} v_{d} & 0 & M_{44}^{L} \\ \bar{e}_{4 L} & 0 & x_{42}^{e} v_{\phi} & x_{43}^{e} v_{\phi} & M_{44}^{e} & 0\end{array}\right)$

where we use this fully rotated basis as a starting point in order to easily explain the observed SM fermion mass hierarchy. This rotated basis is exactly consistent with the one given in [6] and we need to explain how the mass matrix of Eq. (8) is fully rotated. First of all, we rotate the left-handed leptonic fields $L_{1 L}$ and $L_{3 L}$ to turn off the entry $x_{14}^{L} v_{\phi}$ and then rotate $L_{2 L}$ and $L_{3 L}$ to turn off the next $x_{24}^{L} v_{\phi}$ entry. Next, we can rotate again the leptonic fields $L_{1 L}$ and $L_{2 L}$ to turn off the $y_{14}^{e} v_{d}$ entry. These rotations can be applied to the right-handed leptonic fields $e_{1,2,3 R}$ in order to make the zeros appearing in the lower-left $2 \times 3$ block. This fully rotated mass matrix of Eq. (8) is our starting point to implement both the hierarchical structure of the SM fermion masses and to analyze the flavor violating interactions mediated by the SM $Z$ gauge boson. Before diagonalizing the mass matrix of Eq. (8), it is convenient to rearrange the mass matrix by switching the Yukawa terms by mass parameters and then by swapping the fourth and fifth column in order to make the heavy vectorlike masses locate in the diagonal positions as given in Eq. (9).

$$
M^{e}=\left(\begin{array}{c|ccccc} 
& e_{1 R} & e_{2 R} & e_{3 R} & e_{4 R} & \tilde{L}_{4 R} \\
\bar{L}_{1 L} & 0 & 0 & 0 & 0 & 0 \\
\bar{L}_{2 L} & 0 & 0 & 0 & m_{24} & 0 \\
\bar{L}_{3 L} & 0 & 0 & 0 & m_{34} & m_{35} \\
\bar{L}_{4 L} & 0 & 0 & m_{43} & 0 & M_{45}^{L} \\
\overline{\tilde{e}}_{4 L} & 0 & m_{52} & m_{53} & M_{54}^{e} & 0
\end{array}\right)=\left(\begin{array}{cccccc} 
& e_{1 R} & e_{2 R} & e_{3 R} & \tilde{L}_{4 R} & e_{4 R} \\
\bar{L}_{1 L} & 0 & 0 & 0 & 0 & 0 \\
\bar{L}_{2 L} & 0 & 0 & 0 & 0 & m_{24} \\
\bar{L}_{3 L} & 0 & 0 & 0 & m_{35} & m_{34} \\
\bar{L}_{4 L} & 0 & 0 & m_{43} & M_{45}^{L} & 0 \\
\overline{\tilde{e}}_{4 L} & 0 & m_{52} & m_{53} & 0 & M_{54}^{e}
\end{array}\right),
$$

We use two methods for diagonalizing the rotated mass matrix of Eq. (9), one of which corresponds to the numerical diagonalization carried out by the singular value decomposition (SVD) and the other is an approximated analytical step-by-step diagonalization. We make use of the numerical SVD diagonalization for the exact diagonalization and perform numerical scans in main body of this work, however it is worth discussing the analytic step-by-step diagonalization as it gives an analytical understanding on how the $\mathrm{SM} \mathrm{Z}$ gauge boson can induce the flavor violating interactions at tree-level with the $S U(2)$ violating mixings, which will be defined in the analytic diagonalization covered in Appendix A. From the comparison between the analytic and numerical computations, we found that the former works quite well and yields results close to the ones obtained from the latter. The comparisons between the analytic and numerical computations for both lepton and quark sectors will be discussed in detail in Appendixes D and E, respectively. The charged lepton sector can be diagonalized by performing the SVD diagonalization as follows:

$$
M^{e \prime}=\operatorname{diag}\left(0, m_{\mu}, m_{\tau}, M_{E_{4}}, M_{\tilde{E}_{4}}\right)=V^{L} M^{e}\left(V^{e}\right)^{\dagger},
$$

where $V^{L}\left(V^{e}\right)$ is the mixing matrix for the left-handed (righthanded) leptonic fields, defined as follows:

$$
\left(\begin{array}{c}
e_{L} \\
\mu_{L} \\
\tau_{L} \\
E_{4 L} \\
\tilde{E}_{4 L}
\end{array}\right)=V^{L}\left(\begin{array}{c}
e_{1 L} \\
e_{2 L} \\
e_{3 L} \\
e_{4 L} \\
\tilde{e}_{4 L}
\end{array}\right), \quad\left(\begin{array}{c}
e_{R} \\
\mu_{R} \\
\tau_{R} \\
\tilde{E}_{4 R} \\
E_{4 R}
\end{array}\right)=V^{e}\left(\begin{array}{c}
e_{1 R} \\
e_{2 R} \\
e_{3 R} \\
\tilde{e}_{4 R} \\
e_{4 R}
\end{array}\right),
$$

and the numerical mixing matrices $V^{L, e}$ can be expressed by an analytic expression consisting of a series of $V_{i j}^{L, e}$ which describes mixing between $i$ th and $j$ th fermion where $i, j=1,2,3,4,5$ and this will be discussed in Appendix A.

\section{B. Diagonalizing the up-type quark mass matrix}

The initial mass matrix for the up-type quark sector in the flavor basis is given by: 


$$
M^{u}=\left(\begin{array}{c|ccccc} 
& u_{1 R} & u_{2 R} & u_{3 R} & u_{4 R} & \tilde{Q}_{4 R} \\
\hline \bar{Q}_{1 L} & 0 & 0 & 0 & 0 & 0 \\
\bar{Q}_{2 L} & 0 & 0 & 0 & y_{24}^{u} v_{u} & 0 \\
\bar{Q}_{3 L} & 0 & 0 & 0 & y_{34}^{u} v_{u} & x_{34}^{Q} v_{\phi} \\
\bar{Q}_{4 L} & 0 & 0 & y_{43}^{u} v_{u} & 0 & M_{44}^{Q} \\
\bar{u}_{4 L} & 0 & x_{42}^{u} v_{\phi} & x_{43}^{u} v_{\phi} & M_{44}^{u} & 0
\end{array}\right)=\left(\begin{array}{cccccc} 
& u_{1 R} & u_{2 R} & u_{3 R} & \tilde{Q}_{4 R} & u_{4 R} \\
\hline \bar{Q}_{1 L} & 0 & 0 & 0 & 0 & 0 \\
\bar{Q}_{2 L} & 0 & 0 & 0 & 0 & m_{24}^{u} \\
\bar{Q}_{3 L} & 0 & 0 & 0 & m_{35}^{u} & m_{34}^{u} \\
\bar{Q}_{4 L} & 0 & 0 & m_{43}^{u} & M_{44}^{Q} & 0 \\
\overline{\tilde{u}}_{4 L} & 0 & m_{52}^{u} & m_{53}^{u} & 0 & M_{44}^{u}
\end{array}\right),
$$

The mass matrix of Eq. (12) in the flavor basis is exactly consistent with the one corresponding to the charged lepton sector excepting for a few substitutions $y^{e} \rightarrow y^{u}, v_{d} \rightarrow v_{u}$, $x^{L} \rightarrow x^{Q}$ and $x^{e} \rightarrow x^{u}$. The analytic mixing matrix for the up-quark sector is exactly same as the one for the charged lepton sector, however unlike the charged lepton sector, diagonalizing the mass matrix for the up-quark sector requires more caution as some numerical off-diagonal elements of order unity. This feature is resulted from the some off-diagonal elements arising as a result of mixing between the heavy top quark mass and the other heavy exotic up-type quark masses are of order unitary, not small enough to obtain precise results in the perturbative diagonalization when compared to the charged lepton sector. We discuss this feature in Appendix E by comparing a numerical mixing matrix obtained from the SVD with the one resulting from the analytic diagonalization. Then we can numerically diagonalize the up-type mass matrix by using the SVD diagonalization as follows:

$$
\left(\begin{array}{c}
u_{L} \\
c_{L} \\
t_{L} \\
U_{4 L} \\
\tilde{U}_{4 L}
\end{array}\right)=V_{L}^{u}\left(\begin{array}{c}
u_{1 L} \\
u_{2 L} \\
u_{3 L} \\
u_{4 L} \\
\tilde{u}_{4 L}
\end{array}\right), \quad\left(\begin{array}{c}
u_{R} \\
c_{R} \\
t_{R} \\
\tilde{U}_{4 R} \\
U_{4 R}
\end{array}\right)=V_{R}^{u}\left(\begin{array}{c}
u_{1 R} \\
u_{2 R} \\
u_{3 R} \\
\tilde{u}_{4 R} \\
u_{4 R}
\end{array}\right)
$$

where the symbol $L$ means left-handed doublet and $e$ denotes right-handed singlet in the charged lepton sector, however it is worth mentioning that the above described notation used in the lepton sector becomes complicated in the quark sector since the mass matrices for the up- and down-type quark sectors have a different form, so we change the mixing notation by $V_{L(R)}^{u, d}$ instead of $V^{Q}$. The analytic diagonalizations for the up-quark sector will be discussed in Appendix B.

\section{Diagonalizing the down-type quark mass matrix}

A nice feature of the model under consideration is that it can predict the CKM mixing matrix and this feature is mainly based on the mixings derived from the downtype quark mass matrix as we will see soon. A quite encouraging feature is that all the mixings among the three SM generations in the down-type quark sector can be accessible even though the first generation of the downtype quark sector remains massless and this feature is quite naturally attributed to this model with the vectorlike family. We start from two mass matrices, one of which is for the up-quark sector whereas the another one is for the down-quark sector.

$$
M^{u}=\left(\begin{array}{c|ccccc} 
& u_{1 R} & u_{2 R} & u_{3 R} & u_{4 R} & \tilde{Q}_{4 R} \\
\hline \bar{Q}_{1 L} & 0 & 0 & 0 & 0 & 0 \\
\bar{Q}_{2 L} & 0 & 0 & 0 & y_{24}^{u} v_{u} & 0 \\
\bar{Q}_{3 L} & 0 & 0 & 0 & y_{34}^{u} v_{u} & x_{34}^{Q} v_{\phi} \\
\bar{Q}_{4 L} & 0 & 0 & y_{43}^{u} v_{u} & 0 & M_{44}^{Q} \\
\overline{\tilde{u}}_{4 L} & 0 & x_{42}^{u} v_{\phi} & x_{43}^{u} v_{\phi} & M_{44}^{u} & 0
\end{array}\right), \quad M^{d}=\left(\begin{array}{cc|cccc} 
& d_{1 R} & d_{2 R} & d_{3 R} & d_{4 R} & \tilde{Q}_{4 R} \\
\bar{Q}_{1 L} & 0 & 0 & 0 & y_{14}^{d} v_{d} & 0 \\
\bar{Q}_{2 L} & 0 & 0 & 0 & y_{24}^{d} v_{d} & 0 \\
\bar{Q}_{3 L} & 0 & 0 & 0 & y_{34}^{d} v_{d} & x_{34}^{Q} v_{\phi} \\
\bar{Q}_{4 L} & 0 & 0 & y_{43}^{d} v_{d} & 0 & M_{44}^{Q} \\
\overline{\tilde{d}}_{4 L} & 0 & x_{42}^{d} v_{\phi} & x_{43}^{d} v_{\phi} & M_{44}^{d} & 0
\end{array}\right),
$$

This difference between the mass matrices for the up-type and down-type quark sectors was noticed for the first time in [6]. The first property we need to focus on is the fifth column of both mass matrices is exactly same. The zeros appearing in the fifth column of both are the common elements shared by both up- and down-type quark mass matrices since the quark doublets as well as the fourth vectorlike quark doublets contribute equally to both sectors. For the up-type quark sector, we were able to rotate further between $Q_{1 L}$ and $Q_{2 L}$ to vanish $y_{14}^{u} v_{u}$, however this 
rotation simply remixes $y_{14}^{d} v_{d}$ and $y_{24}^{d} v_{d}$, so both the downtype Yukawa terms survive. For the lower-left $2 \times 3$ block of the down-type quark mass matrix, the same zeros can appear since the down-type quarks $d_{1,2,3 R}$ have a different mixing angle against that for the up-type quarks $u_{1,2,3 R}$. The down-type mass matrix $M^{d}$ of Eq. (14) can be diagonalized by the numerical SVD diagonalization as follows:

$$
\left(\begin{array}{c}
d_{L} \\
s_{L} \\
b_{L} \\
D_{4 L} \\
\tilde{D}_{4 L}
\end{array}\right)=V_{L}^{d}\left(\begin{array}{c}
d_{1 L} \\
d_{2 L} \\
d_{3 L} \\
d_{4 L} \\
\tilde{d}_{4 L}
\end{array}\right), \quad\left(\begin{array}{c}
d_{R} \\
s_{R} \\
b_{R} \\
D_{4 R} \\
\tilde{D}_{4 R}
\end{array}\right)=V_{R}^{d}\left(\begin{array}{c}
d_{1 R} \\
d_{2 R} \\
d_{3 R} \\
\tilde{d}_{4 R} \\
d_{4 R}
\end{array}\right) .
$$

The analytic diagonalization for the down-quark sector is discussed in Appendix C. We will see that a numerical mixing matrix derived by the SVD is quite close to one by the analytic diagonalization in Appendix E. In Appendix E we confirm that even though the numerical mixing matrix $V_{L}^{d}$ can have all mixings among the three SM generations, the $Z$ coupling constants $D_{L}^{d \prime}$ in the mass basis will have zeros in the first column and row due to some internal cancellations. Therefore the whole structure of $D_{L}^{d \prime}$ is exactly same as the other $Z$ coupling constants $D_{L, R}^{u \prime}$ and $D_{R}^{d \prime}$ in the mass basis, so we verify that the SM $Z$ physics does not get affected by any specific basis we choose. This feature will be discussed again in the next section as well as in Appendix E.

\section{THE SM $Z$ GAUGE BOSON INTERACTIONS WITH THE VECTORLIKE FAMILY}

One of our main motivations of this work is to study flavor violating processes mediated by the $Z$ gauge boson in order to constrain the mass range of the vectorlike fermions. It is worth reminding that the neutral $Z^{\prime}$ gauge boson does not appear in the particle spectrum of this model due to the global $U(1)^{\prime}$ symmetry of the theory under consideration. It is worth mentioning that the treelevel flavor violating $Z$ decays are absent in the SM, independently of the fermion mixings, as can be seen from Eq. (16) shown below:

$$
\mathcal{L}_{\mathrm{SM}}^{Z}=g Z_{\mu} J_{Z}^{\mu}=\frac{g}{c_{w}} Z_{\mu} \sum_{f=e, \mu, \tau} \bar{f} \gamma^{\mu}\left(T^{3}-\sin ^{2} \theta_{w} Q\right) f
$$

Factoring out the prefactor $g / c_{w}$, we can find matrices $D_{L, R}^{e}$, which determine the magnitude of the coupling constant for the $Z$ interactions to either the left-handed or right-handed SM fermions.

$$
\begin{aligned}
& D_{L}^{e}=\left(\begin{array}{c|ccc} 
& e_{1 L} & e_{2 L} & e_{3 L} \\
\hline \bar{e}_{1 L} & \left(-\frac{1}{2}+\sin ^{2} \theta_{w}\right) & 0 & 0 \\
\bar{e}_{2 L} & 0 & \left(-\frac{1}{2}+\sin ^{2} \theta_{w}\right) & 0 \\
\bar{e}_{3 L} & 0 & 0 & \left(-\frac{1}{2}+\sin ^{2} \theta_{w}\right)
\end{array}\right) \\
& D_{R}^{e}=\left(\begin{array}{c|ccc} 
& e_{1 R} & e_{2 R} & e_{3 R} \\
\hline \bar{e}_{1 R} & \left(\sin ^{2} \theta_{w}\right) & 0 & 0 \\
\bar{e}_{2 R} & 0 & \left(\sin ^{2} \theta_{w}\right) & 0 \\
\bar{e}_{3 R} & 0 & 0 & \left(\sin ^{2} \theta_{w}\right)
\end{array}\right)
\end{aligned}
$$

However, this SM $Z$ gauge boson can cause the renormalizable flavor violating interactions with the SM fermions by extending the SM fermion sector by the vectorlike fermions as well as by considering the $S U(2)$ violating mixings defined in Appendix A together. These features will be discussed in detail in the following subsections.

\section{A. FCNC mediated by the SM $Z$ gauge boson in the charged lepton sector with the fourth vectorlike charged leptons}

We can construct an extended the SM $Z$ coupling constants in the charged lepton sector with the vectorlike charged leptons in the flavor basis. 


$$
\begin{aligned}
& D_{L}^{e}=\left(\begin{array}{c|ccccc} 
& e_{1 L} & e_{2 L} & e_{3 L} & e_{4 L} & \tilde{e}_{4 L} \\
\hline \bar{e}_{1 L} & \left(-\frac{1}{2}+\sin ^{2} \theta_{w}\right) & 0 & 0 & 0 & 0 \\
\bar{e}_{2 L} & 0 & \left(-\frac{1}{2}+\sin ^{2} \theta_{w}\right) & 0 & 0 & 0 \\
\bar{e}_{3 L} & 0 & 0 & \left(-\frac{1}{2}+\sin ^{2} \theta_{w}\right) & 0 & 0 \\
\bar{e}_{4 L} & 0 & 0 & 0 & \left(-\frac{1}{2}+\sin ^{2} \theta_{w}\right) & 0 \\
\bar{e}_{4 L} & 0 & 0 & 0 & 0 & \left(\sin ^{2} \theta_{w}\right)
\end{array}\right) \\
& D_{R}^{e}=\left(\begin{array}{c|ccccc} 
& e_{1 R} & e_{2 R} & e_{3 R} & \tilde{e}_{4 R} & e_{4 R} \\
\hline \bar{e}_{1 R} & \left(\sin ^{2} \theta_{w}\right) & 0 & 0 & 0 & 0 \\
\bar{e}_{2 R} & 0 & \left(\sin ^{2} \theta_{w}\right) & 0 & 0 & 0 \\
\bar{e}_{3 R} & 0 & 0 & \left(\sin ^{2} \theta_{w}\right) & 0 & 0 \\
\overline{\tilde{e}}_{4 R} & 0 & 0 & 0 & \left(-\frac{1}{2}+\sin ^{2} \theta_{w}\right) & 0 \\
\bar{e}_{4 R} & 0 & 0 & 0 & 0 & \left(\sin ^{2} \theta_{w}\right)
\end{array}\right)
\end{aligned}
$$

where it is worth reminding the reader that the order of the left-handed fermions is 12345 , whereas that of the right-handed fermions is 12354 (This ordering is stressed in Appendix A). An important feature of this SM $Z$ coupling constants of Eq. (18) is the couplings constants are naturally determined, based on the nature of the vectorlike charged leptons, without imposing neither any other symmetry nor other constraints. Therefore, the SM $Z$ coupling constants of Eq. (18) are not the identity matrix anymore unlike the case for the SM charged leptons. From these considerations, it follows that there can exist nonzero off-diagonal elements in the mass basis by operating the $S U(2)$ violating mixings. Reminding the mixing matrices for the left- or right-handed charged leptons of Eq. (20), the coupling constant in the mass basis $\left(D_{L, R}^{e \prime}\right)$ can be written as follows:

$$
\begin{aligned}
D_{L}^{e \prime} & =V^{L} D_{L}^{e}\left(V^{L}\right)^{\dagger} \\
& =V_{45}^{L} V_{23}^{L} V_{25}^{L} V_{35}^{L} V_{34}^{L} D_{L}^{e}\left(V_{34}^{L}\right)^{\dagger}\left(V_{35}^{L}\right)^{\dagger}\left(V_{25}^{L}\right)^{\dagger}\left(V_{23}^{L}\right)^{\dagger}\left(V_{45}^{L}\right)^{\dagger} \\
D_{R}^{e \prime} & =V^{e} D_{R}^{e}\left(V^{e}\right)^{\dagger} \\
& =V_{54}^{e} V_{23}^{e} V_{25}^{e} V_{35}^{e} V_{24}^{e} V_{34}^{e} D_{R}^{e}\left(V_{34}^{e}\right)^{\dagger}\left(V_{24}^{e}\right)^{\dagger}\left(V_{35}^{e}\right)^{\dagger}\left(V_{25}^{e}\right)^{\dagger}\left(V_{23}^{e}\right)^{\dagger}\left(V_{54}^{e}\right)^{\dagger}
\end{aligned}
$$

It is possible to make the SM $Z$ coupling constants of Eq. (19) simpler by using the $S U(2)$ conserving mixing as it just remixes an identity matrix.

$$
\begin{aligned}
& D_{L}^{e \prime}=V_{45}^{L} V_{23}^{L} V_{25}^{L} V_{35}^{L} D_{L}^{e}\left(V_{35}^{L}\right)^{\dagger}\left(V_{25}^{L}\right)^{\dagger}\left(V_{23}^{L}\right)^{\dagger}\left(V_{45}^{L}\right)^{\dagger} \\
& D_{R}^{e \prime}=V_{54}^{e} V_{23}^{e} V_{25}^{e} V_{35}^{e} D_{R}^{e}\left(V_{35}^{e}\right)^{\dagger}\left(V_{25}^{e}\right)^{\dagger}\left(V_{23}^{e}\right)^{\dagger}\left(V_{54}^{e}\right)^{\dagger}
\end{aligned}
$$

However, the following mixing matrices after the $S U(2)$ violating mixings $V_{35}^{L, e}$ must be conserved since all of them contribute to the off-diagonal elements of the coupling constants in the mass basis. It is insightful to look at the coupling constants $D_{L, R}^{e \prime}$ in the mass basis (We substitute $\left(-1 / 2+\sin ^{2} \theta_{w}\right)$ by $a$ and $\left(\sin ^{2} \theta_{w}\right)$ by $b$ and assume the mixing angles $\theta_{35,25,23,45(54)}^{L, e}$ are small enough to approximate for simplicity). 


$$
\begin{aligned}
D_{L}^{e \prime} \approx & \left(\begin{array}{ccccc}
a & 0 & 0 & 0 & 0 \\
0 & a\left(1+\theta_{23}^{L 2}\right)+b \theta_{25}^{L 2} & b \theta_{25}^{L} \theta_{35}^{L} & b \theta_{25}^{L} \theta_{45}^{L} & (a-b) \theta_{25}^{L} \\
0 & b \theta_{25}^{L} \theta_{35}^{L} & a\left(1+\theta_{23}^{L 2}\right)+b \theta_{35}^{L 2} & b \theta_{35}^{L} \theta_{45}^{L} & (a-b) \theta_{35}^{L} \\
0 & b \theta_{25}^{L} \theta_{45}^{L} & b \theta_{35}^{L} \theta_{45}^{L} & a+b \theta_{45}^{L 2} & (a-b) \theta_{45}^{L} \\
0 & (a-b) \theta_{25}^{L} & (a-b) \theta_{35}^{L} & (a-b) \theta_{45}^{L} & b+a\left(\theta_{25}^{L 2}+\theta_{35}^{L 2}+\theta_{45}^{L 2}\right)
\end{array}\right) \\
D_{R}^{e \prime} \approx & \left(\begin{array}{ccccc}
b & 0 & 0 & 0 & 0 \\
0 & b\left(1+\theta_{23}^{e 2}\right)+a \theta_{25}^{e 2} & a \theta_{25}^{e} \theta_{35}^{e} & (b-a) \theta_{25}^{e} & -a \theta_{25}^{e} \theta_{54}^{e} \\
0 & a \theta_{25}^{e} \theta_{35}^{e} & b\left(1+\theta_{23}^{e 2}\right)+a \theta_{35}^{e 2} & (b-a) \theta_{35}^{e} & -a \theta_{35}^{e} \theta_{54}^{e} \\
0 & (b-a) \theta_{25}^{e} & (b-a) \theta_{35}^{e} & a+b\left(\theta_{25}^{e 2}+\theta_{35}^{e 2}+\theta_{54}^{e 2}\right) & (a-b) \theta_{54}^{e} \\
0 & -a \theta_{25}^{e} \theta_{54}^{e} & -a \theta_{35}^{e} \theta_{54}^{e} & (a-b) \theta_{54}^{e} & b+a \theta_{54}^{e 2}
\end{array}\right)
\end{aligned}
$$

There are two important features we can read off from the SM $Z$ coupling constants of Eq. (21); the first is the diagonal elements $(a, a, a, a, b)$ and $(b, b, b, a, b)$ get hardly affected by the small mixing angles and the second is magnitude of the off-diagonal elements are very weak as the mixing angles are defined by the ratio between the Yukawa and the vectorlike masses. Therefore, we can imply the flavor violating mixing mediated by the SM $Z$ gauge boson in the mass basis. Using the SM $Z$ coupling constants in the mass basis, we can draw the Feynman diagram for $\tau \rightarrow \mu \mu \mu$ and $Z \rightarrow \mu \tau$ decay at treelevel and this will be discussed in the next section.

\section{B. FCNC mediated by the SM $Z$ gauge boson in the quark sector with the fourth vectorlike quarks}

The quark sector have two different mass matrices for the up- and down-type quark sector. We start from the up-type quark sector first. The SM $Z$ coupling constants for the up-type quark sector are given by:

$$
\begin{aligned}
& \left(\begin{array}{c|ccccc} 
& u_{1 L} & u_{2 L} & u_{3 L} & u_{4 L} & \tilde{u}_{4 L} \\
\hline \bar{u}_{1 L} & \left(\frac{1}{2}-\frac{2}{3} \sin ^{2} \theta_{w}\right) & 0 & 0 & 0 & 0 \\
\bar{u}_{2 L} & 0 & \left(\frac{1}{2}-\frac{2}{3} \sin ^{2} \theta_{w}\right) & 0 & 0 & 0 \\
\bar{u}_{3 L} & 0 & 0 & \left(\frac{1}{2}-\frac{2}{3} \sin ^{2} \theta_{w}\right) & 0 & 0 \\
\bar{u}_{4 L} & 0 & 0 & 0 & \left(\frac{1}{2}-\frac{2}{3} \sin ^{2} \theta_{w}\right) & 0 \\
\bar{u}_{4 L} & 0 & 0 & 0 & 0 & \left(-\frac{2}{3} \sin ^{2} \theta_{w}\right)
\end{array}\right) \\
D_{R}^{u} & =\left(\begin{array}{c|ccccc}
u_{1 R} & u_{2 R} & u_{3 R} & \tilde{u}_{4 R} & u_{4 R} \\
\hline \bar{u}_{1 R} & \left(-\frac{2}{3} \sin ^{2} \theta_{w}\right) & 0 & 0 & 0 & 0 \\
\bar{u}_{2 R} & 0 & \left(-\frac{2}{3} \sin ^{2} \theta_{w}\right) & 0 & 0 & 0 \\
\bar{u}_{3 R} & 0 & 0 & \left(-\frac{2}{3} \sin ^{2} \theta_{w}\right) & 0 & 0 \\
\overline{\tilde{u}}_{4 R} & 0 & 0 & 0 & \left(\frac{1}{2}-\frac{2}{3} \sin ^{2} \theta_{w}\right) & 0 \\
\bar{u}_{4 R} & 0 & 0 & 0 & 0 & \left(-\frac{2}{3} \sin ^{2} \theta_{w}\right)
\end{array}\right)
\end{aligned}
$$

The up-type quark mass matrix is exactly same as the one for the charged lepton, so we can simply follow the mixing matrices given in Eq. (B2). Then the SM $Z$ gauge coupling constants in the mass basis are defined by:

$$
\begin{aligned}
D_{L}^{u \prime} & =V_{L}^{u} D_{L}^{u} V_{L}^{u \dagger} \\
& =\left(V_{L}^{u}\right)_{45}\left(V_{L}^{u}\right)_{23}\left(V_{L}^{u}\right)_{25}\left(V_{L}^{u}\right)_{35}\left(V_{L}^{u}\right)_{34} D_{L}^{u}\left(V_{L}^{u}\right)_{34}^{\dagger}\left(V_{L}^{u}\right)_{35}^{\dagger}\left(V_{L}^{u}\right)_{25}^{\dagger}\left(V_{L}^{u}\right)_{23}^{\dagger}\left(V_{L}^{u}\right)_{45}^{\dagger} \\
& =\left(V_{L}^{u}\right)_{45}\left(V_{L}^{u}\right)_{23}\left(V_{L}^{u}\right)_{25}\left(V_{L}^{u}\right)_{35} D_{L}^{u}\left(V_{L}^{u}\right)_{35}^{\dagger}\left(V_{L}^{u}\right)_{25}^{\dagger}\left(V_{L}^{u}\right)_{23}^{\dagger}\left(V_{L}^{u}\right)_{45}^{\dagger} \\
D_{R}^{u \prime} & =V_{R}^{u} D_{R}^{u} V_{R}^{u \dagger} \\
& =\left(V_{R}^{u}\right)_{54}\left(V_{R}^{u}\right)_{23}\left(V_{R}^{u}\right)_{25}\left(V_{R}^{u}\right)_{35}\left(V_{R}^{u}\right)_{24}\left(V_{R}^{u}\right)_{34} D_{R}^{u}\left(V_{R}^{u}\right)_{34}^{\dagger}\left(V_{R}^{u}\right)_{24}^{\dagger}\left(V_{R}^{u}\right)_{35}^{\dagger}\left(V_{R}^{u}\right)_{25}^{\dagger}\left(V_{R}^{u}\right)_{23}^{\dagger}\left(V_{R}^{u}\right)_{54}^{\dagger} \\
& =\left(V_{R}^{u}\right)_{54}\left(V_{R}^{u}\right)_{23}\left(V_{R}^{u}\right)_{25}\left(V_{R}^{u}\right)_{35} D_{R}^{u}\left(V_{R}^{u}\right)_{35}^{\dagger}\left(V_{R}^{u}\right)_{25}^{\dagger}\left(V_{R}^{u}\right)_{23}^{\dagger}\left(V_{R}^{u}\right)_{54}^{\dagger}
\end{aligned}
$$


The SM $Z$ gauge coupling constants for the up-quark sector in the mass basis can be seen by (We substitute $(1 / 2-$ $\left.2 / 3 \sin ^{2} \theta_{w}\right)$ by $c$ and $\left(-2 / 3 \sin ^{2} \theta_{w}\right)$ by $d$ and assume the mixing angles $\theta_{35,25,23,45(54) L, R}^{u}$ are small enough to approximate for simplicity):

$$
\begin{aligned}
D_{L}^{u \prime} \approx & \left(\begin{array}{ccccc}
c & 0 & 0 & 0 & 0 \\
0 & c & d \theta_{25 L}^{u} \theta_{35 L}^{u} & d \theta_{25 L}^{u} \theta_{45 L}^{u} & (c-d) \theta_{25 L}^{u} \\
0 & d \theta_{25 L}^{u} \theta_{35 L}^{u} & c & d \theta_{35 L}^{u} \theta_{45 L}^{u} & (c-d) \theta_{35 L}^{u} \\
0 & d \theta_{25 L}^{u} \theta_{45 L}^{u} & d \theta_{35 L}^{u} \theta_{45 L}^{u} & c & (c-d) \theta_{45 L}^{u} \\
0 & (c-d) \theta_{25 L}^{u} & (c-d) \theta_{35 L}^{u} & (c-d) \theta_{45 L}^{u} & d
\end{array}\right) \\
D_{R}^{u \prime} \approx & \left(\begin{array}{ccccc}
d & 0 & 0 & 0 & 0 \\
0 & d & c \theta_{25 R}^{u} \theta_{35 R}^{u} & (d-c) \theta_{25 R}^{u} & -c \theta_{25 R}^{u} \theta_{54 R}^{u} \\
0 & c \theta_{25 R}^{u} \theta_{35 R}^{u} & d & (d-c) \theta_{35 R}^{u} & -c \theta_{35 R}^{u} \theta_{54 R}^{u} \\
0 & (d-c) \theta_{25 R}^{u} & (d-c) \theta_{35 R}^{u} & c & (c-d) \theta_{54 R}^{u} \\
0 & -c \theta_{25 R}^{u} \theta_{54 R}^{u} & -c \theta_{35 R}^{u} \theta_{54 R}^{u} & (c-d) \theta_{54 R}^{u} & d
\end{array}\right)
\end{aligned}
$$

Next, we focus on the down-type quark mass matrix and the left-handed mixing matrices for that is different when compared to other left-handed mixing matrices for the up-type or charged lepton mass matrix in that it can reach to all mixings among the three SM generations. Keeping that in mind, we start from the SM $Z$ coupling constants for the down-type quarks.

$$
\begin{aligned}
& D_{L}^{d}=\left(\begin{array}{l|ccccc} 
& d_{1 L} & d_{2 L} & d_{3 L} & d_{4 L} & \tilde{d}_{4 L} \\
\hline \bar{d}_{1 L} & \left(-\frac{1}{2}+\frac{1}{3} \sin ^{2} \theta_{w}\right) & 0 & 0 & 0 & 0 \\
\bar{d}_{2 L} & 0 & \left(-\frac{1}{2}+\frac{1}{3} \sin ^{2} \theta_{w}\right) & 0 & 0 & 0 \\
\bar{d}_{3 L} & 0 & 0 & \left(-\frac{1}{2}+\frac{1}{3} \sin ^{2} \theta_{w}\right) & 0 & 0 \\
\bar{d}_{4 L} & 0 & 0 & 0 & \left(-\frac{1}{2}+\frac{1}{3} \sin ^{2} \theta_{w}\right) & 0 \\
\overline{\tilde{d}}_{4 L} & 0 & 0 & 0 & 0 & \left(\frac{1}{3} \sin ^{2} \theta_{w}\right)
\end{array}\right) \\
& D_{R}^{d}=\left(\begin{array}{c|ccccc} 
& d_{1 R} & d_{2 R} & d_{3 R} & \tilde{d}_{4 R} & d_{4 R} \\
\hline \bar{d}_{1 R} & \left(\frac{1}{3} \sin ^{2} \theta_{w}\right) & 0 & 0 & 0 & 0 \\
\bar{d}_{2 R} & 0 & \left(\frac{1}{3} \sin ^{2} \theta_{w}\right) & 0 & 0 & 0 \\
\bar{d}_{3 R} & 0 & 0 & \left(\frac{1}{3} \sin ^{2} \theta_{w}\right) & 0 & 0 \\
\overline{\tilde{d}}_{4 R} & 0 & 0 & 0 & \left(-\frac{1}{2}+\frac{1}{3} \sin ^{2} \theta_{w}\right) & 0 \\
\bar{d}_{4 R} & 0 & 0 & 0 & 0 & \left(\frac{1}{3} \sin ^{2} \theta_{w}\right)
\end{array}\right)
\end{aligned}
$$

After simplifying the whole left-handed (right-handed) mixing matrices of Eq. (C6), we obtain the following matrices of $Z$ couplings with quarks

$$
\begin{aligned}
& D_{L}^{d \prime}=V_{L}^{d} D_{L}^{d}\left(V_{L}^{d}\right)^{\dagger} \\
& D_{R}^{d \prime}=V_{R}^{d} D_{R}^{d}\left(V_{R}^{d}\right)^{\dagger}
\end{aligned}
$$

The SM $Z$ coupling constants for the right-handed down-type quarks in the mass basis have the same form given in Eq. (21), whereas those for the left-handed down-type quarks involves 12 and 13 mixings more, so it is worthwhile to look at its complete form (We again substitute $\left(-1 / 2+1 / 3 \sin ^{2} \theta_{w}\right)$ by $e$ and $\left(1 / 3 \sin ^{2} \theta_{w}\right)$ by $f$ and assume all relevant mixing angles are small enough to approximate for simplicity) 


$$
\begin{aligned}
& D_{L}^{d \prime} \approx\left(\begin{array}{ccccc}
e & 0 & 0 & 0 & 0 \\
0 & e & e \theta_{12 L}^{d} \theta_{13 L}^{d}+f \theta_{25 L}^{d} \theta_{35 L}^{d} & -e \theta_{23 L}^{d} \theta_{34 L}^{d}+f \theta_{25 L}^{d} \theta_{45 L}^{d} & (e-f) \theta_{25 L}^{d} \\
0 & e \theta_{12 L}^{d} \theta_{13 L}^{d}+f \theta_{25 L}^{d} \theta_{35 L}^{d} & e & f \theta_{35 L}^{d} \theta_{45 L}^{d} & (e-f) \theta_{35 L}^{d} \\
0 & -e \theta_{23 L}^{d} \theta_{34 L}^{d}+f \theta_{25 L}^{d} \theta_{45 L}^{d} & f \theta_{35 L}^{d} \theta_{45 L}^{d} & e & (e-f) \theta_{45 L}^{d} \\
0 & (e-f) \theta_{25 L}^{d} & (e-f) \theta_{35 L}^{d} & (e-f) \theta_{45 L}^{d} & f
\end{array}\right) \\
& D_{R}^{d \prime} \approx\left(\begin{array}{ccccc}
f & 0 & 0 & 0 & 0 \\
0 & f & e \theta_{25 R}^{d} \theta_{35 R}^{d} & (f-e) \theta_{25 R}^{d} & -e \theta_{25 R}^{d} \theta_{54 R}^{d} \\
0 & e \theta_{25 R}^{d} \theta_{35 R}^{d} & f & (f-e) \theta_{35 R}^{d} & -e \theta_{35 R}^{d} \theta_{54 R}^{d} \\
0 & (f-e) \theta_{25 R}^{d} & (f-e) \theta_{35 R}^{d} & e & (e-f) \theta_{54 R}^{d} \\
0 & -e \theta_{25 R}^{d} \theta_{54 R}^{d} & -e \theta_{35 R}^{d} \theta_{54 R}^{d} & (e-f) \theta_{54 R}^{d} & f
\end{array}\right)
\end{aligned}
$$

where it can confirm two relations hold for the zeros appearing in the first row and column of $D_{L}^{d \prime}: \theta_{13 L}^{d} \simeq$ $\theta_{12 L}^{d} \theta_{23 L}^{d}$ and $\theta_{15 L}^{d} \simeq \theta_{12 L}^{d} \theta_{25 L}^{d}$. Following the analytic mixings given in Eq. (C6), the left-handed down type quark sector can access to all mixings among the three SM generations and this feature is also reflected on a numerical mixing matrix $V_{L}^{d}$ of Eq. (E4). What this implies is the $\mathrm{SM} Z$ physics does not get affected by any specific basis we choose and this will be verified again numerically in Appendix E.

\section{PHENOMENOLOGY IN THE CHARGED LEPTON SECTOR OF THE SM}

Now that we have defined all required mixings and coupling constants in both quark and charged lepton sectors, it is time to discuss the relevant phenomenology. As mentioned in the Introduction, one of our main goals is to study the FCNC observables to constrain the possible mass range of the vectorlike fermions. Given that the second and third generations of SM charged leptons acquire masses through their mixings with the fourth vectorlike charged leptons, we will restrict our analysis to the constraints imposed on the flavor violating decays involving the second and third charged lepton generations such as $\tau \rightarrow \mu \gamma, \tau \rightarrow$ $\mu \mu \mu$ and lastly $Z \rightarrow \mu \tau$ decay.

\section{A. Analytic expression for $\boldsymbol{\tau} \rightarrow \boldsymbol{\mu} \gamma$ decay}

The most important FCNC constraint corresponds to the charged lepton flavor violating (CLFV) $\mu \rightarrow e \gamma$ decay, however we cannot make an appropriate prediction for the constraint as the electron does not acquire a mass in the model under consideration. This is due to the fact that the fermion sector of the model includes two heavy fermionic seesaw mediators. As previously mentioned, adding a fifth vectorlike family to the fermion sector of the model will generate a nonvanishing electron. However in order to keep our model as economical as possible and to simplify our analysis corresponding to the FCNC constraints on vectorlike masses, we restrict to the case of a fourth vectorlike family in the fermionic spectrum. Therefore, in view of the aforementioned considerations, we first consider the CLFV decay $\tau \rightarrow \mu \gamma$ in order to determine how the model parameter space gets affected by the experimental constraint arising from this decay. For the $\tau \rightarrow \mu \gamma$ decay, the leading order contribution appears in the one-loop diagrams since there is no possible contribution at tree-level. Then, all possible Feynman diagrams contributing to the $\tau \rightarrow \mu \gamma$ decay are given in Fig. 5,

where $g_{L L(R R)}$ are the LH-LH (RH-RH) coupling constants defined in the mass basis for the charged lepton sector. These one-loop contributions mediated by the $Z^{\prime}$ boson were studied in one of our previous works [7] and their corresponding analytic prediction for the branching ratio of $\tau \rightarrow \mu \gamma$ decay is given in Eq. (28) [7-11] shown below: 

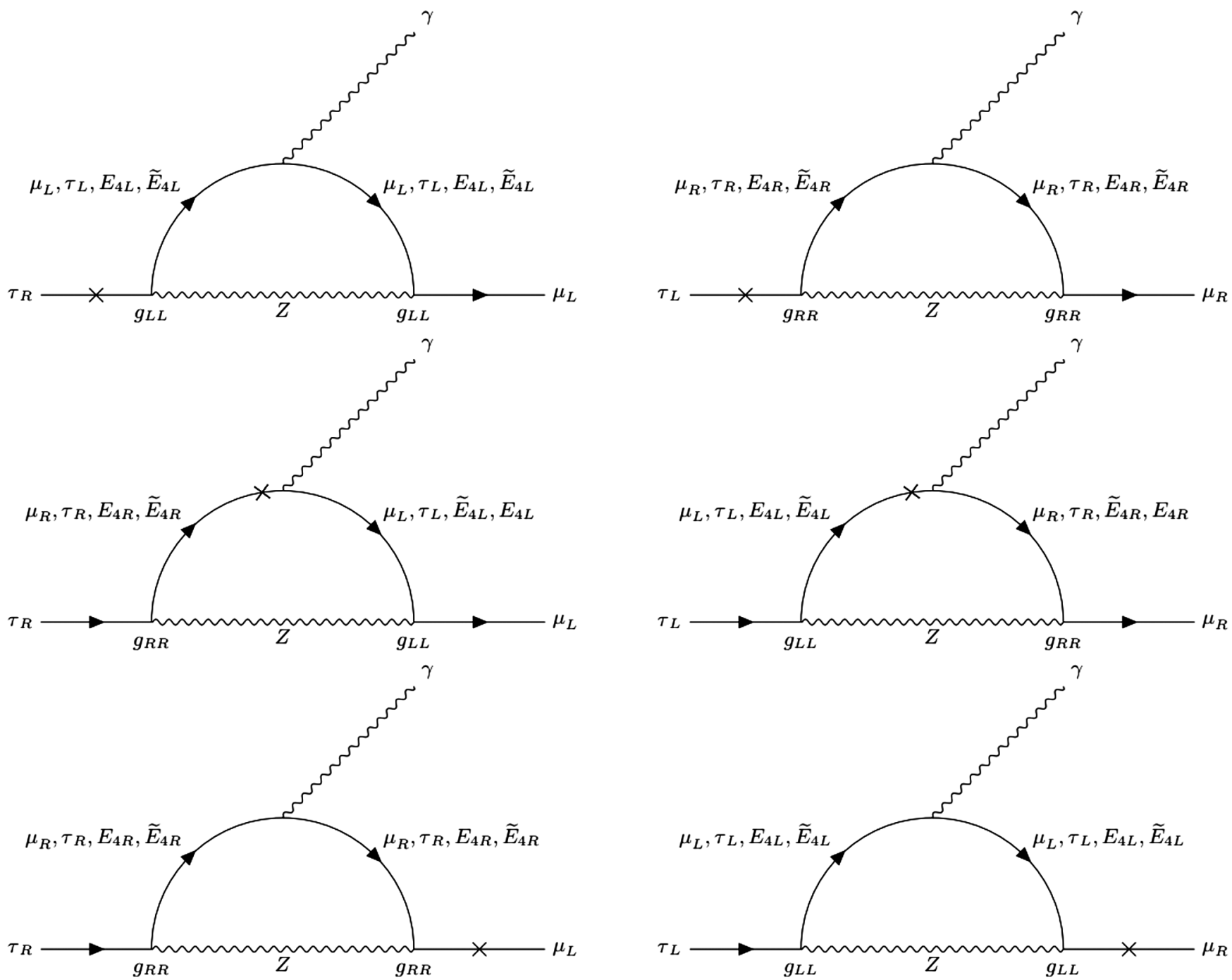

FIG. 5. Diagrams contributing to the charged lepton flavor violation (CLFV) $\tau \rightarrow \mu \gamma$ decay at one-loop level in the mass basis. The cross notation in each diagram means the helicity flip process.

$$
\begin{aligned}
\operatorname{BR}(\tau \rightarrow \mu \gamma)= & \frac{\alpha_{e m}}{1024 \pi^{4}} \frac{m_{\tau}^{5}}{M_{Z}^{4} \Gamma_{\tau}}\left(\mid g_{\tau \mu}^{L} g_{\mu \mu}^{L} F\left(x_{\mu}\right)+g_{\tau \tau}^{L} g_{\tau \mu}^{L} F\left(x_{\tau}\right)+g_{\tau E_{4}}^{L} g_{E_{4} \mu}^{L} F\left(x_{E_{4}}\right)+g_{\tau \tilde{E}_{4}}^{L} g_{\tilde{E}_{4} \mu}^{L} F\left(x_{\tilde{E}_{4}}\right)\right. \\
& +\frac{m_{\mu}}{m_{\tau}} g_{\tau \mu}^{L} g_{\mu \mu}^{L} F\left(x_{\mu}\right)+\frac{m_{\mu}}{m_{\tau}} g_{\tau \tau}^{L} g_{\tau \mu}^{L} F\left(x_{\tau}\right)+\frac{m_{\mu}}{m_{\tau}} g_{\tau E_{4}}^{L} g_{E_{4} \mu}^{L} F\left(x_{E_{4}}\right)+\frac{m_{\mu}}{m_{\tau}} g_{\tau \tilde{E}_{4}}^{L} g_{\tilde{E}_{4} \mu}^{L} F\left(x_{\tilde{E}_{4}}\right) \\
& +\frac{m_{\mu}}{m_{\tau}} g_{\tau \mu}^{L} g_{\mu \mu}^{R} G\left(x_{\mu}\right)+\frac{m_{\tau}}{m_{\tau}} g_{\tau \tau}^{L} g_{\tau \mu}^{R} G\left(x_{\mu}\right)+\frac{M_{E_{4}}}{m_{\tau}} g_{\tau E_{4}}^{L} g_{\tilde{E}_{4} \mu}^{R} G\left(x_{E_{4}}\right)+\left.\frac{M_{\tilde{E}_{4}}}{m_{\tau}} g_{\tau \tilde{E}_{4}}^{L} g_{E_{4} \mu}^{R} G\left(x_{\tilde{E}_{4}}\right)\right|^{2} \\
& +\mid g_{\tau \mu}^{R} g_{\mu \mu}^{R} F\left(x_{\mu}\right)+g_{\tau \tau}^{R} g_{\tau \mu}^{R} F\left(x_{\tau}\right)+g_{\tau E_{4}}^{R} g_{E_{4} \mu}^{R} F\left(x_{E_{4}}\right)+g_{\tau \tilde{E}_{4}}^{R} g_{\tilde{E}_{4} \mu}^{R} F\left(x_{\tilde{E}_{4}}\right) \\
& +\frac{m_{\mu}}{m_{\tau}} g_{\tau \mu}^{R} g_{\mu \mu}^{R} F\left(x_{\mu}\right)+\frac{m_{\mu}}{m_{\tau}} g_{\tau \tau}^{R} g_{\tau \mu}^{R} F\left(x_{\tau}\right)+\frac{m_{\mu}}{m_{\tau}} g_{\tau E_{4}}^{R} g_{E_{4} \mu}^{R} F\left(x_{E_{4}}\right)+\frac{m_{\mu}}{m_{\tau}} g_{\tau \tilde{E}_{4}}^{R} g_{\tilde{E}_{4} \mu}^{R} F\left(x_{\tilde{E}_{4}}\right) \\
& \left.+\frac{m_{\mu}}{m_{\tau}} g_{\tau \mu}^{R} g_{\mu \mu}^{L} G\left(x_{\mu}\right)+\frac{m_{\tau}}{m_{\tau}} g_{\tau \tau}^{R} g_{\tau \mu}^{L} G\left(x_{\mu}\right)+\frac{M_{\tilde{E}_{4}}}{m_{\tau}} g_{\tau E_{4}}^{R} g_{\tilde{E}_{4} \mu}^{L} G\left(x_{\tilde{E}_{4}}\right)+\left.\frac{M_{E_{4}}}{m_{\tau}} g_{\tau \tilde{E}_{4}}^{R} g_{E_{4} \mu}^{L} G\left(x_{E_{4}}\right)\right|^{2}\right),
\end{aligned}
$$

where $\alpha_{e m}$ is the fine structure constant, $\Gamma_{\tau}$ is the total decay width of the tau lepton $\left(\Gamma_{\tau}=5 \times \Gamma\left(\tau_{L}^{-} \rightarrow \nu_{\tau} e_{L}^{-} \bar{\nu}_{e}\right)=\right.$ $2.0 \times 10^{-12}$ ) and $F$ and $G$ are the loop functions defined by: 

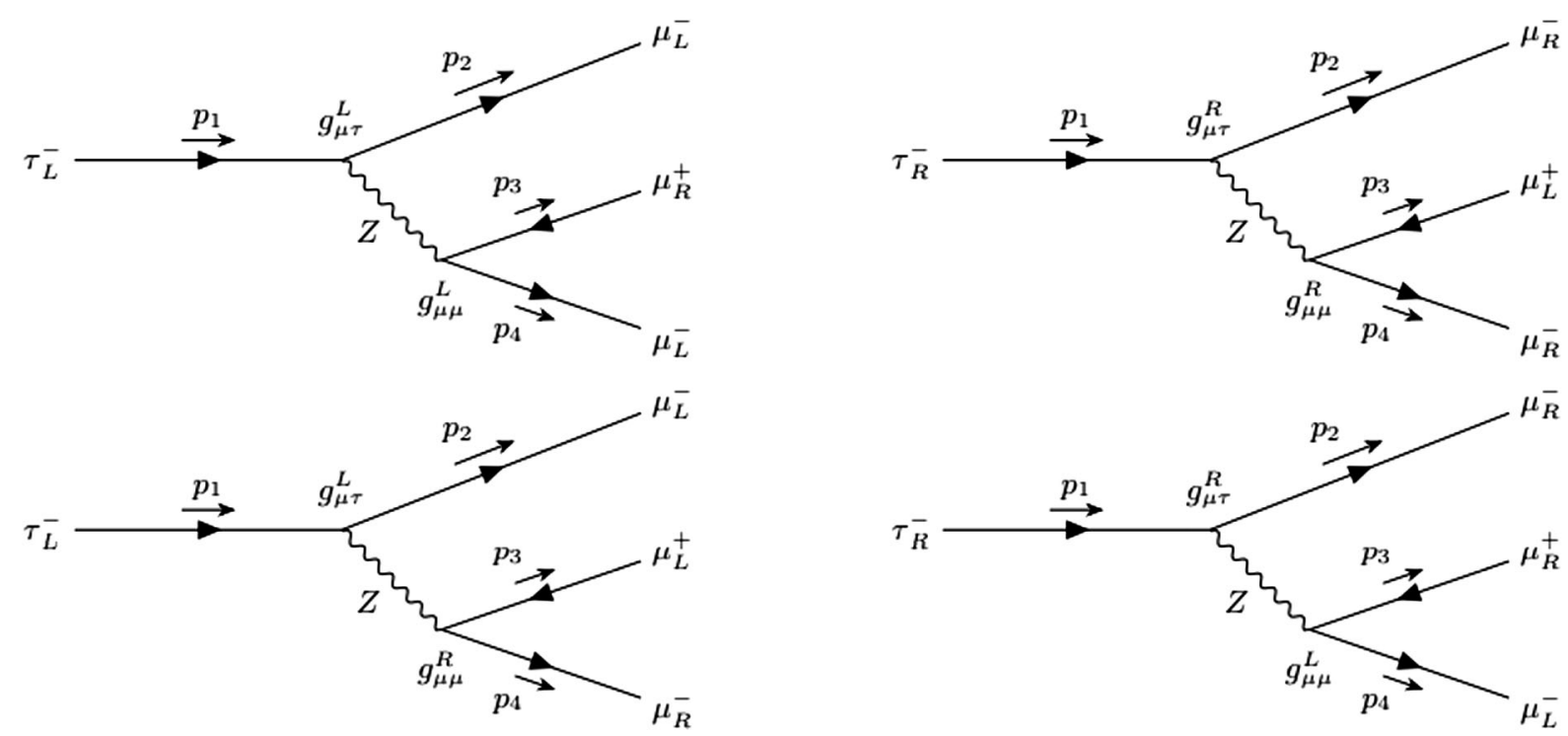

FIG. 6. Diagrams contributing to the charged lepton flavor violation (CLFV) $\tau \rightarrow 3 \mu$ decay at tree-level. We refer to the top-left diagram as $\mathcal{M}_{L L}$ and the top-right as $\mathcal{M}_{R R}$ and similarly for the two below diagrams as $\mathcal{M}_{L R, R L}$, respectively.

$F(x)=\frac{5 x^{4}-14 x^{3}+39 x^{2}-38 x-18 x^{2} \ln x+8}{12(1-x)^{4}}$

$G(x)=\frac{x^{3}+3 x-6 x \ln x-4}{2(1-x)^{3}}, \quad x=\frac{m_{\text {loop }}^{2}}{M_{Z}^{2}}$

where $m_{\text {loop }}$ is the propagating mass of the charged leptons in the loop. The most dominant contributions to the $\tau \rightarrow \mu \gamma$ branching ratio correspond to the terms proportional to $M_{E_{4}\left(\tilde{E}_{4}\right)} / m_{\tau}$ because charged vectorlike leptons are heavier than $200 \mathrm{GeV}$, thus implying that the enhancement factor $M_{E_{4}\left(\tilde{E}_{4}\right)} / m_{\tau}$ makes those contributions much larger than the ones not involving this factor. However, the contributions to the $\tau \rightarrow \mu \gamma$ decay rate involving the terms having the aforementioned proportionality factor do not keep increasing as the vectorlike fermions get heavier since their flavor violating coupling constants get more suppressed at the same time by the small mixing angles, defined by the ratio between Yukawa and vectorlike masses. Therefore, these compensations provide some balanced relation between the vectorlike mass and the coupling of the $Z$ gauge boson with a SM charged antilepton (lepton) and heavy charged vectorlike (antilepton) lepton. The experimental bound for the branching ratio of $\tau \rightarrow \mu \gamma$ decay is given by [12-14]:

$$
\mathrm{BR}(\tau \rightarrow \mu \gamma)_{\mathrm{EXP}}=4.4 \times 10^{-8}
$$

\section{B. Analytic expression for $\tau \rightarrow \mu \mu \mu$ decay}

The other interesting flavor violating decay mode is the $\tau \rightarrow \mu \mu \mu$ decay mediated by the SM $Z$ gauge boson. As the model under consideration has $Z$ mediated renormalizable flavor violating interactions, we can draw the Feynman diagrams for the $\tau \rightarrow \mu \mu \mu$ decay at tree-level as given in Fig. 6.

The contributions shown in Fig. 6 are beyond Standard Model (BSM) effects, thus they need to be computed to set constraints on the model parameter space. In order to derive an analytic expression for the CLFV $\tau \rightarrow 3 \mu$ decay rate mediated by the SM $Z$ gauge boson, we start by writing down its definition as follows:

$$
d \Gamma(\tau \rightarrow 3 \mu)=\frac{1}{2 m_{\tau}} \frac{d^{3} p_{2}}{(2 \pi)^{3} 2 E_{2}} \frac{d^{3} p_{3}}{(2 \pi)^{3} 2 E_{3}} \frac{d^{3} p_{4}}{(2 \pi)^{3} 2 E_{4}}|\mathcal{M}|^{2}(2 \pi)^{4} \delta^{4}\left(p_{1}-p_{2}-p_{3}-p_{4}\right)
$$

Evaluating each polarized diagram in Fig. 6, it yields the following result:

$$
\begin{aligned}
\left|\mathcal{M}_{L L}\right|^{2} & =\left(\frac{g_{\mu \tau}^{L} g_{\mu \mu}^{L}}{4 M_{Z}^{2}}\right)^{2} 256\left(p_{1} \cdot p_{3}\right)\left(p_{2} \cdot p_{4}\right),\left|\mathcal{M}_{R R}\right|^{2}=\left(\frac{g_{\mu \tau}^{R} g_{\mu \mu}^{R}}{4 M_{Z}^{2}}\right)^{2} 256\left(p_{2} \cdot p_{4}\right)\left(p_{1} \cdot p_{3}\right) \\
\left|\mathcal{M}_{L R}\right|^{2} & =\left(\frac{g_{\mu \tau}^{L} g_{\mu \mu}^{R}}{4 M_{Z}^{2}}\right)^{2} 256\left(p_{1} \cdot p_{4}\right)\left(p_{2} \cdot p_{3}\right),\left|\mathcal{M}_{R L}\right|^{2}=\left(\frac{g_{\mu \tau}^{R} g_{\mu \mu}^{L}}{4 M_{Z}^{2}}\right)^{2} 256\left(p_{1} \cdot p_{4}\right)\left(p_{2} \cdot p_{3}\right)
\end{aligned}
$$

We are now ready to determine the squared amplitude averaged and summed over the initial and final spin states. 


$$
\begin{aligned}
\frac{1}{2} \sum_{\text {spin }}|\mathcal{M}|^{2} & =\frac{1}{2}\left(\left|\mathcal{M}_{L L}\right|^{2}+\left|\mathcal{M}_{R R}\right|^{2}+\left|\mathcal{M}_{L R}\right|^{2}+\left|\mathcal{M}_{R L}\right|^{2}\right) \\
& =\frac{8}{M_{Z}^{4}}\left[\left(g_{\mu \tau}^{2 L} g_{\mu \mu}^{2 L}+g_{\mu \tau}^{2 R} g_{\mu \mu}^{2 R}\right)\left(p_{1} \cdot p_{3}\right)\left(p_{2} \cdot p_{4}\right)+\left(g_{\mu \tau}^{2 L} g_{\mu \mu}^{2 R}+g_{\mu \tau}^{2 R} g_{\mu \mu}^{2 L}\right)\left(p_{1} \cdot p_{4}\right)\left(p_{2} \cdot p_{3}\right)\right]
\end{aligned}
$$

The momentum of the particles involved in the $\tau \rightarrow 3 \mu$ are written in the rest frame as follows:

$$
\begin{aligned}
& p_{1}=\left(m_{\tau}, \overrightarrow{0}\right) \\
& p_{2}=\left(E_{2}, \vec{p}_{2}\right) \\
& p_{3}=\left(m_{\tau}-E_{2}-E_{4},-\vec{p}_{2}-\vec{p}_{4}\right) \\
& p_{4}=\left(E_{4}, \vec{p}_{4}\right)
\end{aligned}
$$

Then, we can carry out the inner products of momenta taking into account the momentum conservation $\left(p_{1}=p_{2}+p_{3}+p_{4}\right)$.

$$
\begin{aligned}
& p_{1} \cdot p_{3}=m_{\tau}\left(m_{\tau}-E_{2}-E_{4}\right) \\
& p_{2} \cdot p_{4}=\frac{1}{2}\left(-m_{\tau}^{2}-m_{\mu}^{2}+2 m_{\tau}\left(E_{2}+E_{4}\right)\right) \\
& p_{1} \cdot p_{4}=m_{\tau} E_{4} \\
& p_{2} \cdot p_{3}=\frac{1}{2}\left(m_{\tau}^{2}-m_{\mu}^{2}-2 m_{\tau} E_{4}\right)
\end{aligned}
$$

We can rewrite the squared amplitude in terms of the mass parameters after simplifying the summing over the diverse coupling constants to $g_{1,2}$, respectively $\left(g_{1}=g_{\mu \tau}^{2 L} g_{\mu \mu}^{2 L}+g_{\mu \tau}^{2 R} g_{\mu \mu}^{2 R}, g_{2}=g_{\mu \tau}^{2 L} g_{\mu \mu}^{2 R}+g_{\mu \tau}^{2 R} g_{\mu \mu}^{2 L}\right)$.

$$
\begin{aligned}
\frac{1}{2} \sum_{\text {spin }}\left|\mathcal{M}\left(g_{1}, g_{2}, E_{2}, E_{4}\right)\right|^{2}= & \frac{4}{M_{Z}^{4}}\left[g_{1}\left(m_{\tau}^{2}-m_{\tau}\left(E_{2}+E_{4}\right)\right)\left(-m_{\tau}^{2}-m_{\mu}^{2}+2 m_{\tau}\left(E_{2}+E_{4}\right)\right)\right. \\
& \left.+g_{2}\left(m_{\tau} E_{4}\right)\left(m_{\tau}^{2}-m_{\mu}^{2}-2 m_{\tau} E_{4}\right)\right] .
\end{aligned}
$$

Now it is time to evaluate the three body phase space integral by turning it into an effective two-body phase integral as follows (we simply drop out the prefactor $1 /(2 \pi)^{5}$ for simplicity in this derivation while keeping the prefactor in the computation of the aforementioned partial decay width).

$$
\begin{aligned}
& \frac{d^{3} p_{2}}{2 E_{2}} \frac{d^{3} p_{3}}{2 E_{3}} \frac{d^{3} p_{4}}{2 E_{4}} \delta^{4}\left(p_{1}-p_{2}-p_{3}-p_{4}\right) \\
& =\frac{d^{3} p_{2}}{2 E_{2}} d^{4} p_{3} \Theta\left(p_{3}^{0}\right) \delta\left(p_{3}^{2}\right) \frac{d^{3} p_{4}}{2 E_{4}} \delta^{4}\left(p_{1}-p_{2}-p_{3}-p_{4}\right) \\
& =\frac{d^{3} p_{2}}{2 E_{2}} \frac{d^{3} p_{4}}{2 E_{4}} \Theta\left(p_{1}^{0}-p_{2}^{0}-p_{4}^{0}\right) \delta\left(\left(p_{1}-p_{2}-p_{4}\right)^{2}\right) \\
& =\frac{d^{3} p_{2}}{2 E_{2}} \frac{d^{3} p_{4}}{2 E_{4}} \Theta\left(p_{1}^{0}-p_{2}^{0}-p_{4}^{0}\right) \delta\left(p_{1}^{2}-2 p_{1} \cdot\left(p_{2}+p_{4}\right)+\left(p_{2}^{2}+2 p_{2} \cdot p_{4}+p_{4}^{2}\right)\right) \\
& =\frac{d^{3} p_{2}}{2 E_{2}} \frac{d^{3} p_{4}}{2 E_{4}} \Theta\left(p_{1}^{0}-p_{2}^{0}-p_{4}^{0}\right) \delta\left(m_{\tau}^{2}+2 m_{\mu}^{2}-2 m_{\tau}\left(E_{2}+E_{4}\right)+2\left(E_{2} E_{4}-\left|\vec{p}_{2}\right|\left|\vec{p}_{4}\right| \cos \theta\right)\right) \\
& =\frac{d^{3} p_{2}}{2 E_{2}} \frac{d^{3} p_{4}}{2 E_{4}} \Theta\left(p_{1}^{0}-p_{2}^{0}-p_{4}^{0}\right) \frac{1}{2\left|\vec{p}_{2}\right|\left|\vec{p}_{4}\right|} \delta\left(\frac{m_{\tau}^{2}+2 m_{\mu}^{2}-2 m_{\tau}\left(E_{2}+E_{4}\right)+2 E_{2} E_{4}}{2\left|\vec{p}_{2}\right|\left|\vec{p}_{4}\right|}-\cos \theta\right) .
\end{aligned}
$$

From the delta function, we can determine the integration range by assuming $E_{2} \approx\left|\vec{p}_{2}\right|, E_{4} \approx\left|\vec{p}_{4}\right|$. When cos $\theta=1$, the obtained result is 

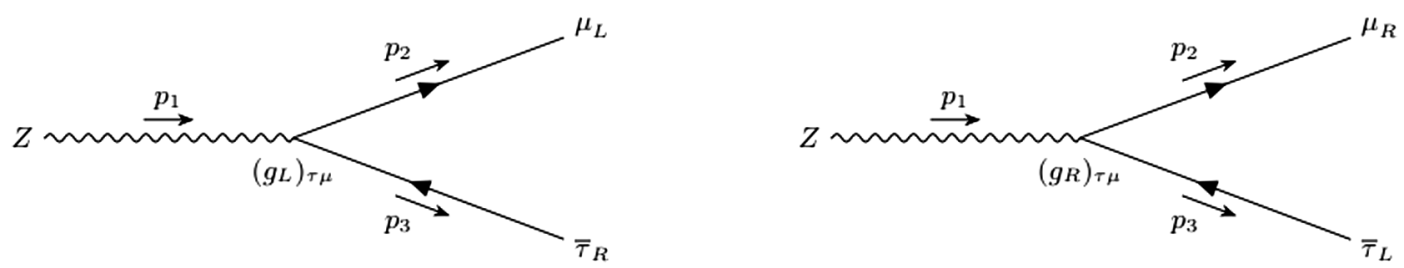

FIG. 7. Diagrams contributing to the charged lepton flavor violation (CLFV) $Z \tau \mu$ decay at tree-level.

$$
m_{\tau}^{2}+2 m_{\mu}^{2}-2 m_{\tau}\left(E_{2}+E_{4}\right)=0 .
$$

From Eq. (38), the integration range can be read off as follows:

$$
\frac{m_{\mu}^{2}}{m_{\tau}} \leq E_{2} \leq \frac{1}{2} m_{\tau}, \quad \frac{1}{2} m_{\tau}+\frac{m_{\mu}^{2}}{m_{\tau}}-E_{2} \leq E_{4} \leq \frac{1}{2} m_{\tau} .
$$

It can be easily understood that once one mass parameter $E_{2}$ is set up by $\frac{1}{2} m_{\tau}$, the energy of the other mass parameters $E_{4}, E_{3}$ must be given by $\frac{m_{\mu}^{2}}{m_{\tau}}, \frac{1}{2} m_{\tau}-\frac{m_{\mu}^{2}}{m_{\tau}}$, respectively. Then, it remains to simplify the effective two body phase space integral as follows.

$$
d^{3} p_{2} d^{3} p_{4}=4 \pi\left|\vec{p}_{2}\right|^{2} d\left|\vec{p}_{2}\right| 2 \pi\left|\vec{p}_{4}\right| d\left|\vec{p}_{4}\right| d \cos \theta, \quad\left|\vec{p}_{2}\right| d\left|\vec{p}_{2}\right|=E_{2} d E_{2}, \quad\left|\vec{p}_{4}\right| d\left|\vec{p}_{4}\right|=E_{4} d E_{4} .
$$

Putting all pieces together, the decay width for the CLFV $\tau \rightarrow 3 \mu$ decay at tree-level after carrying out the $\cos \theta$ integration is given by:

$$
\Gamma(\tau \rightarrow 3 \mu)=\frac{1}{64 m_{\tau} \pi^{3}} \int_{m_{\mu}^{2} / m_{\tau}}^{\frac{1}{2} m_{\tau}} \int_{\frac{1}{2} m_{\tau}+\frac{m_{\mu}^{2}}{m_{\tau}}-E_{2}}^{\frac{1}{2} m_{\tau}}\left(\frac{1}{2} \sum_{\text {spin }}\left|\mathcal{M}\left(g_{1}, g_{2}, E_{2}, E_{4}\right)\right|^{2}\right) d E_{4} d E_{2} .
$$

The experimental bound for the $\tau \rightarrow 3 \mu$ decay is given by [15]:

$$
\mathrm{BR}(\tau \rightarrow 3 \mu)_{\mathrm{EXP}}=2.1 \times 10^{-8} .
$$

\section{Analytic expression for $Z \rightarrow \mu \tau$ decay}

The last FCNC constraint we discuss is the $Z \rightarrow \mu \tau$ decay and diagrams contributing to the $Z \rightarrow \mu \tau$ decay are given in Fig. 7.

As in the CLFV $\tau \rightarrow 3 \mu$ decay mediated by the SM $Z$ gauge boson, this CLFV $Z \rightarrow \mu \tau$ decay is also a new effect and it requires to derive its appropriate prediction from the ground. We can read off the invariant amplitude for each diagram given in Fig. 7. We refer to the left diagram as $\mathcal{M}_{L}$ and the right as $\mathcal{M}_{R}$. Then, the amplitudes are written as follows:

$$
\begin{aligned}
& i \mathcal{M}_{L}=i\left(g_{L}\right)_{\tau \mu} \epsilon_{\mu}\left(p_{1}\right) \bar{u}\left(p_{2}\right) \gamma^{\mu} P_{L} v\left(p_{3}\right), \\
& i \mathcal{M}_{R}=i\left(g_{R}\right)_{\tau \mu} \epsilon_{\mu}\left(p_{1}\right) \bar{u}\left(p_{2}\right) \gamma^{\mu} P_{R} v\left(p_{3}\right) .
\end{aligned}
$$

In order to have the squared amplitude averaged and summed, we square the amplitude given in Eq. (43) as follows:

$$
\begin{aligned}
\frac{1}{3} \sum_{\text {spin }}\left|\mathcal{M}_{L}\right|^{2} & \left.=\frac{1}{3}\left(g_{L}\right)_{\tau \mu}^{2}\left(-g_{\mu \nu}+\frac{p_{1 \mu} p_{1 \nu}}{M_{Z}^{2}}\right) \operatorname{Tr}\left[\left(\not p_{2}+m_{\mu}\right) \gamma^{\mu} P_{L}\left(\not \partial_{3}-m_{\tau}\right) \gamma^{\nu} P_{L}\right)\right] \\
& \simeq \frac{2}{3}\left(g_{L}\right)_{\tau \mu}^{2} M_{Z}^{2} \\
\frac{1}{3} \sum_{\text {spin }}\left|\mathcal{M}_{R}\right|^{2} & \left.=\frac{1}{3}\left(g_{R}\right)_{\tau \mu}^{2}\left(-g_{\mu \nu}+\frac{p_{1 \mu} p_{1 \nu}}{M_{Z}^{2}}\right) \operatorname{Tr}\left[\left(\not p_{2}+m_{\mu}\right) \gamma^{\mu} P_{R}\left(\not p_{3}-m_{\tau}\right) \gamma^{\nu} P_{R}\right)\right] \\
& \simeq \frac{2}{3}\left(g_{R}\right)_{\tau \mu}^{2} M_{Z}^{2} \\
\frac{1}{3} \sum_{\text {spin }}|\mathcal{M}|^{2} & =\frac{1}{3} \sum_{\text {spin }}\left(\left|\mathcal{M}_{L}\right|^{2}+\left|\mathcal{M}_{R}\right|^{2}\right)=\frac{2}{3}\left(\left(g_{L}\right)_{\tau \mu}^{2}+\left(g_{R}\right)_{\tau \mu}^{2}\right) M_{Z}^{2} .
\end{aligned}
$$


Then, the decay rate equation is given by:

$$
\begin{aligned}
d \Gamma(Z \rightarrow \mu \tau) & =\frac{1}{2 M_{Z}} \frac{d^{3} p_{2}}{(2 \pi)^{3} 2 E_{2}} \frac{d^{3} p_{3}}{(2 \pi)^{3} 2 E_{3}}|\mathcal{M}|^{2}(2 \pi)^{4} \delta^{(4)}\left(p_{1}-p_{2}-p_{3}\right) \\
\Gamma(Z \rightarrow \mu \tau) & =\frac{\left|p^{*}\right|}{32 \pi^{2} M_{Z}^{2}} \int|\mathcal{M}|^{2} d \Omega \\
& =\frac{M_{Z}}{24 \pi}\left(\left(g_{L}\right)_{\tau \mu}^{2}+\left(g_{R}\right)_{\tau \mu}^{2}\right)
\end{aligned}
$$

where $p^{*} \simeq M_{Z} / 2$. Then, we are ready to write our prediction for the branching ratio of $Z \mu \tau$ decay at tree-level

$$
\operatorname{BR}(Z \rightarrow \mu \tau)=\frac{\Gamma(Z \rightarrow \mu \tau)}{\Gamma_{Z}} \simeq \frac{1}{2.5} \frac{M_{Z}}{24 \pi}\left(\left(g_{L}\right)_{\tau \mu}^{2}+\left(g_{R}\right)_{\tau \mu}^{2}\right)
$$

where $\Gamma_{Z}$ is the total decay width of the $\mathrm{SM} Z$ gauge boson $\left(\Gamma_{Z} \simeq 2.5 \mathrm{GeV}\right)$. The experimental bound of the CLFV $Z \rightarrow \mu \tau$ decay is known as [16]:

$$
\mathrm{BR}(Z \rightarrow \mu \tau)_{\mathrm{EXP}}=1.2 \times 10^{-5} .
$$

\section{Numerical analysis for each prediction in the charged lepton sector}

We have discussed some relevant CLFV decay modes such as the $\tau \rightarrow \mu \gamma, \tau \rightarrow 3 \mu$ and $Z \rightarrow \mu \tau$ from a theoretical point of view. By defining the renormalizable flavor violating interactions we showed that it is possible for the new physics to arise in a simple scenario thanks to the presence of vectorlike charged leptons, which play a crucial role for these CLFV decay modes to happen. It is an encouraging feature that the mass range of the vectorlike charged leptons can be constrained by the experimental bound of the CLFV decays and numerical scans for this feature will be discussed in detail in the following subsection.

\section{Free parameter setup}

For the numerical scan for the charged lepton sector in Table II, we first proceed to set up a possible mass range of the mass parameters of Eq. (9).

There are a few of features to be noticed before we start the numerical scan.

(1) We assumed a vev for the SM up-type Higgs $H_{u}$ very close to $246 \mathrm{GeV}$, whereas the one of the SM downtype Higgs $H_{d}$ is assumed to be very small compared to the $v_{u}=\left\langle H_{u}\right\rangle$ and is ranged from 1 to $10 \mathrm{GeV}$. The two vevs hold the relation $v_{u}^{2}+v_{d}^{2}=(246 \mathrm{GeV})^{2}$. We made that assumption since we are considering an scenario close to the decoupling limit where the neutral $C P$ even part of $H_{u}$ is mostly identified with the $126 \mathrm{GeV}$ SM like Higgs boson.

(2) As the vev of the singlet flavon $\phi$ is a free parameter, we varied it in the range $[50,200] \mathrm{GeV}$ whereas the mass parameters $m_{35,52,53}$ were varied in a range of values consistent with the observed hierarchical structure of the charged lepton masses. Furthermore, the vectorlike masses are also other free parameters assumed to be larger or equal than $150 \mathrm{GeV}$ in order to successfully fulfill the experimental bounds on exotic charged lepton masses.

(3) What we need to constrain in this numerical scan is the predicted muon and tau masses as well as the 23 mixing angle. For the muon and tau masses, we required that the obtained values of the muon and tau masses to be in the range $[1 \pm 0.1] \times m_{\mu, \tau}$. Considering that the sizeable off-diagonal elements of the PMNS mixing matrix mainly arise from the neutrino sector, all mixing angles in the charged lepton sector are required to be as small as possible and thus we limit them to be lower than 0.2 .

\section{Numerical scan result for the charged lepton sector}

The scanned mass range of the vectorlike charged leptons are shown in Fig. 8.

The first we need to discuss is the experimental bounds for the vectorlike charged leptons appearing in Fig. 8. The darker blue region is the excluded region for the vectorlike charged leptons by $200 \mathrm{GeV}[17,18]$. The vectorlike mass $M_{E_{4}}$ consists of the doublet vectorlike charged leptons $E_{4 L}, \tilde{E}_{4 R}$, whereas the other vectorlike

TABLE II. Initial parameter setup for scanning the mass of the vectorlike charged leptons.

\begin{tabular}{lc}
\hline \hline Mass parameter & Scanned Region $(\mathrm{GeV})$ \\
\hline$y_{24}^{e} v_{d}=m_{24}$ & $\pm[1,10]$ \\
$y_{34}^{e} v_{d}=m_{34}$ & $\pm[1,10]$ \\
$y_{43}^{e} v_{d}=m_{43}$ & $\pm[1,10]$ \\
$x_{34}^{L} v_{\phi}=m_{35}$ & $\pm[50,200]$ \\
$x_{42}^{e} v_{\phi}=m_{52}$ & $\pm[50,200]$ \\
$x_{43}^{e} v_{\phi}=m_{53}$ & $\pm[50,200]$ \\
$M_{45}^{L}$ & $\pm[150,2000]$ \\
$M_{54}^{e}$ & $\pm[150,2000]$ \\
\hline \hline
\end{tabular}



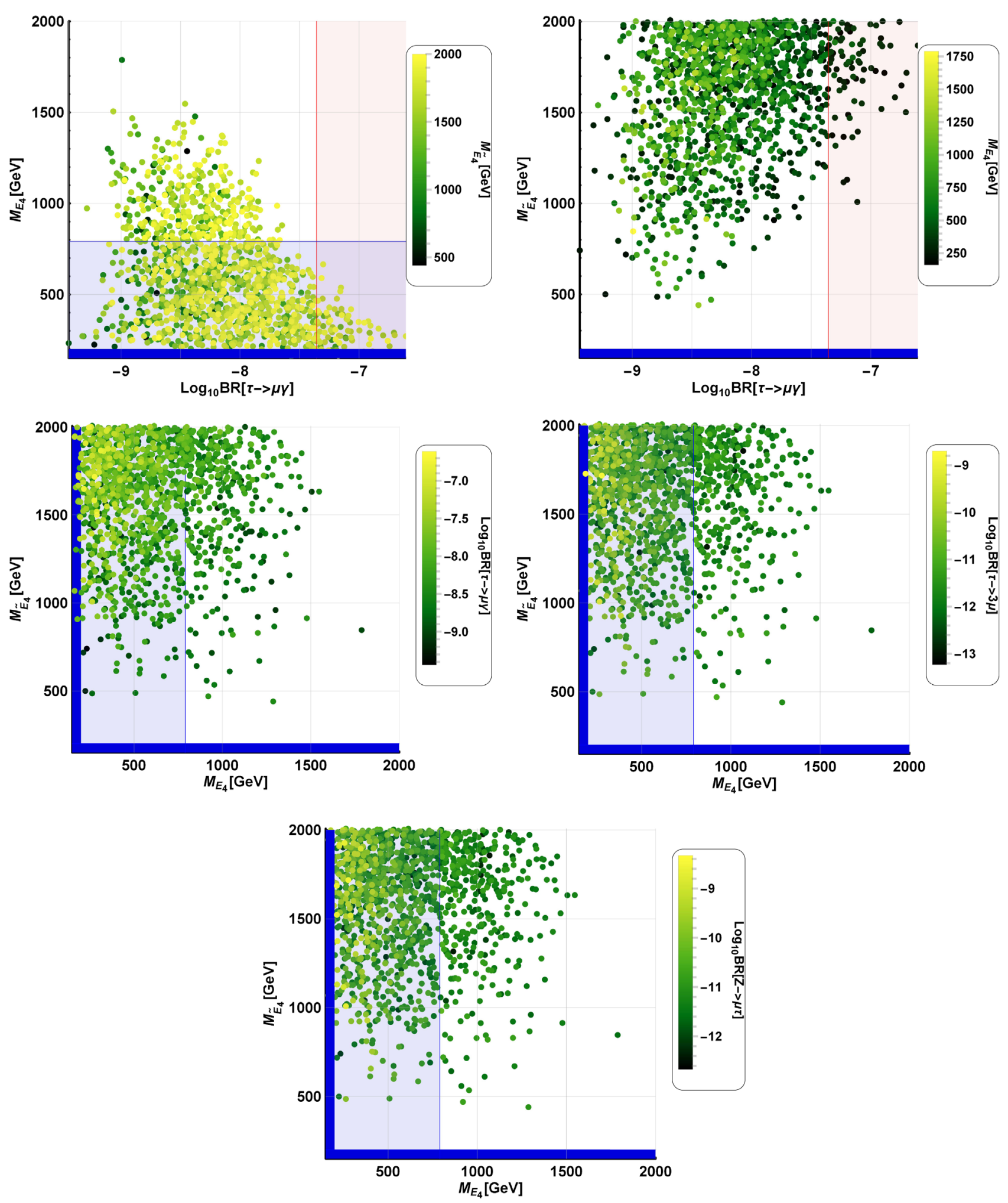

FIG. 8. Scanned mass region of the vectorlike charged leptons and contributions of the flavor violating interactions with the SM $Z$ gauge boson to the diverse CLFV decays $\tau \rightarrow \mu \gamma, \tau \rightarrow 3 \mu$ and $Z \rightarrow \mu \tau$. The used constraints are the predicted muon and tau mass to be put between [ $1 \pm 0.1] \times m_{\mu, \tau}$ and the 23 mixing angle to be less than 0.2 . The darker blue region appearing in each diagram means either the singlet or doublet vectorlike masses $M_{\tilde{E}_{4}}, M_{E_{4}}$ are excluded up to $200 \mathrm{GeV}$ by Ref. [17,18]. The brighter blue region means the doublet vectorlike mass $M_{E_{4}}$ is excluded up to $790 \mathrm{GeV}$ by the CMS $[19,20]$. The brighter red region appearing in above two plots is the region excluded by the experimental bound for the $\operatorname{BR}(\tau \rightarrow \mu \gamma)_{\mathrm{EXP}}=4.4 \times 10^{-8}$. 

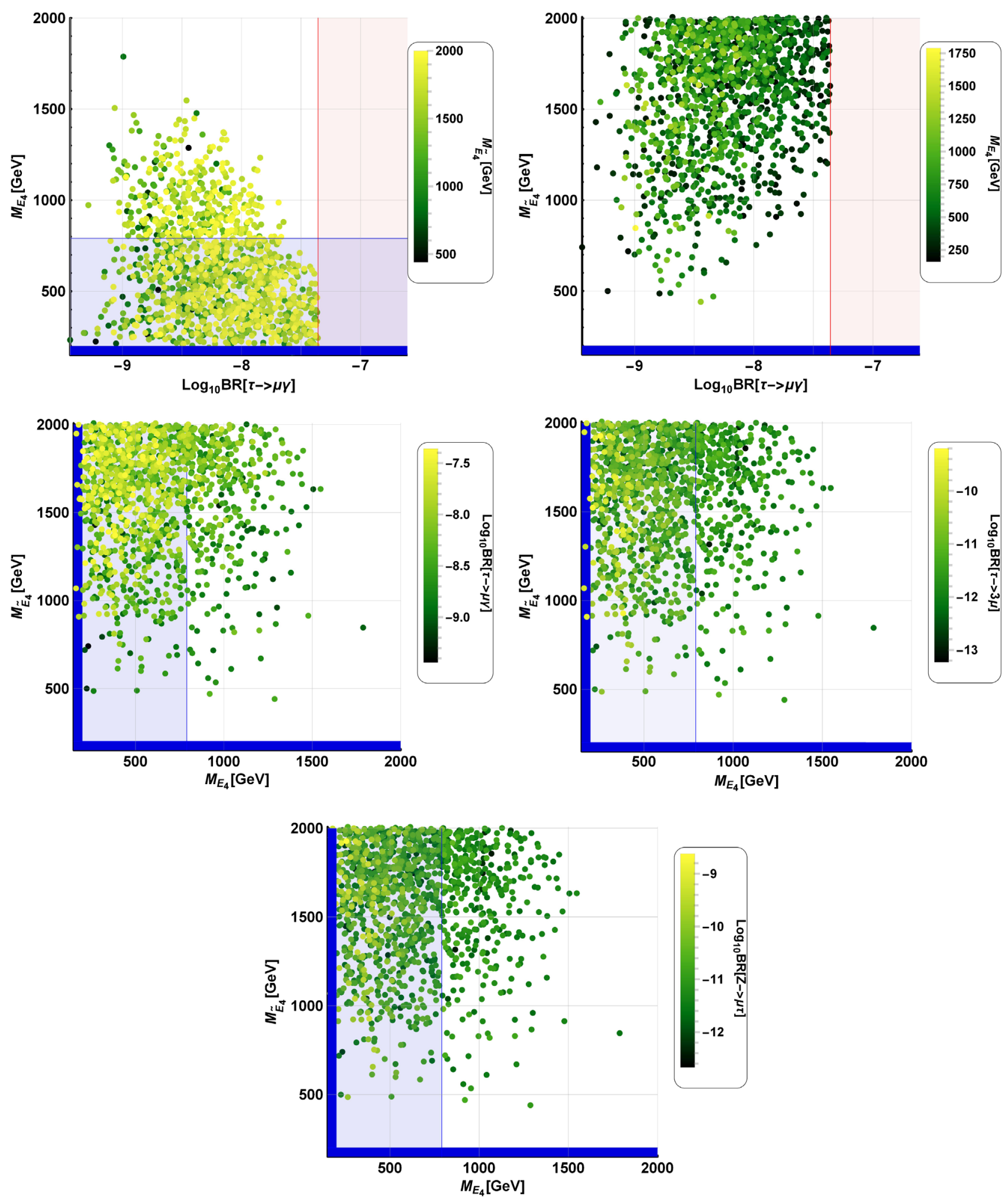

FIG. 9. Reduced number of numerical predictions. The numerical predictions are constrained by the experimental value of the branching ratio of $\tau \rightarrow \mu \gamma$ decay. None of them are constrained by the branching ratio of $\tau \rightarrow 3 \mu$ and $Z \rightarrow \mu \tau$ experimental bounds.

mass $M_{\tilde{E}_{4}}$ consists of the singlet vectorlike charged leptons $\tilde{E}_{4 L}, E_{4 R}$. Therefore, $M_{E_{4}}$ is the doublet vectorlike mass, whereas $M_{\tilde{E}_{4}}$ is the singlet vectorlike mass, and the doublet vectorlike mass is excluded by CMS up to $790 \mathrm{GeV}[19,20]$, expressed by the brighter blue region of Fig. 8. The second is our predictions for the branching ratio of $\tau \rightarrow \mu \gamma$ in Fig. 8 . The relevant experimental bound for each CLFV decay is given by: 


$$
\begin{aligned}
\mathrm{BR}(\tau \rightarrow \mu \gamma)_{\mathrm{EXP}}= & 4.4 \times 10^{-8} \\
& -\log _{10} \mathrm{BR}(\tau \rightarrow \mu \gamma)_{\mathrm{EXP}} \simeq-7.4 \\
\mathrm{BR}(\tau \rightarrow 3 \mu)_{\mathrm{EXP}}= & 2.1 \times 10^{-8} \\
& -\log _{10} \mathrm{BR}(\tau \rightarrow 3 \mu)_{\mathrm{EXP}} \simeq-7.7 \\
\mathrm{BR}(Z \rightarrow \mu \tau)_{\mathrm{EXP}}= & 1.2 \times 10^{-5} \\
& -\log _{10} \mathrm{BR}(Z \rightarrow \mu \tau)_{\mathrm{EXP}} \simeq-4.9 .
\end{aligned}
$$

Our predictions for the CLFV $\tau \rightarrow 3 \mu$ and $Z \rightarrow \mu \tau$ decays are not excluded by the experimental bound, however those are not the case for the CLFV decay $\tau \rightarrow \mu \gamma$, which exceed its upper experimental bound in some parts of the parameter space. This is due to, in some parts of the parameter space, the dominant contributions to the $\tau \rightarrow \mu \gamma$ decay involving a charged exotic lepton as well as chirality flip in the internal line and proportional to $M / m_{\tau}$ because of the sizeable large value of the charged exotic lepton-SM charged lepton mass ratio. After removing all excluded points by the experimental bound of the $\tau \rightarrow \mu \gamma$ decay, we obtain Fig. 9.

Looking at our numerical predictions for the branching ratio of the $\tau \rightarrow \mu \gamma$ decay shown in Fig. 9, some of them are constrained by the experimental limit of this branching ratio, however most of them survive, which implies that our numerical predictions for the branching ratio of $\tau \rightarrow \mu \gamma$ are not significantly constrained by its experimental bound. Furthermore, none of the numerical predictions for the branching ratios of the $\tau \rightarrow 3 \mu$ and $Z \rightarrow \mu \tau$ decays are constrained by its experimental bound. However, our numerical predictions for the different CLFV decays can significantly be constrained by the future LHC upgrades having higher center of mass energy and luminosity than the ones of the current LHC, which will allow to set tightest constraints on charged exotic vectorlike masses, thus leading to stronger constraints on the model parameter space. Regarding the CLFV $Z \rightarrow \mu \tau$ decay, the FCC-ee experiment has planned to generate $10^{12} \mathrm{SM} Z$ gauge bosons, which will allow to probe our model since the branching ratio of the $Z \rightarrow \mu \tau$ decay can reach values of the order of $10^{-10}$ in the allowed region of the parameter space. Thus, the $Z \rightarrow \mu \tau$ decay is within the reach of the FCC-ee experiment, whose $Z$ factory $[21,22]$ will be crucial to verify or rule out this model. Concluding this subsection, our numerical predictions are not significantly constrained by any of the CLFV $\tau \rightarrow \mu \gamma$, $\tau \rightarrow 3 \mu$ and $Z \rightarrow \mu \tau$ decays and might be able to be seen from the $Z$ factory for the first time, predicting the doublet vectorlike charged lepton mass which is ranged from 790 to $1600 \mathrm{GeV}$ whereas the singlet vectorlike charged lepton mass is ranged from 500 to $2000 \mathrm{GeV}$ or above than that.

\section{QUARK SECTOR PHENOMENOLOGY}

We have discussed the up- and down-type quark mass matrices pointed out that they have a different form, since the quark doublet rotation used in the up-type quark sector cannot remove the down-type Yukawa term. This difference between up- and down-type quark mass matrices cause a distinct feature for each sector as follows:

(i) The up-type quark mass matrix can reach to the 23 left (right)-handed mixing.

(ii) The down-type quark mass matrix can access to all left-handed among the three SM generations, whereas the right-handed mixing can only have the 23 mixing.

The interesting feature of the down-type quark mass matrix allows for flavor changing $Z$ interactions with down type quarks which yield neutral meson oscillations such as $K, B_{d}$, and $B_{s}$. Furthermore, an important feature to be mentioned is that the first generation of SM charged fermions do not acquire masses. Due to this property, our predictions for the neutral meson oscillations including the $d$ quark give a very suppressed energy difference corresponding to $10^{-40} \mathrm{GeV}$, which is impossible to reach with the current experimental sensitivity. Then, the rest of the neutral meson oscillation $B_{s}$ is possible and an encouraging feature of the $B_{s}$ meson oscillation in our proposed model is the $B_{s}$ meson oscillation mediated by the SM $Z$ gauge boson can be calculated at treelevel as given in Fig. 10.
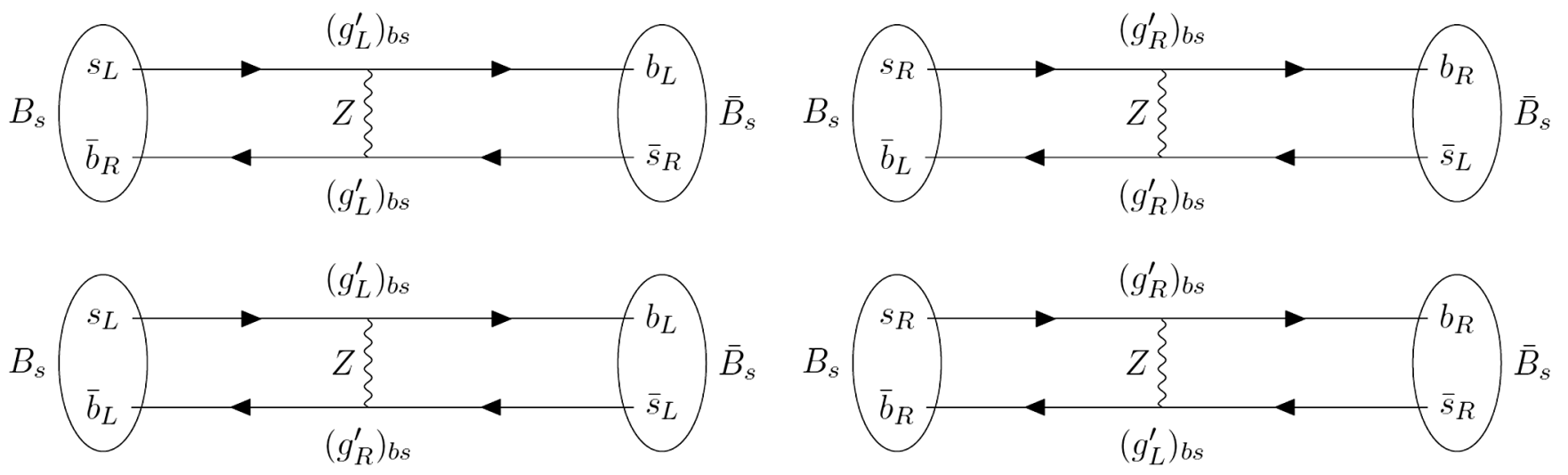

FIG. 10. Feynman diagrams contributing to the $B_{s}-\bar{B}_{s}$ meson mixing involving the tree-level exchange of the $Z$ gauge boson in the polarized basis. 
From our numerical analysis we have found that the BSM contributions to the $B_{s}$ meson oscillation arising from the tree-level exchange of the $Z$ gauge boson yield the meson mass splitting of the order of $10^{-15} \mathrm{GeV}$ or even less than that value, which is quite negligible compared to its corresponding experimental bound of $10^{-11} \mathrm{GeV}$. The very suppressed new physics effect for the $B_{s}$ meson oscillation can be explained by considering the flavor violating coupling constants at each vertex of each diagram, whose value is about $10^{-6,-7}$ and this values are determined by the ratio between Yukawa and vectorlike masses. For this reason, in the study of the phenomenological implications of our model in the flavor changing neutral interactions in the quark sector, we do not consider the neutral meson oscillations as well as the $B_{s} \rightarrow \mu^{+} \mu^{-}$decay. It is worth mentioning that the $B_{s} \rightarrow$ $\mu^{+} \mu^{-}$decay gives weaker effects than the neutral meson oscillations. Considering these facts, we conclude that the rare $t \rightarrow c Z$ decay and the CKM mixing matrix can constrain the quark sector of our model, and thus we discuss these two phenomenological aspects in the following subsections.

\section{A. Analytic expression for the $t \rightarrow c Z$ decay}

The $t \rightarrow c Z$ decay, which only appears at one-loop level in the SM, can take place at tree-level in our proposed model, thanks to the $Z$ mediated flavor changing neutral current interactions in the quark sector. In our proposed model, the $t \rightarrow c Z$ receives tree-level contributions which are depicted in Fig. 11.

Denoting the invariant amplitudes for the Feynman diagrams of the left and right panels of Fig. 11 as $\mathcal{M}_{L}$ and $\mathcal{M}_{R}$, respectively, we find that they can be written as:

$$
\begin{aligned}
i \mathcal{M}_{L} & =i\left(g_{L}\right)_{t c} \epsilon_{\mu}^{*}\left(p_{3}\right) \bar{u}\left(p_{2}\right) \gamma^{\mu} P_{L} u\left(p_{1}\right) \\
i \mathcal{M}_{R} & =i\left(g_{R}\right)_{t c} \epsilon_{\mu}^{*}\left(p_{3}\right) \bar{u}\left(p_{2}\right) \gamma^{\mu} P_{R} u\left(p_{1}\right) .
\end{aligned}
$$

In order to have the squared amplitude averaged and summed, we square the amplitudes given in Eq. (49) and then sum over the different spin states, as follows:

$$
\begin{aligned}
& \frac{1}{2} \sum_{\text {spin }}\left|\mathcal{M}_{L}\right|^{2}=\left(g_{L}\right)_{c t}^{2}\left(\left(p_{2} \cdot p_{1}\right)+\frac{2}{M_{Z}^{2}}\left(p_{2} \cdot p_{3}\right)\left(p_{1} \cdot p_{3}\right)\right) \\
& \frac{1}{2} \sum_{\text {spin }}\left|\mathcal{M}_{R}\right|^{2}=\left(g_{R}\right)_{c t}^{2}\left(\left(p_{2} \cdot p_{1}\right)+\frac{2}{M_{Z}^{2}}\left(p_{2} \cdot p_{3}\right)\left(p_{1} \cdot p_{3}\right)\right) \\
& \frac{1}{2} \sum_{\text {spin }}|\mathcal{M}|^{2}=\frac{1}{2} \sum_{\text {spin }}\left(\left|\mathcal{M}_{L}\right|^{2}+\left|\mathcal{M}_{R}\right|^{2}\right)=\frac{1}{2}\left(\left(g_{L}\right)_{c t}^{2}+\left(g_{R}\right)_{c t}^{2}\right)\left(\left(p_{2} \cdot p_{1}\right)+\frac{2}{M_{Z}^{2}}\left(p_{2} \cdot p_{3}\right)\left(p_{1} \cdot p_{3}\right)\right) .
\end{aligned}
$$

Then, the decay rate equation is given by:

$$
\begin{aligned}
d \Gamma(t \rightarrow c Z)= & \frac{1}{2 m_{t}} \frac{d^{3} p_{2}}{(2 \pi)^{3} 2 E_{2}} \frac{d^{3} p_{3}}{(2 \pi)^{3} 2 E_{3}}|\mathcal{M}|^{2}(2 \pi)^{4} \delta^{(4)}\left(p_{1}-p_{2}-p_{3}\right) \\
\Gamma(t \rightarrow c Z)= & \frac{\left|p^{*}\right|}{32 \pi^{2} m_{t}^{2}} \int|\mathcal{M}|^{2} d \Omega \\
= & \frac{1}{8 \pi m_{t}^{2}} \frac{1}{2 m_{t}}\left(m_{t}^{2}-M_{Z}^{2}\right)\left(\left(g_{L}\right)_{c t}^{2}+\left(g_{R}\right)_{c t}^{2}\right) \\
& \times\left[\frac{m_{t}^{2}+m_{c}^{2}-M_{Z}^{2}}{2}+\frac{2}{M_{Z}^{2}}\left(\frac{m_{t}^{2}-m_{c}^{2}-M_{Z}^{2}}{2}\right)\left(\frac{m_{t}^{2}-m_{c}^{2}+M_{Z}^{2}}{2}\right)\right]
\end{aligned}
$$

where $p^{*} \simeq \frac{1}{2 m_{t}}\left(m_{t}^{2}-M_{Z}^{2}\right)$. Then, we are ready to write down our prediction for the branching ratio of the tree-level $t \rightarrow c Z$ decay [23]:
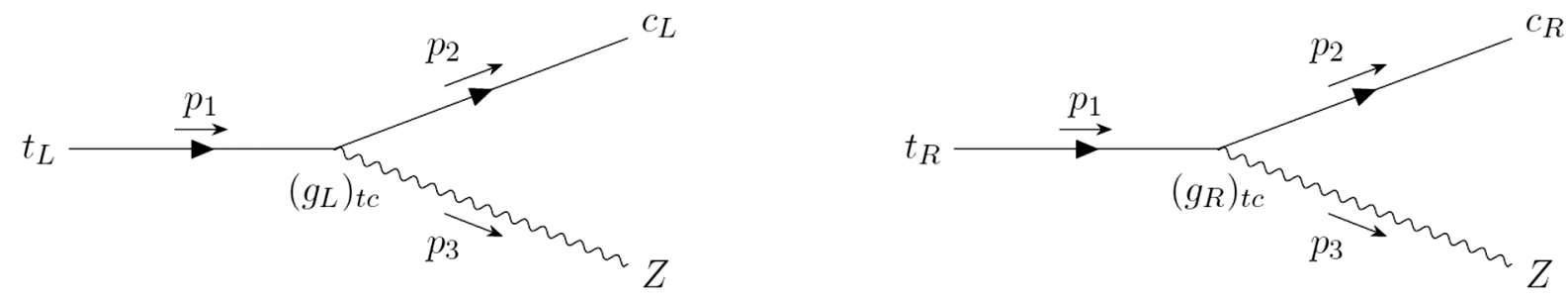

FIG. 11. tree-level Feynman diagrams contributing to the rare $t \rightarrow c Z$ decay. 


$$
\mathrm{BR}(t \rightarrow c Z)=\frac{\Gamma(t \rightarrow c Z)}{\Gamma_{t}}<\mathrm{BR}(t \rightarrow c Z)_{\mathrm{EXP}}=2.4 \times 10^{-4} \quad(95 \% \mathrm{CL})
$$

where $\Gamma_{t}=1.32 \mathrm{GeV}$.

\section{B. Analytic expression for the CKM mixing matrix}

In order to discuss the CKM mixing matrix, the first task we need to investigate is the $W$ current of the SM in order to see how the CKM mixing matrix can take place (we only consider the three SM generations at the moment).

$$
\begin{aligned}
\mathcal{L}_{\mathrm{SM}}^{W} & =g j_{\mu}^{W+} W^{\mu+}=\frac{g}{\sqrt{2}}\left(\bar{u}_{L}^{i} \gamma_{\mu} d_{L}^{i}\right) W^{\mu+} \\
& =\frac{g}{\sqrt{2}}\left(\bar{u}_{L}^{i}\left(V_{L}^{u \dagger} V_{L}^{u}\right) \gamma_{\mu}\left(V_{L}^{d^{\dagger}} V_{L}^{d}\right) d_{L}^{i}\right) W^{\mu+}=\frac{g}{\sqrt{2}}\left(\bar{u}_{L}^{i l} \gamma_{\mu}\left(V_{\mathrm{CKM}}\right) d_{L}^{i \prime}\right) W^{\mu+}
\end{aligned}
$$

where $u_{L}^{i \prime}, d_{L}^{i \prime}$ are the up- and down-type quarks of the SM in the mass basis and $V_{\mathrm{CKM}}$ is the CKM mixing matrix defined as $V_{L}^{u} V_{L}^{d \dagger}$. Now we extend the quark spectrum by considering the vectorlike quarks, thus implying that the $W$ current takes the form:

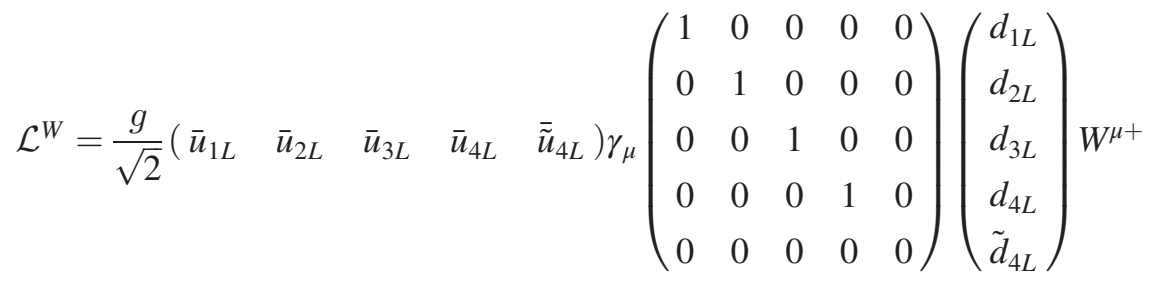

$$
\begin{aligned}
& =\frac{g}{\sqrt{2}}\left(\begin{array}{lllll}
\bar{u}_{1 L} & \bar{u}_{2 L} & \bar{u}_{3 L} & \bar{u}_{4 L} & \overline{\tilde{u}}_{4 L}
\end{array}\right) V_{L}^{u \dagger} V_{L}^{u} \gamma_{\mu}\left(\begin{array}{ccccc}
1 & 0 & 0 & 0 & 0 \\
0 & 1 & 0 & 0 & 0 \\
0 & 0 & 1 & 0 & 0 \\
0 & 0 & 0 & 1 & 0 \\
0 & 0 & 0 & 0 & 0
\end{array}\right) V_{L}^{d^{\dagger}} V_{L}^{d}\left(\begin{array}{c}
d_{1 L} \\
d_{2 L} \\
d_{3 L} \\
d_{4 L} \\
\tilde{d}_{4 L}
\end{array}\right) W^{\mu+}
\end{aligned}
$$

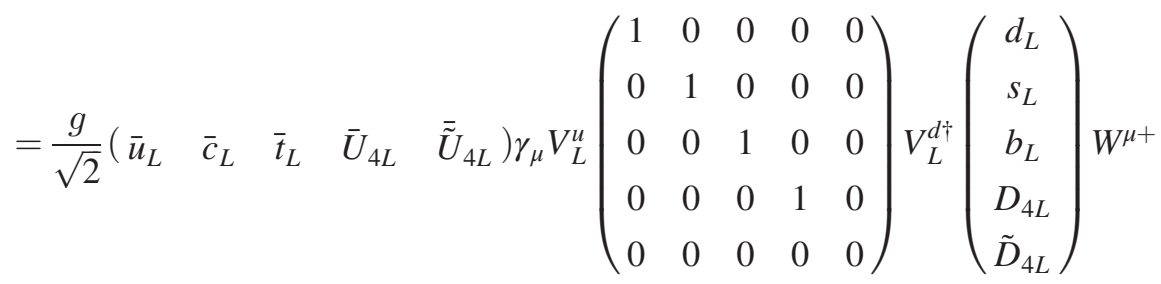

where the CKM mixing prediction in our model is given by

$$
V_{\mathrm{CKM}}=V_{L}^{u}\left(\begin{array}{ccccc}
1 & 0 & 0 & 0 & 0 \\
0 & 1 & 0 & 0 & 0 \\
0 & 0 & 1 & 0 & 0 \\
0 & 0 & 0 & 1 & 0 \\
0 & 0 & 0 & 0 & 0
\end{array}\right) V_{L}^{d \dagger}, \quad \text { for the upper-left } 3 \times 3 \text { block }
$$

where $V_{L}^{u}$ is the mixing matrix for the up-type quarks defined in Eq. (13) and $V_{L}^{d}$ is the one corresponding to the down-type quarks given in Eq. (15). The zero appearing in the middle matrix between $V_{L}^{u}$ and $V_{L}^{d \dagger}$ arises from the left-handed vectorlike quark singlets $\tilde{U}_{4 L}$ and $\tilde{D}_{4 L}$ which do not interact with the $W$ currents, so our prediction for the CKM mixing matrix does not feature the unitarity requirement and this leads to the need of relaxing the unitarity constraint of the CKM quark mixing matrix. That deviation of unitarity of the CKM quark mixing matrix is due to the presence of heavy vectorlike 
quarks and this aspect was studied in [24] in the context of theory with different particle spectrum and symmetry than ours. Few sigma of SM deviations from the first row of the CKM mixing matrix without unitarity were analyzed in [24]. The deviation from the Unitarity of the CKM will also be discussed in our numerical result and the experimental CKM mixing matrix without unitarity is given by $[24,25]$ :

$$
\left|K_{\mathrm{CKM}}\right|=\left(\begin{array}{lll}
0.97370 \pm 0.00014 & 0.22450 \pm 0.00080 & 0.00382 \pm 0.00024 \\
0.22100 \pm 0.00400 & 0.98700 \pm 0.01100 & 0.04100 \pm 0.00140 \\
0.00800 \pm 0.00030 & 0.03880 \pm 0.00110 & 1.01300 \pm 0.03000
\end{array}\right)
$$

\section{Numerical analysis for each prediction in the quark sector}

When compared to the charged lepton sector simulation, the quark sector becomes much more complicated since we need to fit the masses of the $c, t, s$, and $b$ quarks simultaneously as well as the CKM mixing matrix without imposing the unitarity requirement. Therefore, we fit the masses of the four quarks first by using a fitting function $\chi_{\text {mass }}^{2}$ and then we start a second fitting procedure by using another fitting function $\chi_{\text {CKM }}^{2}$ and this will be discussed in detail in the following subsections.

\section{The fitting function $\chi^{2}$ and free parameter setup}

We set up our parameter region as follows:

There are a few things to be noticed as in the charged lepton case.

(1) The relation $v_{u}^{2}+v_{d}^{2}=(246 \mathrm{GeV})^{2}$ still holds and the mass parameters $m_{24,34,43}^{u}$ cannot exceed the upper perturbative limit on the Yukawa constant $\sqrt{4 \pi} \simeq 3.54$ multiplied by the vev $\approx 240 \mathrm{GeV}$, of the $H_{u}$ Higgs, thus yielding the bound of $850 \mathrm{GeV}$ for these mass parameters. These restrictions have been taken into account through the whole fitting process.

(2) The down-type Higgs $H_{d}$ has a very small vev, which is about order of $10 \mathrm{GeV}$, based on our previous analysis [4], and the range of values of the mass parameters $m_{14,24,34,43}^{d}$ are considered under this assumption.

(3) Since we do not know the correct scale of $v_{\phi}$, we considered $m_{35}^{Q}, m_{52,53}^{u}$ and $m_{52,53}^{d}$ as free parameters. For the same reason, the vectorlike masses $M_{45}^{Q}$ and $M_{54}^{u, d}$ are considered free parameters as well.

(4) The mass parameters $m_{35}^{Q}$ and $M_{45}^{Q}$ appear in a common term shared by both up- and down-type quark sector mass matrices and this feature has been discussed in the paragraph below Eq. (14).

The next thing to do is to set up the two fitting functions $\chi_{\text {mass }}^{2}$ and $\chi_{\text {CKM }}^{2}$ as follows:

$$
\begin{aligned}
\chi_{\text {mass }}^{2} & =\sum_{f=c, t, s, b}=\frac{\left(m_{f}^{\text {pred }}-m_{f}^{\mathrm{EXP}}\right)^{2}}{\left(\delta m_{f}^{\mathrm{EXP}}\right)^{2}}, \\
\chi_{\mathrm{CKM}}^{2} & =\sum_{i, j=1,2,3} \frac{\left(\left(V_{\mathrm{CKM}}^{\mathrm{pred}}\right)_{i j}-\left(V_{\mathrm{CKM}}^{\mathrm{EXP}}\right)_{i j}\right)^{2}}{\left(\left(\delta V_{\mathrm{CKM}}^{\mathrm{EXP}}\right)_{i j}\right)^{2}},
\end{aligned}
$$

where the superscript pred means our prediction to its experimental value and the delta means error bar of the physical quantity at $1 \sigma$. Our first goal is to fit the masses of the four quarks simultaneously. For the charged lepton case, we require that our obtained muon and tau masses to be in the range $[1 \pm 0.1] \times m_{\mu, \tau}$ and this requirement is also imposed for the $c, s$ and $b$ quarks excepting for the $t$ quark since the $t$ quark is too heavy. Besides that, we require that the obtained top quark mass to be in the range $[1 \pm 0.01] \times$ $m_{t}$ instead of $[1 \pm 0.1] \times m_{t}$. After the mass parameters have been converged to be put between the arranged range of each quark mass, we use the other fitting function $\chi_{\mathrm{CKM}}^{2}$ to fit our prediction for the CKM mixing matrix. Using one

TABLE III. Initial parameter setup for scanning mass of the vectorlike quarks.

\begin{tabular}{lc}
\hline \hline Mass parameter & Scanned Region $(\mathrm{GeV})$ \\
\hline$y_{24}^{u} v_{u}=m_{24}^{u}$ & $\pm[10,50]$ \\
$y_{34}^{u} v_{u}=m_{34}^{u}$ & $\pm[200,400]$ \\
$y_{43}^{u} v_{u}=m_{43}^{u}$ & $\pm[200,400]$ \\
$x_{34}^{Q} v_{\phi}=m_{35}^{Q}$ & $m_{35}^{Q}$ \\
$x_{42}^{u} v_{\phi}=m_{52}^{u}$ & $\pm[500,700]$ \\
$x_{43}^{u} v_{\phi}=m_{53}^{u}$ & $\pm[50,500]$ \\
$M_{45}^{Q}$ & $M_{45}^{Q}$ \\
$M_{54}^{u}$ & $\pm[1000,3000]$ \\
$y_{14}^{d} v_{d}=m_{14}^{d}$ & $\pm[1,10]$ \\
$y_{24}^{d} v_{d}=m_{24}^{d}$ & $\pm[5,20]$ \\
$y_{34}^{d} v_{d}=m_{34}^{d}$ & $\pm[10,30]$ \\
$y_{43}^{d} v_{d}=m_{43}^{d}$ & $\pm[5,10]$ \\
$x_{34}^{Q} v_{\phi}=m_{35}^{Q}$ & $\pm[10,100]$ \\
$x_{42}^{d} v_{\phi}=m_{52}^{d}$ & $\pm[10,100]$ \\
$x_{43}^{d} v_{\phi}=m_{53}^{d}$ & $\pm[10,100]$ \\
$M_{45}^{Q}$ & $\pm[1000,3000]$ \\
$M_{54}^{d}$ & $\pm[1000,3000]$ \\
\hline \hline
\end{tabular}


of the defined fitting functions, we need to vary the mass parameters of Table III by a factor of $[1 \pm \kappa]$ where $\kappa=0.1$ in order to find better mass parameters. We rename the given parameters from the initial parameter setup by adding an subscript $r$ to the mass parameters. The varied mass parameters are given in Table IV.

We vary the parameter space given in Table IV by first using the fitting function $\chi_{\text {mass }}^{2}$ in order to find a suitable mass prediction for the four quarks $t, b, c$, and $b$. Once the obtained masses of these quarks are allocated in the ranges $\left([1 \pm 0.1] \times m_{c, s, b}\right.$ and $\left.[1 \pm 0.01] \times m_{t}\right)$, we proceed to fit the CKM quark mixing matrix once more by using the other fitting function $\chi_{\text {CKM }}^{2}$ and it is worth mentioning that fitting the CKM mixing matrix is much more challenging due to the very small experimental errors of the CKM matrix elements. We display a benchmark point most converged for the CKM mixing matrix at the next subsection to discuss the possible deviation from the SM result arising from the first row of the CKM mixing matrix.

\section{Numerical scan result for the quark sector}

We start with the most converged benchmark point $\left(\chi_{\mathrm{CKM}}^{2}=956.828\right)$ after repeating the varying many times
TABLE IV. Next parameter setup after the initial parameter setup to find better mass parameters.

\begin{tabular}{lc}
\hline \hline Mass parameter & Scanned Region $(\mathrm{GeV})$ \\
\hline$y_{24}^{u} v_{u}=m_{24}^{u}$ & {$[1 \pm \kappa] \times m_{24 r}^{u}$} \\
$y_{34}^{u} v_{u}=m_{34}^{u}$ & {$[1 \pm \kappa] \times m_{34 r}^{u}$} \\
$y_{43}^{u} v_{u}=m_{43}^{u}$ & {$[1 \pm \kappa] \times m_{43 r}^{u}$} \\
$x_{34}^{Q} v_{\phi}=m_{35}^{Q}$ & $m_{35}^{Q}$ \\
$x_{42}^{u} v_{\phi}=m_{52}^{u}$ & {$[1 \pm \kappa] \times m_{42 r}^{u}$} \\
$x_{43}^{u} v_{\phi}=m_{53}^{u}$ & {$[1 \pm \kappa] \times m_{43 r}^{u}$} \\
$M_{45}^{Q}$ & $M_{45}^{Q}$ \\
$M_{54}^{u}$ & {$[1 \pm \kappa] \times M_{54 r}^{u}$} \\
$y_{14}^{d} v_{d}=m_{14}^{d}$ & {$[1 \pm \kappa] \times m_{14 r}^{d}$} \\
$y_{24}^{d} v_{d}=m_{24}^{d}$ & {$[1 \pm \kappa] \times m_{24 r}^{d}$} \\
$y_{34}^{d} v_{d}=m_{34}^{d}$ & {$[1 \pm \kappa] \times m_{34 r}^{d}$} \\
$y_{43}^{d} v_{d}=m_{43}^{d}$ & {$[1 \pm \kappa] \times m_{43 r}^{d}$} \\
$x_{34}^{Q} v_{\phi}=m_{35}^{Q}$ & {$[1 \pm \kappa] \times m_{35 r}^{Q}$} \\
$x_{42}^{d} v_{\phi}=m_{52}^{d}$ & {$[1 \pm \kappa] \times m_{42 r}^{d}$} \\
$x_{43}^{d} v_{\phi}=m_{53}^{d}$ & {$[1 \pm \kappa] \times m_{43 r}^{d}$} \\
$M_{45}^{Q}$ & {$[1 \pm \kappa] \times M_{45 r}^{Q}$} \\
$M_{54}^{d}$ & {$[1 \pm \kappa] \times M_{54 r}^{d}$} \\
$\kappa$ & 0.1 \\
\hline \hline
\end{tabular}

$$
\begin{aligned}
M^{u} & =\left(\begin{array}{llllc}
0 & 0 & 0 & 0 & 0 \\
0 & 0 & 0 & 0 & 14.474 \\
0 & 0 & 0 & 1206.340 & 277.563 \\
0 & 0 & 273.503 & -1775.200 & 0 \\
0 & 550.990 & 434.462 & 0 & -5624.050
\end{array}\right) \quad M^{d}=\left(\begin{array}{ccccc}
0 & 0 & 0 & 0 & -0.938 \\
0 & 0 & 0 & 0 & -4.041 \\
0 & 0 & 0 & 1206.340 & -27.427 \\
0 & 0 & -5.636 & -1775.200 & 0 \\
0 & 72.915 & -75.760 & 0 & 2623.620
\end{array}\right) \\
M_{\text {diag }}^{u}= & \left(\begin{array}{ccccc}
0 & 0 & 0 & 0 & 0 \\
0 & 1.255 & 0 & 0 & 0 \\
0 & 0 & 171.303 & 0 & 0 \\
0 & 0 & 0 & 2155.890 & 0 \\
0 & 0 & 0 & 0 & 5674.840
\end{array}\right) \quad M_{\text {diag }}^{d}=\left(\begin{array}{cccccc}
0 & 0 & 0 & 0 & 0 \\
0 & 0.094 & 0 & 0 & 0 \\
0 & 0 & 3.875 & 0 & 0 \\
0 & 0 & 0 & 2146.190 & 0 \\
0 & 0 & 0 & 0 & 2625.960
\end{array}\right),
\end{aligned}
$$

where the above two mass matrices of Eq. (58) are the mass matrices for the up- and down-type quarks in the flavor basis, whereas the below two mass matrices are ones fully diagonalized, so revealing all propagating quark mass. From the mass matrices of Eq. (58), we have the mixing matrices $V_{L}^{u}$ and $V_{L}^{d}$ and arrive to our CKM prediction using the formula of Eq. (55).

$$
V_{\text {CKM }}^{\text {pred }}=\left(\begin{array}{ccccc}
0.97409 & 0.22602 & 0.00799 & -6.38471 \times 10^{-6} & -0.00036 \\
0.22615 & -0.97372 & -0.02697 & 3.80102 \times 10^{-5} & 0.00147 \\
0.00166 & 0.02815 & -0.99880 & -0.00766 & 0.00874 \\
0.00003 & 0.00019 & -0.00916 & 0.99919 & -0.01773 \\
-0.00057 & 0.00112 & 0.03812 & 0.03539 & -0.00096
\end{array}\right)
$$

where a feature we should remember is the left-handed down-quark sector are able to reach to all mixings among the three SM generations, whereas the only left-handed 23 mixing is allowed for the up-quark sector in this BSM model. The experimental CKM mixing matrix without unitarity is given in Eq. (60). 


$$
\left|K_{\mathrm{CKM}}\right|=\left(\begin{array}{lll}
0.97370 \pm 0.00014 & 0.22450 \pm 0.00080 & 0.00382 \pm 0.00024 \\
0.22100 \pm 0.00400 & 0.98700 \pm 0.01100 & 0.04100 \pm 0.00140 \\
0.00800 \pm 0.00030 & 0.03880 \pm 0.00110 & 1.01300 \pm 0.03000
\end{array}\right)
$$

Restricting our attention to the upper-left $3 \times 3$ block of Eq. (59), it can be compared to its experimental bound given in Eq. (60). In order to confirm that our prediction for the CKM mixing matrix is consistent with the experimental data, it requires for the upper-left $3 \times 3$ block of Eq. (59) to be inside the $3 \sigma$ experimentally allowed range as follows:

$$
\left|\left(K_{\mathrm{CKM}}\right)_{i j}\right|-3\left|\left(\delta K_{\mathrm{CKM}}\right)_{i j}\right|<\left|\left(V_{\mathrm{CKM}}^{\mathrm{pred}}\right)_{i j}\right|<\left|\left(K_{\mathrm{CKM}}\right)_{i j}\right|+3\left|\left(\delta K_{\mathrm{CKM}}\right)_{i j},\right|, \quad \text { for } i, j=1,2,3
$$

and we confirm that the $13,23,31,32$ elements in the CKM prediction of Eq. (59) cannot be fitted within the $3 \sigma$ range with a small difference. From our numerical analysis we find that in our model the CKM quark mixing matrix mainly arises from the down type quark sector and has a subleading correction coming from the up type quark sector. It is worth mentioning that the inclusion of an additional vectorlike family in our proposed model to provide masses for the first generation of SM charged fermions will lead to an improvement of our predictions related to the CKM quark mixing matrix. However, that approach of having a fifth vectorlike fermion family goes beyond the scope in this work and is deferred for a future publication. Furthermore, in this section we also discuss the possible deviation of the first row of the CKM mixing matrix without unitarity and this study is also covered in this Ref. [24,26] with an isosinglet vectorlike quark in a model different than the one considered in this paper. According to $[24,26]$, the deviation $\Delta$ of unitarity is defined as follows:

$$
\Delta=1-\left|V_{u d}\right|^{2}-\left|V_{u s}\right|^{2}-\left|V_{u b}\right|^{2}
$$

and its experimental value is given by [27].

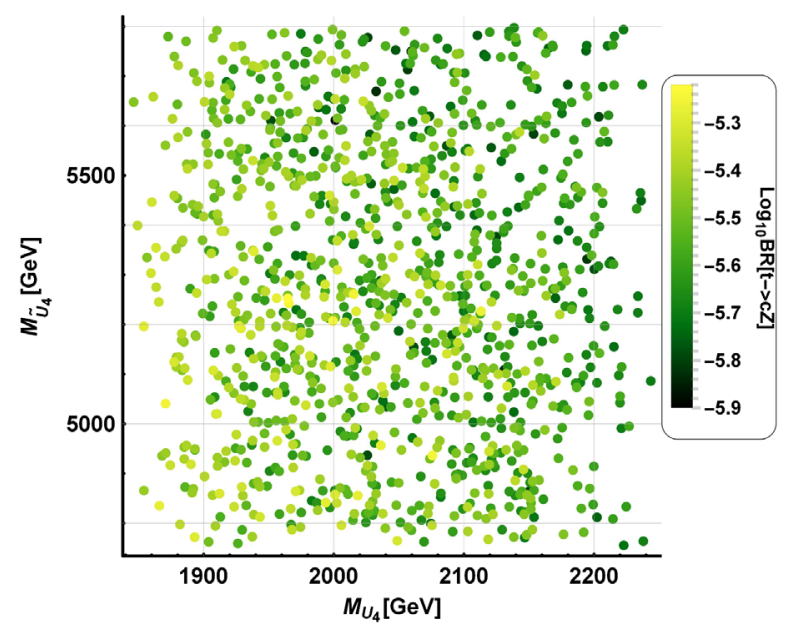

$$
\sqrt{\Delta} \sim 0.04
$$

Calculating the deviation of unitarity $\Delta$ from the best fitted CKM prediction of Eq. (59), the result is

$$
\sqrt{\Delta} \simeq 0.00035 \text {. }
$$

Therefore, the deviation of unitarity derived from the model under consideration is too small to be observed compared to its experimental bound given in Eq. (63). Lastly, we discuss the rare $t \rightarrow c Z$ decay and collect all benchmark points satisfying $\chi_{\text {CKM }}^{2}<980$ (notice that the most converged point reports $\chi_{\text {CKM }}^{2}=956.828$ ).

Figure 12 displays the allowed values of vectorlike quark masses consistent with the constraints arising from the rare $t \rightarrow c Z$ decay. Our obtained values for the vectorlike quark masses are consistent with their lower experimental bound of $1000 \mathrm{GeV}$ arising from collider searches. In our numerical analysis the vectorlike doublet up-type quark mass $M_{U_{4}}$ is ranged from $1850 \mathrm{GeV}$ up to about $2250 \mathrm{GeV}$ and the vectorlike singlet up-type quark mass $M_{\tilde{U}_{4}}$ is varied from $4750 \mathrm{GeV}$ to $5800 \mathrm{GeV}$. Regarding the exotic down

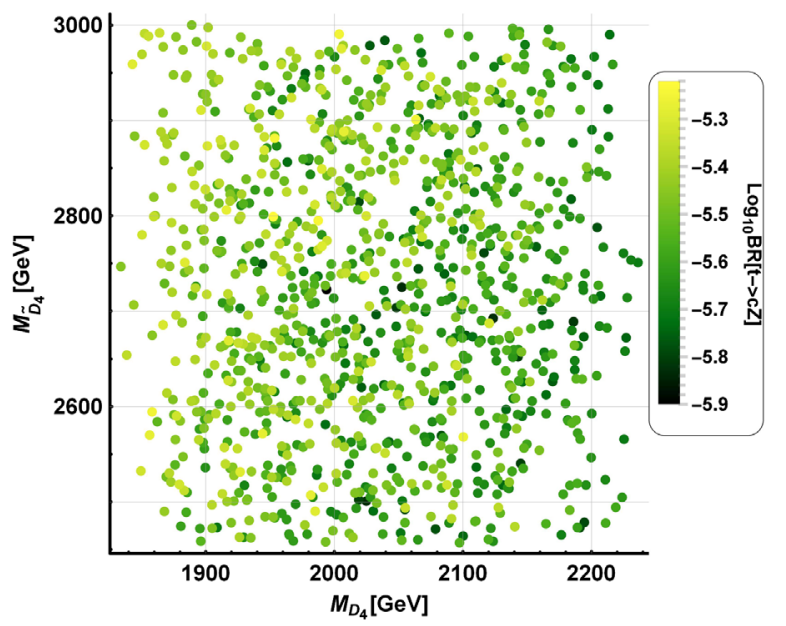

FIG. 12. Scanned mass region of the vectorlike quarks and contributions of the flavor violating interactions with the SM $Z$ gauge boson to the rare $t \rightarrow c Z$ decay. The used constraints are the predicted $c, s, b$, and $t$ quark mass to be put between $[1 \pm 0.1] \times m_{c, s, b}$ and $[1 \pm 0.01] \times m_{t}$ and the CKM mixing matrix. 
type quark sector, we have varied the vectorlike doublet down-type quark mass $M_{D_{4}}$ from $1850 \mathrm{GeV}$ up to $2250 \mathrm{GeV}$ and the vectorlike singlet down-type quark mass $M_{\tilde{D}_{4}}$ from $2450 \mathrm{GeV}$ up to $3000 \mathrm{GeV}$. As seen from Fig. 12 the order of magnitude of the obtained values for the branching ratio of the rare $t \rightarrow c Z$ decay range from $10^{-6}$ up to $10^{-5}$, which is consistent with its current experimental bound, whose logarithmic value is about -3.6 as indicated by Eq. (52).

\section{CONCLUSION}

In this work we have considered a model where the SM fermion sector is extended by the inclusion of a fourth vectorlike family and the scalar sector is augmented by the incorporation of an extra scalar doublet and a gauge singlet scalar. In addition, we have assumed a global $U(1)^{\prime}$ symmetry under which all particles are charged except the SM chiral quark and lepton fields. The model explains the hierarchical structure of the SM quark and lepton masses by assuming that the SM Yukawa interactions are forbidden by the $U(1)^{\prime}$ symmetry and arise effectively after it is spontaneously broken, due to induced mixing with the fourth vectorlike family. This mixing also results in nonstandard couplings of the $W$ and $Z$ gauge bosons which have been studied here for the first time.

This setup leads to sizeable branching fractions for the FCNC decays such as $\mu \rightarrow e \gamma, Z \rightarrow \mu \tau$ and $t \rightarrow c Z$, within the reach of the future experimental sensitivity. These FCNC decays are studied in detail in this work, in order to set constraints on the model parameter space. A great advantage of the approach taken in this work with respect to the ones considered in extensions of the SM having a $Z^{\prime}$ gauge boson is that it makes the study of the FCNC observables simpler than in the latter since in the former we can avoid assuming specific values for the unknown $U(1)^{\prime}$ coupling and $Z^{\prime}$ gauge boson mass. This makes the present phenomenology based on $W$ and $Z$ gauge boson couplings more predictive than if the $U(1)^{\prime}$ were a spontaneously broken gauge symmetry, leading to a massive $Z^{\prime}$.

Given that the hierarchical structure of the SM is implemented in our proposed model, the extended mass matrices for the charged lepton and quark sectors need to be completely and accurately diagonalized, as the starting point of our analytical and numerical analysis. Since we only consider a fourth vectorlike family, the model cannot provide masses for the first generation of SM charged fermions, nevertheless this is a good approximation given that the first generation of the SM fermions are very light. For this reason, we mainly focus on the study of FCNC observables involving the second and third generations of SM fermions in both quark and lepton sectors.

In the chosen convenient basis, the different shape of the down-type quark mass matrix allows all left-handed mixings between the three SM generations, whereas the up type quark sector can have only the 23 left-handed mixing, while all quarks and charged lepton have the only 23 righthanded mixing, and we have checked that the results are basis independent. This feature implies that we can obtain a prediction for the CKM mixing matrix and this is one of main phenomenological aspects analyzed in this work. In order to diagonalize the fermionic mass matrices, in an analytic approximation, we have defined the $S U(2)$ conserving and $S U(2)$ violating mixings and we have shown that the $S U(2)$ violating mixing plays a crucial role for generating the $Z$ mediated flavor violating interactions. Furthermore, the extension of the SM fermion sector by the inclusion of a vectorlike family makes the matrices of $Z$ couplings with fermions different than the identity matrix due to the appearance of nonzero off-diagonal matrix elements of the $Z$ coupling matrices which will give rise to flavor violating $Z$ decays. The nonzero off-diagonal SM $Z$ gauge coupling constants are generally proportional to two of the small mixing angles, which are defined by the ratio between the SM fermion and vectorlike masses, thus leading to small values. Defining all the required $Z$ gauge coupling constants with fermions in the mass basis, as discussed above, we began by analyzing the FCNC processes of the charged lepton sector. We have found that in the lepton sector, the following three FCNC decays are allowed: $\tau \rightarrow \mu \gamma, \tau \rightarrow 3 \mu$ and $Z \rightarrow \mu \tau$. Regarding the $\tau \rightarrow \mu \gamma$ decay, we discussed its leading contribution, which arises from the Feynman diagrams having a chirality flip in the internal fermionic lines and being proportional to $M / m_{\tau}$, where $M$ is the mass scale of the heavy charged vectorlike leptons. However, the dominant terms cannot be as big as the vectorlike masses get heavier since their coupling constants get suppressed at the same time, thus providing a balanced relation between the vectorlike masses and their coupling constants. We have found that our predictions for the vectorlike charged lepton masses are not severely constrained by the $\tau \rightarrow \mu \gamma$ decay since most of the obtained values for the $\tau \rightarrow \mu \gamma$ decay are consistent with its experimental upper bound. In the concerning to the $\tau \rightarrow 3 \mu$ and $Z \rightarrow \mu \tau$ decays, we have derived an analytic expression for their corresponding rates at tree-level finding that none of our predictions is constrained by the experimental bounds of these decays. Considering the FCC-ee experiment which have planned to generate $10^{12}$ the $Z$ gauge bosons and our numerical prediction for the $Z \rightarrow \mu \tau$ branching ratio is of the order of $10^{-9}$ at most, thus implying that our model can be tested at the $Z$ factory via the $Z \rightarrow \mu \tau$ decay. However, the CMS provided that the doublet vectorlike mass can be constrained up to $790 \mathrm{GeV}$ $[4,17]$ and our numerical predictions for the vectorlike charged leptons are severely constrained by the CMS result. Therefore, we can expect that the vectorlike charged lepton doublet mass is ranged from 790 to nearly $1600 \mathrm{GeV}$, whereas the vectorlike charged lepton singlet mass is ranged from 500 to $2000 \mathrm{GeV}$ or above than that. 
Turning to the quark sector phenomenology, we have analyzed the rare $t \rightarrow c Z$ decay as well as the CKM mixing to set constraints on the quark sector parameters. It is worth mentioning that the neutral meson $K, B_{d}, B_{s}$ oscillations do not set constraints on the quark sector parameters of our model since their new physics effects are quite negligible compared to the SM expectation. We have derived analytic expressions for the rare $t \rightarrow c Z$ decay as well as for the CKM mixing matrix. Due to the mixings between SM fermions and vectorlike fermions, the CKM quark mixing matrix is not unitary, thus implying that the unitarity requirement has to be relaxed $[24,25]$. Using the most converged benchmark point, we showed how dominant the down-type quark mixing matrix plays a crucial role in the CKM mixing matrix and we discussed the deviation of unitarity arisen from the first row of the CKM mixing matrix, whose value is too small to be experimentally measured. Finally, we investigate the branching ratio for the $t \rightarrow c Z$ decay and found that our numerical predictions are not excluded by its experimental bound, for vectorlike doublet up-type and down type quark masses $M_{U_{4}}$ and $M_{D_{4}}$ in the window $1850 \mathrm{GeV} \leq M_{U_{4}}, M_{D_{4}} \leq 2250 \mathrm{GeV}$ as well as vectorlike singlet up and down type quark masses $M_{\tilde{U}_{4}}$ and $M_{\tilde{D}_{4}}$ in the ranges $4750 \mathrm{GeV} \leq M_{\tilde{U}_{4}} \leq 5800 \mathrm{GeV}$, and $2450 \mathrm{GeV} \leq M_{\tilde{D}_{4}} \leq 3000 \mathrm{GeV}$, respectively.

In conclusion, we have analyzed a range of FCNCs arising from nonstandard $W$ and $Z$ gauge boson couplings in an extension of the SM with a fourth vectorlike family, which can also address the hierarchy of quark and lepton masses, leading to several interesting rare decays which may be probed in future high luminosity experiments.

\section{ACKNOWLEDGMENTS}

This research has received funding from Fondecyt (Chile), Grants No. 1210378, No. ANID PIA/APOYO AFB180002, and No. Milenio-ANID-ICN2019_044. S. F. K. acknowledges the STFC Consolidated Grant No. ST/L000296/1 and the European Union's Horizon 2020 Research and Innovation programme under Marie Skłodowska-Curie grant agreement HIDDeN European ITN project (No. H2020MSCA-ITN-2019//860881-HIDDeN).

\section{APPENDIX A: ANALYTIC APPROXIMATED STEP-BY-STEP DIAGONALIZATION FOR THE CHARGED LEPTON SECTOR}

In order to diagonalize the mass matrix of Eq. (8) in an analytic way, we employ the method of mixing formalism and define intermediate mass basis. The flavor basis is used when writing the initial mass matrix of Eq. (8), whereas the true mass basis corresponds to the fully diagonalized mass matrix, which reveals the masses of all propagating charged leptons. The intermediate mass basis is a basis where the heavy particles appearing in all terms generating the entries proportional to $v_{\phi}$ are integrated out remaining other terms unrotated. This separation makes the difference between $S U(2)$ conserving and $S U(2)$ violating mixings, which will be defined later, clear, which will become important when we consider the flavor violating interactions mediated by the SM $Z$ gauge boson. Before we carry out the digonalization step-by-step, it is convenient to rearrange the mass matrix of Eq. (8) by switching the Yukawa terms by mass parameters and by swapping the fourth and fifth column in order to make the heavy vectorlike masses locate in the diagonal positions as given in Eq. (A1)

$$
M^{e}=\left(\begin{array}{c|ccccc} 
& e_{1 R} & e_{2 R} & e_{3 R} & e_{4 R} & \tilde{L}_{4 R} \\
\bar{L}_{1 L} & 0 & 0 & 0 & 0 & 0 \\
\bar{L}_{2 L} & 0 & 0 & 0 & m_{24} & 0 \\
\bar{L}_{3 L} & 0 & 0 & 0 & m_{34} & m_{35} \\
\bar{L}_{4 L} & 0 & 0 & m_{43} & 0 & M_{45}^{L} \\
\overline{\tilde{e}}_{4 L} & 0 & m_{52} & m_{53} & M_{54}^{e} & 0
\end{array}\right)=\left(\begin{array}{cccccc} 
& e_{1 R} & e_{2 R} & e_{3 R} & \tilde{L}_{4 R} & e_{4 R} \\
\bar{L}_{1 L} & 0 & 0 & 0 & 0 & 0 \\
\bar{L}_{2 L} & 0 & 0 & 0 & 0 & m_{24} \\
\bar{L}_{3 L} & 0 & 0 & 0 & m_{35} & m_{34} \\
\bar{L}_{4 L} & 0 & 0 & m_{43} & M_{45}^{L} & 0 \\
\overline{\tilde{e}}_{4 L} & 0 & m_{52} & m_{53} & 0 & M_{54}^{e}
\end{array}\right),
$$

where the indices running from 1 to 3 correspond to the three SM families, the index 4 labels the fourth vectorlike particles and lastly the index 5 denotes the tilde particles, which are a partner of the vectorlike particles. Now we are ready to diagonalize the mass matrix of Eq. (A1) and the first step is to get the intermediate mass basis and to integrate out the particles generating the entries proportional to $v_{\phi}$ (equally, all mass terms involving index 5). For this task, we first consider 34 rotation in the left-handed fields to turn off the mass term $m_{35}$.

$$
V_{34}^{L} M^{e}=\left(\begin{array}{c|ccccc} 
& e_{1 R} & e_{2 R} & e_{3 R} & \tilde{L}_{4 R} & e_{4 R} \\
\hline \bar{L}_{1 L} & 0 & 0 & 0 & 0 & 0 \\
\bar{L}_{2 L} & 0 & 0 & 0 & 0 & m_{24} \\
\bar{L}_{3 L}^{\prime} & 0 & 0 & -\frac{m_{35} m_{43}}{M_{45}^{L 1}} & 0 & \frac{m_{34} M_{45}^{L}}{M_{45}^{L L}} \\
\bar{L}_{4 L}^{\prime} & 0 & 0 & \frac{m_{43} M_{45}^{L}}{M_{45}^{L L}} & M_{45}^{L \prime} & \frac{m_{34} m_{35}^{L}}{M_{45}^{L L}} \\
\overline{\tilde{e}}_{4 L} & 0 & m_{52} & m_{53} & 0 & M_{54}^{e}
\end{array}\right)
$$




$$
M_{45}^{L \prime}=\sqrt{M_{45}^{L 2}+m_{35}^{2}}, \quad s_{34}^{L}=\frac{m_{35}}{M_{45}^{L \prime}}, \quad c_{34}^{L}=\frac{M_{45}^{L}}{M_{45}^{L \prime}}, \quad V_{34}^{L}=\left(\begin{array}{ccccc}
1 & 0 & 0 & 0 & 0 \\
0 & 1 & 0 & 0 & 0 \\
0 & 0 & c_{34}^{L} & -s_{34}^{L} & 0 \\
0 & 0 & s_{34}^{L} & c_{34}^{L} & 0 \\
0 & 0 & 0 & 0 & 1
\end{array}\right),
$$

where the primed fields correspond to the rotated fields. Throughout this whole work, the fields characterized by a capital letter are the ones belonging to a $S U(2)$ doublet under the SM gauge symmetry, whereas those ones denoted by a small letter are $S U(2)$ singlets. Then, the next rotation is 34 rotation in the right-handed leptonic fields to turn off the $m_{53}$ entry. It is worth mentioning that the order used in the rotation of the left-handed fields is 12345 , whereas for the rotation of the right-handed fields the corresponding order is 12354 since for consistency reasons we assigned the index 5 for the tilde particle,

$$
\begin{aligned}
& V_{34}^{L} M^{e}\left(V_{34}^{e}\right)^{\dagger}=\left(\begin{array}{c|ccccc} 
& e_{1 R} & e_{2 R} & e_{3 R}^{\prime} & \tilde{L}_{4 R} & e_{4 R}^{\prime} \\
\hline \bar{L}_{1 L} & 0 & 0 & 0 & 0 & 0 \\
\bar{L}_{2 L} & 0 & 0 & -\frac{m_{24} m_{53}}{M_{54}^{e \prime}} & 0 & \frac{m_{24} M_{54}^{e}}{M_{54}^{e \prime}} \\
\bar{L}_{3 L}^{\prime} & 0 & 0 & \frac{-m_{34} m_{53} M_{45}^{L} m_{35} m_{43} M_{54}^{e}}{M_{45}^{L \prime} M_{54}^{e \prime}} & 0 & \frac{-m_{35} m_{43} m_{53}+m_{34} M_{45}^{L} M_{54}^{e}}{M_{45}^{L \prime} M_{54}^{e \prime}} \\
\bar{L}_{4 L}^{\prime} & 0 & 0 & \frac{-m_{34} m_{35} m_{53}+m_{43} M_{45}^{L} M_{54}^{e}}{M_{45}^{L \prime} M_{54}^{e \prime}} & M_{45}^{L \prime} & \frac{m_{43} m_{53} M_{45}^{L}+m_{34} m_{35} M_{54}^{e}}{M_{45}^{L \prime} M_{54}^{e \prime}} \\
\overline{\tilde{e}}_{4 L} & 0 & m_{52} & 0 & 0 & M_{54}^{e \prime}
\end{array}\right), \\
& M_{54}^{e \prime}=\sqrt{M_{54}^{e 2}+m_{53}^{2}}, \quad s_{34}^{e}=\frac{m_{53}}{M_{54}^{e \prime}}, \quad c_{34}^{e}=\frac{M_{54}^{e}}{M_{54}^{e \prime}}, \quad V_{34}^{e}=\left(\begin{array}{ccccc}
1 & 0 & 0 & 0 & 0 \\
0 & 1 & 0 & 0 & 0 \\
0 & 0 & c_{34}^{e} & 0 & -s_{34}^{e} \\
0 & 0 & 0 & 1 & 0 \\
0 & 0 & s_{34}^{e} & 0 & c_{34}^{e}
\end{array}\right) \text {. }
\end{aligned}
$$

The last step to arrive at the intermediate mass basis is the 24 rotation in the right-handed fields.

$$
\begin{aligned}
& V_{34}^{L} M^{e}\left(V_{34}^{e}\right)^{\dagger}\left(V_{24}^{e}\right)^{\dagger}= \\
& \left(\begin{array}{l|ccccc} 
& e_{1 R} & e_{2 R}^{\prime} & e_{3 R}^{\prime} & \tilde{L}_{4 R} & e_{4 R}^{\prime \prime} \\
\hline \bar{L}_{1 L} & 0 & 0 & 0 & 0 & 0 \\
\bar{L}_{2 L} & 0 & -\frac{m_{24} m_{52} M_{54}^{e}}{M_{54}^{e} M_{54}^{e \prime \prime}} & -\frac{m_{24} m_{53}}{M_{54}^{e \prime}} & 0 & \frac{m_{24} M_{54}^{e}}{M_{54}^{e l \prime}} \\
\bar{L}_{3 L}^{\prime} & 0 & \frac{m_{52}\left(m_{35} m_{43} m_{53}-m_{34} M_{45}^{L} M_{54}^{e}\right)}{M_{45}^{L \prime} M_{54}^{e \prime} M_{54}^{e \prime}} & \frac{-m_{34} m_{53} M_{45}^{L}-m_{35} m_{43} M_{54}^{e}}{M_{45}^{L \prime} M_{54}^{e \prime}} & 0 & \frac{-m_{35} m_{43} m_{53}+m_{34} M_{45}^{L} M_{54}^{e}}{M_{45}^{L \prime} M_{54}^{e \prime}} \\
\bar{L}_{4 L}^{\prime} & 0 & -\frac{m_{52}\left(m_{43} M_{45}^{L} m_{53}+m_{34} m_{35} M_{54}^{e}\right)}{M_{45}^{L \prime} M_{54}^{e l} M_{54}^{e \prime \prime}} & \frac{-m_{34} m_{35} m_{53}+m_{43} M_{45}^{L} M_{54}^{e}}{M_{45}^{L \prime} M_{54}^{e \prime}} & M_{45}^{L \prime} & \frac{m_{43} m_{53} M_{45}^{L}+m_{34} m_{35} M_{54}^{e}}{M_{45}^{L \prime} M_{54}^{e \prime \prime}} \\
\overline{\tilde{e}}_{4 L} & 0 & 0 & 0 & 0 & M_{54}^{e \prime \prime}
\end{array}\right), \\
& M_{54}^{e \prime \prime}=\sqrt{M_{54}^{e \prime 2}+m_{52}^{2}}, \quad s_{24}^{e}=\frac{m_{52}}{M_{54}^{e \prime \prime}}, \quad c_{24}^{e}=\frac{M_{54}^{e \prime}}{M_{54}^{e \prime \prime}}, \quad V_{34}^{e}=\left(\begin{array}{ccccc}
1 & 0 & 0 & 0 & 0 \\
0 & c_{24}^{e} & 0 & 0 & -s_{24}^{e} \\
0 & 0 & 1 & 0 & 0 \\
0 & 0 & 0 & 1 & 0 \\
0 & s_{24}^{e} & 0 & 0 & c_{24}^{e}
\end{array}\right) .
\end{aligned}
$$


The mass matrix given in Eq. (A6) is the intermediate mass basis and this diagonalization is exactly consistent with the one for the SM charged lepton sector in one of our works [4]. When we diagonalized the charged lepton sector in [4], we assumed all off-diagonal elements to be zero and this is actually a quite suitable assumption since the differences between the Yukawa induced mass terms and the vectorlike masses are quite large. However, we consider all small mixings in order to get the fully diagonalized mass matrix in this work rather than setting them to zero, since we are interested in studying diverse FCNC constraints by scanning all possible and allowed mass ranges of the vectorlike fermions in both SM quark and lepton sectors and the FCNC constraints are sensitive to the small mixings as it will be shown below. One more feature to be mentioned in this diagonalization is that all the mixings have been made between the same $S U(2)$ multiplets. In other words, the $S U(2)$ doublet left-handed fields are mixed with the another $S U(2)$ doublet left-handed fields, whereas the $S U(2)$ singlet right-handed fields are mixed with the another $S U(2)$ singlet right-handed fields, so we call this mixing " $S U(2)$ conserving mixing." This $S U(2)$ conserving mixing can not cause the flavor violating interactions with the SM $Z$ gauge boson since they involve an identity matrix resulting from the $S M Z$ gauge interactions. Therefore, the next diagonalization process becomes especially important when we start exploring the FCNC constraints. Before we start the next diagonalization, it is convenient to reparameterize all elements of Eq. (A6)) by a simpler one as given in Eq. (A9).

$$
M^{e \prime}=V_{34}^{L} M^{e}\left(V_{34}^{e}\right)^{\dagger}\left(V_{24}^{e}\right)^{\dagger}=\left(\begin{array}{c|ccccc} 
& e_{1 R} & e_{2 R}^{\prime} & e_{3 R}^{\prime} & \tilde{L}_{4 R} & e_{4 R}^{\prime \prime} \\
\hline \bar{L}_{1 L} & 0 & 0 & 0 & 0 & 0 \\
\bar{L}_{2 L} & 0 & m_{22}^{\prime} & m_{23}^{\prime} & 0 & m_{24}^{\prime} \\
\bar{L}_{3 L}^{\prime} & 0 & m_{32}^{\prime} & m_{33}^{\prime} & 0 & m_{34}^{\prime} \\
\bar{L}_{4 L}^{\prime} & 0 & m_{42}^{\prime} & m_{43}^{\prime} & M_{45}^{L \prime} & m_{44}^{\prime} \\
\tilde{e}_{4 L} & 0 & 0 & 0 & 0 & M_{54}^{e \prime \prime}
\end{array}\right)
$$

We carry out first the 35 rotation in the left-handed fields of mass matrix (A9) and this is a start of "SU(2) violating mixing." As already mentioned, since the difference between the Yukawa mass and vectorlike mass is significantly sizeable, it is possible to simplify the mixing matrices in terms of the relevant small mixing angle $\theta_{35}^{L}$.

$V_{35}^{L} M^{e \prime}=\left(\begin{array}{c|ccccc} & e_{1 R} & e_{2 R}^{\prime} & e_{3 R}^{\prime} & \tilde{L}_{4 R} & e_{4 R}^{\prime \prime} \\ \hline \bar{L}_{1 L} & 0 & 0 & 0 & 0 & 0 \\ \bar{L}_{2 L} & 0 & m_{22}^{\prime} & m_{23}^{\prime} & 0 & m_{24}^{\prime} \\ \bar{L}_{3 L}^{\prime \prime} & 0 & m_{32}^{\prime} & m_{33}^{\prime} & 0 & m_{34}^{\prime}-M_{54}^{e \prime} \theta_{35}^{L} \\ \bar{L}_{4 L}^{\prime} & 0 & m_{42}^{\prime} & m_{43}^{\prime} & M_{45}^{L \prime} & m_{44}^{\prime} \\ \bar{e}_{4 L}^{\prime} & 0 & m_{32}^{\prime} \theta_{35}^{L} & m_{33}^{\prime} \theta_{35}^{L} & 0 & M_{54}^{e \prime \prime}+m_{34}^{\prime} \theta_{35}^{L}\end{array}\right) \approx\left(\begin{array}{cccccc} & e_{1 R} & e_{2 R}^{\prime} & e_{3 R}^{\prime} & \tilde{L}_{4 R} & e_{4 R}^{\prime \prime} \\ \bar{L}_{1 L} & 0 & 0 & 0 & 0 & 0 \\ \bar{L}_{2 L} & 0 & m_{22}^{\prime} & m_{23}^{\prime} & 0 & m_{24}^{\prime} \\ \bar{L}_{3 L}^{\prime \prime} & 0 & m_{32}^{\prime} & m_{33}^{\prime} & 0 & 0 \\ \bar{L}_{4 L}^{\prime} & 0 & m_{42}^{\prime} & m_{43}^{\prime} & M_{45}^{L \prime} & m_{44}^{\prime} \\ \tilde{\tilde{e}}_{4 L}^{\prime} & 0 & 0 & 0 & 0 & M_{54}^{e \prime \prime}\end{array}\right)$

$$
\theta_{35}^{L}=\frac{m_{34}^{\prime}}{M_{54}^{e \prime}}, \quad V_{35}^{L}=\left(\begin{array}{ccccc}
1 & 0 & 0 & 0 & 0 \\
0 & 1 & 0 & 0 & 0 \\
0 & 0 & 1 & 0 & -\theta_{35}^{L} \\
0 & 0 & 0 & 1 & 0 \\
0 & 0 & \theta_{35}^{L} & 0 & 1
\end{array}\right)
$$

The next step is the 25 rotation in the left-handed fields to turn off the mass term $m_{24}^{\prime}$. 
$V_{25}^{L} V_{35}^{L} M^{e \prime}=\left(\begin{array}{c|ccccc} & e_{1 R} & e_{2 R}^{\prime} & e_{3 R}^{\prime} & \tilde{L}_{4 R} & e_{4 R}^{\prime \prime} \\ \hline \bar{L}_{1 L} & 0 & 0 & 0 & 0 & 0 \\ \bar{L}_{2 L}^{\prime} & 0 & m_{22}^{\prime} & m_{23}^{\prime} & 0 & m_{24}^{\prime}-M_{54}^{e \prime \prime} \theta_{25}^{L} \\ \bar{L}_{3 L}^{\prime \prime} & 0 & m_{32}^{\prime} & m_{33}^{\prime} & 0 & 0 \\ \bar{L}_{4 L}^{\prime} & 0 & m_{42}^{\prime} & m_{43}^{\prime} & M_{45}^{L \prime} & m_{44}^{\prime} \\ \overline{\tilde{e}}_{4 L}^{\prime \prime} & 0 & m_{22}^{\prime} \theta_{25}^{L} & m_{23}^{\prime} \theta_{25}^{L} & 0 & M_{54}^{e \prime \prime}+m_{24}^{\prime} \theta_{25}^{L}\end{array}\right) \approx\left(\begin{array}{cccccc} & e_{1 R} & e_{2 R}^{\prime} & e_{3 R}^{\prime} & \tilde{L}_{4 R} & e_{4 R}^{\prime \prime} \\ \bar{L}_{1 L} & 0 & 0 & 0 & 0 & 0 \\ \bar{L}_{2 L}^{\prime} & 0 & m_{22}^{\prime} & m_{23}^{\prime} & 0 & 0 \\ \bar{L}_{3 L}^{\prime \prime} & 0 & m_{32}^{\prime} & m_{33}^{\prime} & 0 & 0 \\ \bar{L}_{4 L}^{\prime} & 0 & m_{42}^{\prime} & m_{43}^{\prime} & M_{45}^{L \prime} & m_{44}^{\prime} \\ \overline{\tilde{e}}_{4 L}^{\prime \prime} & 0 & 0 & 0 & 0 & M_{54}^{e \prime \prime}\end{array}\right)$

$$
\theta_{25}^{L}=\frac{m_{24}^{\prime}}{M_{54}^{e \prime \prime}}, \quad V_{25}^{L}=\left(\begin{array}{ccccc}
1 & 0 & 0 & 0 & 0 \\
0 & 1 & 0 & 0 & -\theta_{25}^{L} \\
0 & 0 & 1 & 0 & 0 \\
0 & 0 & 0 & 1 & 0 \\
0 & \theta_{25}^{L} & 0 & 0 & 1
\end{array}\right)
$$

The next is the 35 rotation in the right-handed fields to turn off the mass term $m_{42}^{\prime}$.

$$
\begin{gathered}
V_{25}^{L} V_{35}^{L} M^{e \prime}\left(V_{35}^{e}\right)^{\dagger}=\left(\begin{array}{c|ccccc} 
& e_{1 R} & e_{2 R}^{\prime} & e_{3 R}^{\prime \prime} & \tilde{L}_{4 R}^{\prime} & e_{4 R}^{\prime \prime} \\
\hline \bar{L}_{1 L} & 0 & 0 & 0 & 0 & 0 \\
\bar{L}_{2 L}^{\prime} & 0 & m_{22}^{\prime} & m_{23}^{\prime} & m_{23}^{\prime} \theta_{35}^{e} & 0 \\
\bar{L}_{3 L}^{\prime \prime} & 0 & m_{32}^{\prime} & m_{33}^{\prime} & m_{33}^{\prime} \theta_{35}^{e} & 0 \\
\bar{L}_{4 L}^{\prime} & 0 & m_{42}^{\prime} & m_{43}^{\prime}-M_{45}^{L \prime} \theta_{35}^{e} & M_{45}^{L \prime}+m_{43}^{\prime} \theta_{35}^{e} & m_{44}^{\prime} \\
\bar{e}_{4 L}^{\prime \prime} & 0 & 0 & 0 & 0 & M_{54}^{e \prime \prime}
\end{array}\right) \approx\left(\begin{array}{ccccccc} 
& e_{1 R} & e_{2 R}^{\prime} & e_{3 R}^{\prime \prime} & \tilde{L}_{4 R}^{\prime} & e_{4 R}^{\prime \prime} \\
\hline \bar{L}_{1 L} & 0 & 0 & 0 & 0 & 0 \\
\bar{L}_{2 L}^{\prime} & 0 & m_{22}^{\prime} & m_{23}^{\prime} & 0 & 0 \\
\bar{L}_{3 L}^{\prime \prime} & 0 & m_{32}^{\prime} & m_{33}^{\prime} & 0 & 0 \\
\bar{L}_{4 L}^{\prime} & 0 & m_{42}^{\prime} & 0 & M_{45}^{L \prime} & m_{44}^{\prime} \\
\bar{e}_{4 L}^{\prime \prime} & 0 & 0 & 0 & 0 & M_{54}^{e \prime \prime}
\end{array}\right) \\
\theta_{35}^{e}=\frac{m_{43}^{\prime}}{M_{45}^{L \prime},} V_{35}^{e}=\left(\begin{array}{ccccc}
1 & 0 & 0 & 0 & 0 \\
0 & 1 & 0 & 0 & 0 \\
0 & 0 & 1 & -\theta_{35}^{e} & 0 \\
0 & 0 & \theta_{35}^{e} & 1 & 0 \\
0 & 0 & 0 & 0 & 1
\end{array}\right) \\
\end{gathered}
$$

After performing the right-handed 25 rotation, we have a mass matrix, whose form is block diagonal.

$V_{25}^{L} V_{35}^{L} M^{e \prime}\left(V_{35}^{e}\right)^{\dagger}\left(V_{25}^{e}\right)^{\dagger}=$

$$
\left(\begin{array}{c|ccccc} 
& e_{1 R} & e_{2 R}^{\prime \prime} & e_{3 R}^{\prime \prime} & \tilde{L}_{4 R}^{\prime \prime} & e_{4 R}^{\prime \prime} \\
\hline \bar{L}_{1 L} & 0 & 0 & 0 & 0 & 0 \\
\bar{L}_{2 L}^{\prime} & 0 & m_{22}^{\prime} & m_{23}^{\prime} & m_{22}^{\prime} \theta_{25}^{e} & 0 \\
\bar{L}_{3 L}^{\prime \prime} & 0 & m_{32}^{\prime} & m_{33}^{\prime} & m_{32}^{\prime} \theta_{25}^{e} & 0 \\
\bar{L}_{4 L}^{\prime \prime} & 0 & m_{42}^{\prime}-M_{45}^{L \prime} & 0 & M_{45}^{L \prime}+m_{42}^{\prime} & m_{44}^{\prime} \\
\overline{\tilde{e}}_{4 L}^{\prime \prime} & 0 & 0 & 0 & 0 & M_{54}^{\prime \prime}
\end{array}\right) \approx\left(\begin{array}{ccccccc} 
& e_{1 R} & e_{2 R}^{\prime \prime} & e_{3 R}^{\prime \prime} & \tilde{L}_{4 R}^{\prime \prime} & e_{4 R}^{\prime \prime} \\
\bar{L}_{1 L} & 0 & 0 & 0 & 0 & 0 \\
\bar{L}_{2 L}^{\prime} & 0 & m_{22}^{\prime} & m_{23}^{\prime} & 0 & 0 \\
\bar{L}_{3 L}^{\prime \prime} & 0 & m_{32}^{\prime} & m_{33}^{\prime} & 0 & 0 \\
\bar{L}_{4 L}^{\prime} & 0 & 0 & 0 & M_{45}^{L \prime} & m_{44}^{\prime} \\
\overline{\tilde{e}}_{4 L}^{\prime \prime} & 0 & 0 & 0 & 0 & M_{54}^{e \prime \prime}
\end{array}\right)
$$




$$
\theta_{25}^{e}=\frac{m_{42}^{\prime}}{M_{45}^{L \prime}}, \quad V_{25}^{e}=\left(\begin{array}{ccccc}
1 & 0 & 0 & 0 & 0 \\
0 & 1 & 0 & -\theta_{25}^{e} & 0 \\
0 & 0 & 1 & 0 & 0 \\
0 & \theta_{25}^{e} & 0 & 1 & 0 \\
0 & 0 & 0 & 0 & 1
\end{array}\right)
$$

We arrive at the fully diagonalized mass matrix by diagonalizing the upper-left $3 \times 3$ block as well as the lower-right $2 \times 2$ block as shown below in Eq. (A19).

$$
\begin{array}{r}
V_{45}^{L} V_{23}^{L} V_{35}^{L} V_{25}^{L} M^{e \prime}\left(V_{35}^{e}\right)^{\dagger}\left(V_{25}^{e}\right)^{\dagger}\left(V_{23}^{e}\right)^{\dagger}\left(V_{54}^{e}\right)^{\dagger}=\operatorname{diag}\left(0, m_{\mu}, m_{\tau}, M_{E_{4}}, M_{\tilde{E}_{4}}\right) \\
V_{45}^{L} V_{23}^{L} V_{35}^{L} V_{25}^{L} V_{34}^{L} M^{e}\left(V_{34}^{e}\right)^{\dagger}\left(V_{24}^{e}\right)^{\dagger}\left(V_{35}^{e}\right)^{\dagger}\left(V_{25}^{e}\right)^{\dagger}\left(V_{23}^{e}\right)^{\dagger}\left(V_{54}^{e}\right)^{\dagger}=\operatorname{diag}\left(0, m_{\mu}, m_{\tau}, M_{E_{4}}, M_{\tilde{E}_{4}}\right)
\end{array}
$$

As mentioned in the Introduction, the SM charged lepton belonging to the first family, namely, the electron does not acquire a mass with one vectorlike family as seen in Eq. (A19). This is due to the fact that the model under consideration has two leptonic seesaw mediators, which provide tree-level masses to the muon and tau leptons. It is worth mentioning that the number of seesaw mediators has to be larger or equal than the number of SM fermion families in order to provide masses to the SM fermions. A nonvanishing electron mass can be generated by introducing one extra vectorlike family as done in the Ref. [4]. Then, we can easily confirm how the SM charged leptons in the flavor basis are connected with those ones in the mass basis via the following unitary mixing matrices.

$$
\begin{aligned}
& \left(\begin{array}{c}
e_{L} \\
\mu_{L} \\
\tau_{L} \\
E_{4 L} \\
\tilde{E}_{4 L}
\end{array}\right)=\left(\begin{array}{c}
e_{1 L} \\
e_{2 L}^{\prime} \\
e_{3 L}^{\prime \prime} \\
e_{4 L}^{\prime} \\
\tilde{e}_{4 L}^{\prime \prime}
\end{array}\right)=V^{L}\left(\begin{array}{c}
e_{1 L} \\
e_{2 L} \\
e_{3 L} \\
e_{4 L} \\
\tilde{e}_{4 L}
\end{array}\right)=V_{45}^{L} V_{23}^{L} V_{25}^{L} V_{35}^{L} V_{34}^{L}\left(\begin{array}{c}
e_{1 L} \\
e_{2 L} \\
e_{3 L} \\
e_{4 L} \\
\tilde{e}_{4 L}
\end{array}\right), \\
& \left(\begin{array}{c}
e_{R} \\
\mu_{R} \\
\tau_{R} \\
\tilde{E}_{4 R} \\
E_{4 R}
\end{array}\right)=\left(\begin{array}{c}
e_{1 R} \\
e_{2 R}^{\prime \prime} \\
e_{3 R}^{\prime \prime} \\
\tilde{e}_{4 R}^{\prime \prime} \\
e_{4 R}^{\prime \prime}
\end{array}\right)=V^{e}\left(\begin{array}{c}
e_{1 R} \\
e_{2 R} \\
e_{3 R} \\
\tilde{e}_{4 R} \\
e_{4 R}
\end{array}\right)=V_{54}^{e} V_{23}^{e} V_{25}^{e} V_{35}^{e} V_{24}^{e} V_{34}^{e}\left(\begin{array}{c}
e_{1 R} \\
e_{2 R} \\
e_{3 R} \\
\tilde{e}_{4 R} \\
e_{4 R}
\end{array}\right) .
\end{aligned}
$$

The left-handed 34 mixing $V_{34}^{L}$ and right-handed 24,34 mixings $V_{24,34}^{e}$ are the $S U(2)$ conserving mixings, whereas the left-handed 25,35 mixings $V_{25,35}^{L}$ and right-handed 25,35 mixings $V_{25,35}^{e}$ are the $S U(2)$ violating mixings. We will see that these $S U(2)$ violating mixings play a crucial role in generating the renormalizable flavor violating mixings mediated by the SM $Z$ gauge boson in Sec. IV. It is worth mentioning that this step-by-step diagonalization is a quite good approximation to the corresponding numerical diagonalization carried out by the singular value decomposition (SVD) method since the former yields similar results to the ones obtained from the latter, with very small differences due to the fact that all off-diagonal elements resulting from the step-by-step diagonalization are quite negligible and thus they can be approximated to zero, as discussed in detail in Appendix D.

\section{APPENDIX B: ANALYTIC APPROXIMATED STEP-BY-STEP DIAGONALIZATION FOR THE UP-QUARK SECTOR}

The initial mass matrix for the up-type quark sector in the flavor basis is given by: 


$$
M^{u}=\left(\begin{array}{c|ccccc} 
& u_{1 R} & u_{2 R} & u_{3 R} & u_{4 R} & \tilde{Q}_{4 R} \\
\hline \bar{Q}_{1 L} & 0 & 0 & 0 & 0 & 0 \\
\bar{Q}_{2 L} & 0 & 0 & 0 & y_{24}^{u} v_{u} & 0 \\
\bar{Q}_{3 L} & 0 & 0 & 0 & y_{34}^{u} v_{u} & x_{34}^{Q} v_{\phi} \\
\bar{Q}_{4 L} & 0 & 0 & y_{43}^{u} v_{u} & 0 & M_{44}^{Q} \\
\overline{\tilde{u}}_{4 L} & 0 & x_{42}^{u} v_{\phi} & x_{43}^{u} v_{\phi} & M_{44}^{u} & 0
\end{array}\right)=\left(\begin{array}{cccccc} 
& u_{1 R} & u_{2 R} & u_{3 R} & \tilde{Q}_{4 R} & u_{4 R} \\
\hline \bar{Q}_{1 L} & 0 & 0 & 0 & 0 & 0 \\
\bar{Q}_{2 L} & 0 & 0 & 0 & 0 & m_{24}^{u} \\
\bar{Q}_{3 L} & 0 & 0 & 0 & m_{35}^{u} & m_{34}^{u} \\
\bar{Q}_{4 L} & 0 & 0 & m_{43}^{u} & M_{44}^{Q} & 0 \\
\overline{\tilde{u}}_{4 L} & 0 & m_{52}^{u} & m_{53}^{u} & 0 & M_{44}^{u}
\end{array}\right),
$$

The mass matrix of Eq. (B1) in the flavor basis is exactly consistent with the one corresponding to the charged lepton sector excepting for a few substitutions $y^{e} \rightarrow y^{u}, v_{d} \rightarrow v_{u}, x^{L} \rightarrow x^{Q}$ and $x^{e} \rightarrow x^{u}$. However, these substitutions do not change the whole structure of the mass matrix, so we do not need to derive all the required mixings from the initial mass matrix, instead the given mixings in the charged lepton sector can be reused as follows (For the charged lepton sector, it is enough to notice the symbol $L$ means left-handed doublet and $e$ means right-handed singlet. However, it becomes complicated in the quark sector since the mass matrices in the up- and down-type quark have a different form, so we change the mixing notation by $V_{L(R)}^{u, d}$ instead of $V^{Q}$.):

$$
\begin{aligned}
& \left(\begin{array}{c}
u_{L} \\
c_{L} \\
t_{L} \\
U_{4 L} \\
\tilde{U}_{4 L}
\end{array}\right)=\left(\begin{array}{c}
u_{1 L} \\
u_{2 L}^{\prime} \\
u_{3 L}^{\prime \prime} \\
u_{4 L}^{\prime \prime} \\
\tilde{u}_{4 L}^{\prime \prime}
\end{array}\right)=V_{L}^{u}\left(\begin{array}{c}
u_{1 L} \\
u_{2 L} \\
u_{3 L} \\
u_{4 L} \\
\tilde{u}_{4 L}
\end{array}\right)=\left(V_{L}^{u}\right)_{45}\left(V_{L}^{u}\right)_{23}\left(V_{L}^{u}\right)_{25}\left(V_{L}^{u}\right)_{35}\left(V_{L}^{u}\right)_{34}\left(\begin{array}{c}
u_{1 L} \\
u_{2 L} \\
u_{3 L} \\
u_{4 L} \\
\tilde{u}_{4 L}
\end{array}\right), \\
& \left(\begin{array}{c}
u_{R} \\
c_{R} \\
t_{R} \\
\tilde{U}_{4 R} \\
U_{4 R}
\end{array}\right)=\left(\begin{array}{c}
u_{1 R} \\
u_{2 R}^{\prime \prime} \\
u_{3 R}^{\prime \prime} \\
\tilde{u}_{4 R}^{\prime \prime} \\
u_{4 R}^{\prime \prime}
\end{array}\right)=V_{R}^{u}\left(\begin{array}{c}
u_{1 R} \\
u_{2 R} \\
u_{3 R} \\
\tilde{u}_{4 R} \\
u_{4 R}
\end{array}\right)=\left(V_{R}^{u}\right)_{54}\left(V_{R}^{u}\right)_{23}\left(V_{R}^{u}\right)_{25}\left(V_{R}^{u}\right)_{35}\left(V_{R}^{u}\right)_{24}\left(V_{R}^{u}\right)_{34}\left(\begin{array}{c}
u_{1 R} \\
u_{2 R} \\
u_{3 R} \\
\tilde{u}_{4 R} \\
u_{4 R}
\end{array}\right) .
\end{aligned}
$$

As mentioned in Sec. III B, this approximated step-by-step diagonalization for the up-quark sector requires more caution since some of the off-diagonal elements being of order unity and appearing as a result of mixings can be sizeable due to the heavy top quark mass and the heavy exotic up type quark masses thus requiring the use of the numerical SVD technique for the correct diagonalization and the SVD diagonalization will be used in our numerical scans in the main body of this work. The comparison between a numerical mixing matrix derived from the SVD method and the one obtained from the analytic perturbative diagonalization will be discussed in Appendix E.

\section{APPENDIX C: ANALYTIC APPROXIMATED STEP-BY-STEP DIAGONALIZATION FOR THE DOWN-QUARK SECTOR}

We start from the initial down-type mass matrix given in Eq. (14) in the flavor basis.

$$
M^{d}=\left(\begin{array}{c|ccccc} 
& d_{1 R} & d_{2 R} & d_{3 R} & d_{4 R} & \tilde{Q}_{4 R} \\
\hline \bar{Q}_{1 L} & 0 & 0 & 0 & y_{14}^{d} v_{d} & 0 \\
\bar{Q}_{2 L} & 0 & 0 & 0 & y_{24}^{d} v_{d} & 0 \\
\bar{Q}_{3 L} & 0 & 0 & 0 & y_{34}^{d} v_{d} & x_{34}^{Q} v_{\phi} \\
\bar{Q}_{4 L} & 0 & 0 & y_{43}^{d} v_{d} & 0 & M_{44}^{Q} \\
\overline{\tilde{d}}_{4 L} & 0 & x_{42}^{d} v_{\phi} & x_{43}^{d} v_{\phi} & M_{44}^{d} & 0
\end{array}\right)
$$

As in the charged lepton case, it is convenient to rearrange the Yukawa mass terms by mass parameters and to swap the fourth and fifth column. 


$$
M^{d}=\left(\begin{array}{c|ccccc} 
& d_{1 R} & d_{2 R} & d_{3 R} & d_{4 R} & \tilde{Q}_{4 R} \\
\bar{Q}_{1 L} & 0 & 0 & 0 & m_{14}^{d} & 0 \\
\bar{Q}_{2 L} & 0 & 0 & 0 & m_{24}^{d} & 0 \\
\bar{Q}_{3 L} & 0 & 0 & 0 & m_{34}^{d} & m_{35}^{d} \\
\bar{Q}_{4 L} & 0 & 0 & m_{43}^{d} & 0 & M_{44}^{Q} \\
\overline{\tilde{d}}_{4 L} & 0 & m_{52}^{d} & m_{53}^{d} & M_{44}^{d} & 0
\end{array}\right)=\left(\begin{array}{c|ccccc} 
& d_{1 R} & d_{2 R} & d_{3 R} & \tilde{Q}_{4 R} & d_{4 R} \\
\bar{Q}_{1 L} & 0 & 0 & 0 & 0 & m_{14}^{d} \\
\bar{Q}_{2 L} & 0 & 0 & 0 & 0 & m_{24}^{d} \\
\bar{Q}_{3 L} & 0 & 0 & 0 & m_{35}^{d} & m_{34}^{d} \\
\bar{Q}_{4 L} & 0 & 0 & m_{43}^{d} & M_{44}^{Q} & 0 \\
\overline{\tilde{d}}_{4 L} & 0 & m_{52}^{d} & m_{53}^{d} & 0 & M_{44}^{d}
\end{array}\right)
$$

In order to proceed from the flavor basis to the intermediate mass basis, the first thing to do is to carry out the $S U(2)$ conserving mixings $\theta_{34 L}^{d}$ and $\theta_{24,34 R}^{d}$ and we display the intermediate mass matrix for the down-type quarks without middle steps since the process is exactly same as the charged lepton case (After calculating all mixings required, we simplified the calculated mass parameters by $m^{\prime}$ ).

$$
M^{d \prime}=V_{34 L}^{d} M^{d}\left(V_{34 R}^{d}\right)^{\dagger}\left(V_{24 R}^{d}\right)^{\dagger}=\left(\begin{array}{c|ccccc} 
& d_{1 R} & d_{2 R}^{\prime} & d_{3 R}^{\prime} & \tilde{d}_{4 R} & d_{4 R}^{\prime \prime} \\
\hline \bar{d}_{1 L} & 0 & m_{12}^{d \prime} & m_{13}^{d \prime} & 0 & m_{14}^{d \prime} \\
\bar{d}_{2 L} & 0 & m_{22}^{d \prime} & m_{23}^{d \prime} & 0 & m_{24}^{d \prime} \\
\bar{d}_{3 L}^{d \prime} & 0 & m_{32}^{d \prime} & m_{33}^{d \prime} & 0 & m_{34}^{d \prime} \\
\bar{d}_{4 L}^{\prime \prime} & 0 & m_{42}^{d \prime} & m_{43}^{d \prime} & M_{45}^{Q \prime} & m_{44}^{d \prime} \\
\overline{\tilde{d}}_{4 L} & 0 & 0 & 0 & 0 & M_{54}^{d \prime \prime}
\end{array}\right)
$$

We should carry out the $S U(2)$ violating mixings to turn off the mass parameters $m_{14,24,34,42,43}^{d \prime}$ and the mixing angles are very suppressed by the ratio between Yukawa and vectorlike masses. Then the block diagonal form of this mass matrix appears as follows:

$$
M^{d \prime \prime}=V_{15 L}^{d} V_{25 L}^{d} V_{35 L}^{d} M^{d \prime}\left(V_{35 R}^{d}\right)^{\dagger}\left(V_{25 R}^{d}\right)^{\dagger}=\left(\begin{array}{c|ccccc} 
& d_{1 R} & d_{2 R}^{\prime \prime} & d_{3 R}^{\prime \prime} & \tilde{d}_{4 R}^{\prime \prime} & d_{4 R}^{\prime \prime} \\
\hline \bar{d}_{1 L}^{\prime} & 0 & m_{12}^{d \prime} & m_{13}^{d \prime} & 0 & 0 \\
\bar{d}_{2 L}^{\prime \prime} & 0 & m_{22}^{d \prime} & m_{23}^{d \prime} & 0 & 0 \\
\bar{d}_{3 L}^{\prime \prime} & 0 & m_{32}^{d \prime} & m_{33}^{d \prime} & 0 & 0 \\
\bar{d}_{4 L}^{\prime \prime} & 0 & 0 & 0 & M_{45}^{Q \prime} & m_{44}^{d \prime} \\
\overline{\tilde{d}}_{4 L}^{\prime \prime \prime} & 0 & 0 & 0 & 0 & M_{54}^{d \prime \prime}
\end{array}\right)
$$

An important feature of the mass matrix of Eq. (C4) is the mass parameters of the first row is proportional to those of the second row by a factor (In other words, $m_{12}^{d \prime} / m_{22}^{d \prime}=m_{13}^{d \prime} / m_{23}^{d \prime}$. We follow the convention to diagonalize the upper-left $3 \times 3$ block [28] rather than simply rotating the upper-left block. As the mass matrix of Eq. (C4) consists of only real numbers, we can exclude the complex numbers in the convention and the convention is given by:

$$
V_{12 L}^{d} V_{13 L}^{d} V_{23 L}^{d} M^{d \prime \prime}\left(V_{23 R}^{d}\right)^{\dagger}\left(V_{13 R}^{d}\right)^{\dagger}\left(V_{12 R}^{d}\right)^{\dagger}=\operatorname{diag}\left(0, m_{s}, m_{b}, M_{D_{4}}, M_{\tilde{D}_{4}}\right)
$$

and then we arrive to the fully diagonalized mass matrix, which reveals all propagating mass for the down-type quarks. Then, the connection from the flavor to mass basis for the down-type quarks can be seen via the unitary mixing matrices as follows (notice that the right-handed down-type quark mixing matrices $\left(V_{R}^{d}\right)_{12,13}$ remain as an identity matrix as the relevant mass matrix has the form of $\left(\begin{array}{cc}0 & m_{a} \\ 0 & m_{b}\end{array}\right)$ and this form generally induces only left-handed mixing matrices). 


$$
\begin{aligned}
& \left(\begin{array}{c}
d_{L} \\
s_{L} \\
b_{L} \\
D_{4 L} \\
\tilde{D}_{4 L}
\end{array}\right)=\left(\begin{array}{l}
d_{1 L}^{\prime \prime \prime} \\
d_{2 L}^{\prime \prime \prime} \\
d_{3 L}^{\prime \prime \prime} \\
d_{4 L}^{\prime \prime \prime} \\
\tilde{d}_{4 L}^{\prime \prime \prime}
\end{array}\right)=V_{L}^{d}\left(\begin{array}{c}
d_{1 L} \\
d_{2 L} \\
d_{3 L} \\
d_{4 L} \\
\tilde{d}_{4 L}
\end{array}\right)=\left(V_{L}^{d}\right)_{45}\left(V_{L}^{d}\right)_{12}\left(V_{L}^{d}\right)_{13}\left(V_{L}^{d}\right)_{23}\left(V_{L}^{d}\right)_{15}\left(V_{L}^{d}\right)_{25}\left(V_{L}^{d}\right)_{35}\left(V_{L}^{d}\right)_{34}\left(\begin{array}{c}
d_{1 L} \\
d_{2 L} \\
d_{3 L} \\
d_{4 L} \\
\tilde{d}_{4 L}
\end{array}\right) \\
& \left(\begin{array}{c}
d_{R} \\
s_{R} \\
b_{R} \\
D_{4 R} \\
\tilde{D}_{4 R}
\end{array}\right)=\left(\begin{array}{l}
d_{1 R} \\
d_{2 R}^{\prime \prime \prime} \\
d_{3 R}^{\prime \prime \prime} \\
\tilde{d}_{4 R}^{\prime \prime \prime} \\
d_{4 R}^{\prime \prime \prime}
\end{array}\right)=V_{R}^{d}\left(\begin{array}{c}
d_{1 R} \\
d_{2 R} \\
d_{3 R} \\
\tilde{d}_{4 R} \\
d_{4 R}
\end{array}\right)=\left(V_{R}^{d}\right)_{54}\left(V_{R}^{d}\right)_{23}\left(V_{R}^{d}\right)_{25}\left(V_{R}^{d}\right)_{35}\left(V_{R}^{d}\right)_{24}\left(V_{R}^{d}\right)_{34}\left(\begin{array}{c}
d_{1 R} \\
d_{2 R} \\
d_{3 R} \\
\tilde{d}_{4 R} \\
d_{4 R}
\end{array}\right)
\end{aligned}
$$

\section{APPENDIX D: NUMERICAL COMPARISON FOR THE CHARGED LEPTON SECTOR}

We have previously stressed that the analytical charged lepton mixing matrix is quite close to the numerical one and we will compare them in this Appendix. For this comparison, we start from the charged lepton mass matrix in the flavor basis, evaluated in one of the benchmark points used in our numerical scans:

$$
M^{e}=\left(\begin{array}{ccccc}
0 & 0 & 0 & 0 & 0 \\
0 & 0 & 0 & 0 & -2.151 \\
0 & 0 & 0 & 161.657 & 3.955 \\
0 & 0 & 4.600 & 536.050 & 0 \\
0 & 51.135 & 97.915 & 0 & 696.178
\end{array}\right)
$$

Firstly, we evaluate the mixing matrices $V^{L, e}$ using the analytic mixings of Eq. (A20). The analytic mixing matrices $V_{\text {ana }}^{L, e}$ are given by:

$$
\begin{aligned}
V_{\text {ana }}^{L}= & \left(\begin{array}{ccccc}
1 . & 0 . & 0 . & 0 . & 0 . \\
0 . & 0.985598 & 0.161888 & -0.0488209 & 0.00211728 \\
0 . & 0.169076 & -0.943613 & 0.284567 & 0.00548067 \\
0 . & 0.00002015 & 0.288689 & 0.957399 & -0.00668649 \\
0 . & -0.00301343 & 0.00675946 & 0.00494558 & 0.99996
\end{array}\right) \\
V_{\text {ana }}^{e}= & \left(\begin{array}{ccccc}
1 . & 0 . & 0 . & 0 . & 0 . \\
0 . & 0.986254 & -0.157386 & 0.00134068 & -0.050305 \\
0 . & 0.14846 & 0.97769 & -0.00738828 & -0.148413 \\
0 . & -0.000610678 & 0.00669679 & 0.999958 & -0.00627468 \\
0 . & 0.0725407 & 0.138946 & 0.00531106 & 0.987625
\end{array}\right) .
\end{aligned}
$$

Notice that the mixing matrices $V_{L, R}^{u}$ and $V_{R}^{d}$ have exactly the same structure than the charged lepton mixing matrix since all off-diagonal elements in the first row and column are zero, however the mixing matrix $V_{L}^{d}$ is different as it can have mixings with the down-type first generation as seen in Eq. (15). 
The mixing matrices $V_{\text {num }}^{L, e}$ derived by the numerical SVD are given by:

$$
\begin{aligned}
V_{\text {num }}^{L}= & \left(\begin{array}{ccccc}
1 . & 0 . & 0 . & 0 . & 0 . \\
0 . & 0.985598 & 0.161888 & -0.0488206 & 0.00211728 \\
0 . & 0.169076 & -0.94362 & 0.284543 & 0.00548077 \\
0 . & -0.0000241021 & -0.288666 & -0.957407 & 0.00668612 \\
0 . & -0.00301342 & 0.00675933 & 0.00494538 & 0.99996
\end{array}\right) \\
V_{\text {num }}^{e}= & \left(\begin{array}{ccccc}
1 . & 0 . & 0 . & 0 . & 0 . \\
0 . & 0.986254 & -0.157386 & 0.00134068 & -0.0503047 \\
0 . & 0.148461 & 0.977693 & -0.00738841 & -0.148398 \\
0 . & 0.000610624 & -0.00669703 & -0.999958 & 0.00627442 \\
0 . & 0.0725382 & 0.138932 & 0.00531088 & 0.987627
\end{array}\right)
\end{aligned}
$$

The difference between the mixing matrices can be easily seen by subtracting one from another after taking absolute value.

$$
\begin{aligned}
\left|V_{\text {ana }}^{L}\right|-\left|V_{\text {num }}^{L}\right| & =\left(\begin{array}{ccccc}
0 & 0 & 0 & 0 & 0 \\
0 & 0 & -7.58945 \times 10^{-8} & 2.51664 \times 10^{-7} & 5.97371 \times 10^{-10} \\
0 & 3.46904 \times 10^{-10} & -7.19169 \times 10^{-6} & 2.38502 \times 10^{-5} & -1.00783 \times 10^{-7} \\
0 & -3.95207 \times 10^{-6} & 2.35474 \times 10^{-5} & -7.10253 \times 10^{-6} & 3.68376 \times 10^{-7} \\
0 & 9.97344 \times 10^{-9} & 1.29353 \times 10^{-7} & 2.03736 \times 10^{-7} & -1.91205 \times 10^{-9}
\end{array}\right) \\
\left|V_{\text {ana }}^{e}\right|-\left|V_{\text {num }}^{e}\right|= & \left(\begin{array}{ccccc}
0 & 0 & 0 & 0 & 0 \\
0 & -2.39211 \times 10^{-8} & 4.58026 \times 10^{-8} & 9.72974 \times 10^{-10} & 3.25661 \times 10^{-7} \\
0 & -1.06479 \times 10^{-6} & -2.03783 \times 10^{-6} & -1.29588 \times 10^{-7} & 1.44968 \times 10^{-5} \\
0 & 5.47244 \times 10^{-8} & -2.43141 \times 10^{-7} & 0 & 2.58046 \times 10^{-7} \\
0 & 2.50398 \times 10^{-6} & 1.42997 \times 10^{-5} & 1.84594 \times 10^{-7} & -2.19659 \times 10^{-6}
\end{array}\right)
\end{aligned}
$$

Therefore we have confirmed that the analytic mixing matrix for the charged lepton sector is quite close to one obtained from the numerical SVD diagonalization. Using the numerical mixing matrices derived by the SVD diagonalization, we confirm the following $D_{L, R}^{e \prime}$ matrices of $Z$ couplings with leptons of Eq. (21) as follows (we included here the prefactor $\left.g / c_{w}\right)$ :

$$
\begin{gathered}
D_{L}^{e \prime}=\left(\begin{array}{ccccc}
-2.01645 \times 10^{-1} & 0 . & 0 . & 0 . & 0 . \\
0 . & -2.01643 \times 10^{-1} & 4.22223 \times 10^{-6} & 5.1508 \times 10^{-6} & 7.70341 \times 10^{-4} \\
0 . & 4.22223 \times 10^{-6} & -2.01634 \times 10^{-1} & 1.33333 \times 10^{-5} & 1.9941 \times 10^{-3} \\
0 . & 5.1508 \times 10^{-6} & 1.33333 \times 10^{-5} & -2.01629 \times 10^{-1} & 2.43264 \times 10^{-3} \\
0 . & 7.70341 \times 10^{-4} & 1.9941 \times 10^{-3} & 2.43264 \times 10^{-3} & 1.62175 \times 10^{-1}
\end{array}\right) \\
D_{R}^{e \prime}=\left(\begin{array}{ccccc}
1.62204 \times 10^{-1} & 0 . & 0 . & 0 . & 0 . \\
0 . & 1.62203 \times 10^{-1} & 3.60409 \times 10^{-6} & 4.87783 \times 10^{-4} & -2.59066 \times 10^{-6} \\
0 . & 3.60409 \times 10^{-6} & 1.62184 \times 10^{-1} & -2.68815 \times 10^{-3} & 1.4277 \times 10^{-5} \\
0 . & 4.87783 \times 10^{-4} & -2.68815 \times 10^{-3} & -2.01614 \times 10^{-1} & 1.93227 \times 10^{-3} \\
0 . & -2.59066 \times 10^{-6} & 1.4277 \times 10^{-5} & 1.93227 \times 10^{-3} & 1.62194 \times 10^{-1}
\end{array}\right)
\end{gathered}
$$




\section{APPENDIX E: NUMERICAL COMPARISON FOR THE QUARK SECTOR}

As we did in Appendix D, we carry out the same approach with the most converged numerical point $\left(\chi_{\mathrm{CKM}}^{2}=956.828\right)$ for the up- and down-type quark sectors.

$$
\begin{aligned}
M^{u} & =\left(\begin{array}{ccccc}
0 & 0 & 0 & 0 & 0 \\
0 & 0 & 0 & 0 & 14.474 \\
0 & 0 & 0 & 1206.340 & 277.563 \\
0 & 0 & 273.503 & -1775.200 & 0 \\
0 & 550.990 & 434.462 & 0 & -5624.050
\end{array}\right) \\
M^{d} & =\left(\begin{array}{ccccc}
0 & 0 & 0 & 0 & -0.938 \\
0 & 0 & 0 & 0 & -4.041 \\
0 & 0 & 0 & 1206.340 & -27.427 \\
0 & 0 & -5.636 & -1775.200 & 0 \\
0 & 72.915 & -75.760 & 0 & 2623.620
\end{array}\right)
\end{aligned}
$$

For the comparison, we find the analytic mixing matrices $\left(V_{L, R}^{u, d}\right)_{\text {ana }}$ and the numerical mixing matrices $\left(V_{L, R}^{u, d}\right)_{\text {num }}$ and then subtract one from another after taking absolute value. The numerical differences are given by:

$$
\begin{aligned}
&\left|\left(V_{L}^{u}\right)_{\text {ana }}\right|-\left|\left(V_{L}^{u}\right)_{\text {num }}\right|=\left(\begin{array}{ccccc}
0 & 0 & 0 & 0 & 0 \\
0 & -9.63899 \times 10^{-10} & -8.4547 \times 10^{-6} & 1.12194 \times 10^{-5} & 5.10645 \times 10^{-7} \\
0 & 8.60152 \times 10^{-7} & -4.562 \times 10^{-3} & 6.12434 \times 10^{-3} & -3.20031 \times 10^{-4} \\
0 & -4.32016 \times 10^{-5} & 6.11329 \times 10^{-3} & -4.57314 \times 10^{-3} & 3.70205 \times 10^{-4} \\
0 & -6.95382 \times 10^{-8} & 3.44869 \times 10^{-5} & 4.09197 \times 10^{-5} & -2.39443 \times 10^{-6}
\end{array}\right) \\
&\left|\left(V_{R}^{u}\right)_{\text {ana }}\right|-\left|\left(V_{R}^{u}\right)_{\text {num }}\right|=\left(\begin{array}{ccccc}
0 & 0 & 0 & 0 & 0 \\
0 & -5.36541 \times 10^{-8} & 4.20284 \times 10^{-8} & 3.73725 \times 10^{-8} & 4.77149 \times 10^{-7} \\
0 & -1.56456 \times 10^{-4} & -4.71406 \times 10^{-5} & -7.74521 \times 10^{-4} & 1.34741 \times 10^{-3} \\
0 & 1.27331 \times 10^{-4} & -7.96888 \times 10^{-4} & 6.8947 \times 10^{-5} & 5.76999 \times 10^{-5} \\
0 & 1.86671 \times 10^{-4} & 1.32945 \times 10^{-3} & 2.03335 \times 10^{-5} & -1.39892 \times 10^{-4}
\end{array}\right) \\
&\left|\left(V_{L}^{d}\right)_{\text {ana }}\right|-\left|\left(V_{L}^{d}\right)_{\text {num }}\right|=\left(\begin{array}{cccccc}
7.62437 \times 10^{-9} & 3.28418 \times 10^{-8} & -7.80564 \times 10^{-7} & -5.52416 \times 10^{-7} & 9.20155 \times 10^{-9} \\
-2.16144 \times 10^{-7} & -9.31034 \times 10^{-7} & -2.34437 \times 10^{-6} & 3.16788 \times 10^{-6} & -5.46568 \times 10^{-8} \\
-2.49349 \times 10^{-8} & -1.07406 \times 10^{-7} & 3.14516 \times 10^{-6} & -2.37145 \times 10^{-6} & 9.52623 \times 10^{-8} \\
\left|\left(V_{R}^{d}\right)_{\text {ana }}\right|-\left|\left(V_{R}^{d}\right)_{\text {num }}\right| & =\left(\begin{array}{cccccc}
0 \\
0 & 0 & 3.34474 \times 10^{-10} & 5.01567 \times 10^{-8} & 3.51026 \times 10^{-8} & -1.24719 \times 10^{-9}
\end{array}\right) \\
0 & 6.97453 \times 10^{-6} & -4.09035 \times 10^{-5} & -7.75726 \times 10^{-8} & 1.54578 \times 10^{-6} \\
0 & -4.13664 \times 10^{-5} & 6.52624 \times 10^{-6} & -1.24584 \times 10^{-7} & 1.44401 \times 10^{-5} \\
0 & 6.55855 \times 10^{-8} & -3.67395 \times 10^{-7} & -3.73704 \times 10^{-10} & 6.10094 \times 10^{-8} \\
0 & 2.63961 \times 10^{-6} & 1.5191 \times 10^{-5} & 4.55174 \times 10^{-8} & -5.58627 \times 10^{-7}
\end{array}\right) \\
&(\mathrm{E} 2)
\end{aligned}
$$

Here we can see that the differences for the charged lepton or down-type quark sectors are at most of the order of maximally order of $10^{-5}$, whereas the maximal difference for the up-quark sector goes up to the order of $10^{-3}$ due to the sizeable offdiagonal $\mathcal{O}(1)$ element but it is still a good approximation. Now we confirm that the numerical matrices of $Z$ couplings with quarks $D_{L, R}^{u, d \prime}$ in the mass basis are given by: 


$$
\begin{aligned}
D_{L}^{u \prime}= & \left(\begin{array}{ccccc}
2.55713 \times 10^{-1} & 0 & 0 & 0 & 0 \\
0 & 2.55711 \times 10^{-1} & -3.14988 \times 10^{-5} & 3.18699 \times 10^{-5} & -7.55591 \times 10^{-4} \\
0 & -3.14988 \times 10^{-5} & 2.55083 \times 10^{-1} & 6.37525 \times 10^{-4} & -1.51148 \times 10^{-2} \\
0 & 3.18699 \times 10^{-5} & 6.37525 \times 10^{-4} & 2.55068 \times 10^{-1} & 1.52929 \times 10^{-2} \\
0 & -7.55591 \times 10^{-4} & -1.51148 \times 10^{-2} & 1.52929 \times 10^{-2} & -1.06859 \times 10^{-1}
\end{array}\right) \\
D_{R}^{u \prime}= & \left(\begin{array}{ccccc}
-1.08136 \times 10^{-1} & 0 & 0 & 0 & 0 \\
0 & -1.07941 \times 10^{-1} & -7.52723 \times 10^{-4} & 8.3786 \times 10^{-3} & 1.3608 \times 10^{-4} \\
0 & -7.52723 \times 10^{-4} & -1.05225 \times 10^{-1} & -3.24002 \times 10^{-2} & -5.26224 \times 10^{-4} \\
0 & 8.3786 \times 10^{-3} & -3.24002 \times 10^{-2} & 2.52512 \times 10^{-1} & 5.85742 \times 10^{-3} \\
0 & 1.3608 \times 10^{-4} & -5.26224 \times 10^{-4} & 5.85742 \times 10^{-3} & -1.08041 \times 10^{-1}
\end{array}\right) \\
D_{L}^{d \prime}= & \left(\begin{array}{ccccc}
-3.09781 \times 10^{-1} & 0 & 0 & 0 \\
0 & -3.0978 \times 10^{-1} & 4.35109 \times 10^{-6} & 8.49277 \times 10^{-6} & 4.62349 \times 10^{-4} \\
0 & 4.35109 \times 10^{-6} & -3.09749 \times 10^{-1} & 6.287 \times 10^{-5} & 3.42266 \times 10^{-3} \\
0 & 8.49277 \times 10^{-6} & 6.287 \times 10^{-5} & -3.09658 \times 10^{-1} & 6.68059 \times 10^{-3} \\
0 & 4.62349 \times 10^{-4} & 3.42266 \times 10^{-3} & 6.68059 \times 10^{-3} & 5.39124 \times 10^{-2}
\end{array}\right) \\
D_{R}^{d \prime}= & \left(\begin{array}{ccccc}
5.4068 \times 10^{-2} & 0 & 0 & 0 & 0 \\
0 & 5.40678 \times 10^{-2} & 4.0453 \times 10^{-7} & 2.15284 \times 10^{-4} & -3.09181 \times 10^{-6} \\
0 & 4.0453 \times 10^{-7} & 5.40667 \times 10^{-2} & -6.8355 \times 10^{-4} & 9.81685 \times 10^{-6} \\
0 & 2.15284 \times 10^{-4} & -6.8355 \times 10^{-4} & -3.09705 \times 10^{-1} & 5.22435 \times 10^{-3} \\
0 & -3.09181 \times 10^{-6} & 9.81685 \times 10^{-6} & 5.22435 \times 10^{-3} & 5.39929 \times 10^{-2}
\end{array}\right)
\end{aligned}
$$

where the prefactor $g / c_{w}$ was included in those matrices. The most interesting case of Eq. (E3) is $D_{L}^{d \prime}$ since we know that the left-handed down-type quark sector can access to all mixings among the three SM generations. The used numerical mixing matrix $V_{L}^{d}$ is given by:

$$
V_{L}^{d}=\left(\begin{array}{ccccc}
9.74095 \times 10^{-1} & -2.26141 \times 10^{-1} & 0 & 0 & 0 \\
2.26 \times 10^{-1} & 9.73488 \times 10^{-1} & -2.81626 \times 10^{-2} & -2.12226 \times 10^{-2} & 1.27099 \times 10^{-3} \\
7.97153 \times 10^{-3} & 3.43371 \times 10^{-2} & 7.98097 \times 10^{-1} & 6.01423 \times 10^{-1} & 9.40883 \times 10^{-3} \\
-6.67815 \times 10^{-6} & -2.87659 \times 10^{-5} & 6.01585 \times 10^{-1} & -7.98598 \times 10^{-1} & 1.83648 \times 10^{-2} \\
-3.62201 \times 10^{-4} & -1.56017 \times 10^{-3} & -1.85253 \times 10^{-2} & 9.03631 \times 10^{-3} & 9.99786 \times 10^{-1}
\end{array}\right),
$$

and we can confirm all elements of the first row and column of $D_{L}^{d \prime}$ cancel each other, so identifying the given result in Eq. (E3). What we found in this Appendix verifies the fact that the SM $Z$ physics does not get affected by any specific choice of basis.

[1] G. Aad et al. (ATLAS Collaboration), Phys. Lett. B 716, 1 (2012).

[2] S. Chatrchyan et al. (CMS Collaboration), Phys. Lett. B 716, 30 (2012).

[3] L. Calibbi and G. Signorelli, Riv. Nuovo Cimento 41, 71 (2018).

[4] A. E. C. Hernández, S. F. King, and H. Lee, Phys. Rev. D 103, 115024 (2021).
[5] J. Hernandez-Garcia and S. F. King, J. High Energy Phys. 05 (2019) 169.

[6] S. F. King, J. High Energy Phys. 09 (2018) 069.

[7] A. E. C. Hernández, S. F. King, H. Lee, and S. J. Rowley, Phys. Rev. D 101, 115016 (2020).

[8] S. Raby and A. Trautner, Phys. Rev. D 97, 095006 (2018).

[9] M. Lindner, M. Platscher, and F. S. Queiroz, Phys. Rep. 731, 1 (2018). 
[10] L. Lavoura, Eur. Phys. J. C 29, 191 (2003).

[11] C. W. Chiang, Y. F. Lin, and J. Tandean, J. High Energy Phys. 11 (2011) 083.

[12] A. Crivellin, F. Kirk, C. A. Manzari, and M. Montull, J. High Energy Phys. 12 (2020) 166.

[13] A. M. Baldini et al. (MEG Collaboration), Eur. Phys. J. C 76, 434 (2016).

[14] B. Aubert et al. (BaBar Collaboration), Phys. Rev. Lett. 104, 021802 (2010).

[15] K. Hayasaka et al., Phys. Lett. B 687, 139 (2010).

[16] P. Abreu et al. (DELPHI Collaboration), Z. Phys. C 73, 243 (1997).

[17] F. Z. Xu, W. Zhang, J. Li, and T. Li, Phys. Rev. D 98, 115033 (2018).

[18] G. Guedes and J. Santiago, arXiv:2107.03429.

[19] P. N. Bhattiprolu and S. P. Martin, Phys. Rev. D 100, 015033 (2019).
[20] A. M. Sirunyan et al. (CMS Collaboration), Phys. Rev. D 100, 052003 (2019).

[21] V. De Romeri, M. J. Herrero, X. Marcano, and F. Scarcella, Phys. Rev. D 95, 075028 (2017).

[22] L. Calibbi, X. Marcano, and J. Roy, Eur. Phys. J. C 81, 1054 (2021).

[23] M. Aaboud et al. (ATLAS Collaboration), J. High Energy Phys. 07 (2018) 176.

[24] G. C. Branco, J. T. Penedo, P. M. F. Pereira, M. N. Rebelo, and J. I. Silva-Marcos, J. High Energy Phys. 07 (2021) 099.

[25] P. A. Zyla et al. (Particle Data Group), Prog. Theor. Exp. Phys. 2020, 083C01 (2020).

[26] B. Belfatto and Z. Berezhiani, J. High Energy Phys. 10 (2021) 079.

[27] B. Belfatto, R. Beradze, and Z. Berezhiani, Eur. Phys. J. C 80, 149 (2020).

[28] S. F. King, J. High Energy Phys. 09 (2002) 011. 